\title{
OSCILLATION THEOREMS FOR PRIMES IN ARITHMETIC PROGRESSIONS AND FOR SIFTING FUNCTIONS
}

\author{
JOHN FRIEDLANDER, ANDREW GRANVILLE, \\ ADOLF HILDEBRAND, AND HELMUT MAIER
}

\section{CONTENTS}

I. Introduction

1. Primes in arithmetic progressions

2. The main theorems

II. Oscillation theorems for sifting functions

3. The statements

4. Proof of Theorem B2

5. Proof of Proposition 3.1

6. Proof of Proposition 3.2

7. Proof of Theorem C: preliminaries

8. Proof of Theorem C: completion

9. Proof of Theorem B1

10. Proof of Theorem B3

III. Oscillation theorems for primes

11. Proof of Theorem A1

12. Proof of Theorem A2

13. Proofs of Theorem A3 and Proposition 2.1

\section{INTRODUCTION}

\section{PRIMES IN ARITHMETIC PROGRESSIONS}

For $x \geq 2$ real, $q$ a positive integer, and $a$ an integer coprime to $q$, let $\theta$ and $\Delta$ be defined by

$$
\theta(x ; q, a)=\sum_{\substack{p \leq x \\ p \equiv a \bmod q}} \log p=\frac{x}{\varphi(q)}(1+\Delta(x ; q, a)),
$$

Received by the editors June 15, 1989.

1980 Mathematics Subject Classification (1985 Revision). Primary 11N13; Secondary 11N25, $11 \mathrm{~N} 35$.

The first two authors were partially supported by NSERC and the last two authors were partially supported by NSF. 
where $\varphi$ is Euler's function. The prime number theorem for arithmetic progressions is equivalent to the statement that $\Delta(x ; q, a)=o(1)$ holds, as $x \rightarrow \infty$, for fixed $q$ and $a$. The function $\Delta$ thus provides a measure for the regularity of distribution of primes in arithmetic progressions.

The main focus in the study of primes in arithmetic progressions has previously been on estimating $\Delta(x ; q, a)$ from above uniformly for all, or almost all, $q \leq Q$, with $Q$ as large as possible. The two fundamental theorems in this connection (cf. [Da]) are the Siegel-Walfisz and the Bombieri-Vinogradov theorems. It follows from the former that the bound:

$$
|\Delta(x ; q, a)| \ll(\log x)^{-A}
$$

holds uniformly for $q \leq(\log x)^{B}$, for any fixed positive constants $A$ and $B$. The latter states, in one form, that

$$
\sum_{q \leq Q} \frac{1}{\varphi(q)} \max _{(a, q)=1}|\Delta(x ; q, a)| \ll(\log x)^{-A}
$$

holds for any $A>0$ with $Q=\sqrt{x}(\log x)^{-B}$, where $B$ is a suitable constant depending on $A$. As a consequence, the estimate (1.2) is true for all but $o(Q)$ moduli $q \leq Q$, provided that $Q \leq \sqrt{x}(\log x)^{-C}$ with a sufficiently large constant $C$.

It is generally expected that these results are far from being the best possible. In fact, under the Generalized Riemann Hypothesis, (1.2) is known to hold for all $q \leq \sqrt{x}(\log x)^{-B}$ and all $a$ coprime with $q$, if $B$ is a sufficiently large constant. However, even this conditional result probably is not optimal, and stronger conjectures about the size of $\Delta(x ; q, a)$ and the range of validity of estimates like (1.2) or (1.3) have been put forward.

Perhaps the best known of these conjectures is the Elliott-Halberstam conjecture $[\mathrm{EH}]$, according to which the Bombieri-Vinogradov estimate is valid for $Q=x^{1-\varepsilon}$ and possibly even for $Q=x(\log x)^{-B}$ with a sufficiently large constant $B$; see [EH, p. 62] for the precise formulation. This conjecture was at least partially motivated by probabilistic considerations; in fact, Elliott [E1] had shown earlier that, in an appropriate sense, "almost all" primelike sequences of positive integers satisfy a Bombieri-Vinogradov type estimate (1.3) with $Q=x(\log x)^{-B}, B=B(A)$.

A corresponding conjecture for the estimate of individual terms $\Delta(x ; q, a)$ has been made by Montgomery [Mo]. It implies that

$$
|\Delta(x ; q, a)| \ll_{\varepsilon}(q / x)^{1 / 2-\varepsilon} \log x
$$

holds uniformly for $q \leq x$, for any given $\varepsilon>0$. A weaker form of (1.4) may 
be given as

$$
|\Delta(x ; q, a)| \ll(q / x)^{1 / 2} x^{\varepsilon}
$$

The latter estimate is only of interest for $q \leq x^{1-\varepsilon}$, while (1.4) remains nontrivial for moduli as large as $x$ divided by a fixed power of $\log x$.

The strongest forms of both of these conjectures were recently disproved by Friedlander and Granville [FG]. They showed that for any $A>0$ there exist arbitrarily large values of $x$ and integers $q \leq x(\log x)^{-A}$ and $(a, q)=1$, for which $|\Delta(x ; q, a)| \gg 1$, thus disproving Montgomery's conjecture (1.4). Moreover, such lower bounds hold for most moduli $q$, so that (1.2) even fails when averaged over a suitable range for $q$. As a consequence, the BombieriVinogradov type estimate (1.3) cannot be valid with $Q$ as large as $x$ divided by any fixed power of $\log x$.

The main purpose of this paper is to extend these results by showing that the mentioned conjectures already fail for moduli that are significantly smaller than $x$ divided by any fixed power of $\log x$. Specifically, we shall prove that (1.4) fails to hold for all moduli $q$ as small as $x \exp \left(-(\log x)^{1 / 5-\delta}\right)$, most moduli $q$ as small as $x \exp \left(-(\log x)^{1 / 3-\delta}\right)$, and many moduli $q$ as small as $x \exp \left(-(\log x)^{1 / 2-\delta}\right)$, for any fixed $\delta>0$, if the parameter $\varepsilon$ in (1.4) is sufficiently small. We also show that, given any positive constant $A$, the estimate (1.3) is false for all sufficiently large $x$ with $Q=x \exp \left\{-(1-\varepsilon) A\left(\log _{2} x\right)^{2} / \log _{3} x\right\}$, where throughout, $\log _{k} x$ denotes the $k$ times iterated logarithm.

We state our main results in three theorems, Theorems A1-A3, which are proven by rather different methods. The first method is closest in spirit to the argument of Friedlander and Granville [FG]. It has its roots in a paper of Maier [Ma], in which he showed that the asymptotic formula $\pi(x+y)-\pi(x) \sim y / \log x$ fails to hold when $y=(\log x)^{\lambda}$, for any fixed positive $\lambda$. The second method is a modification of the first, while the third is new and quite different from the other two approaches. These ideas are briefly discussed following the statements.

Common to all three methods is the fact that they ultimately depend on oscillation results for certain sifting functions, a typical example being the wellknown function:

$$
\Phi(y, z)=\#\{n \leq y: p \mid n \Rightarrow p \geq z\} .
$$

Such oscillation results turn out to be quite difficult to prove. While it is relatively easy to obtain upper bounds for the oscillations of functions like $\Phi(y, z)$, thereby showing that these functions behave in a regular manner to some extent, it is much harder to exhibit irregularities of such functions. In Chapter II we prove three such oscillation theorems, Theorems B1-B3, which are applied to prove Theorems A1-A3, respectively in Chapter III. Two of these theorems depend on a further and more fundamental result, Theorem $\mathrm{C}$, which gives an "asymptotic formula" for the amount of oscillations of the function $\Phi(y, z)$. This theorem is quite difficult to prove and takes up the bulk of Chapter II. 


\section{THE MAIN THEOREMS}

We consider here only the error function $\Delta(x ; q, a)$ defined in (1.1). It is perhaps more customary to deal with the functions $\tilde{\Delta}, \bar{\Delta}$ defined by

$$
\pi(x ; q, a)=\sum_{\substack{p \leq x \\ p \equiv a \bmod q}} 1=\frac{\operatorname{li} x}{\varphi(q)}(1+\tilde{\Delta}(x ; q, a))
$$

and

$$
\psi(x ; q, a)=\sum_{\substack{n \leq x \\ n \equiv a \bmod q}} \Lambda(n)=\frac{x}{\varphi(q)}(1+\bar{\Delta}(x ; q, a)) .
$$

In fact, although we find $\Delta$ slightly simpler to work with, similar results hold for $\tilde{\Delta}, \bar{\Delta}$.

Our first theorem gives lower bounds for the oscillations of the function $\Delta(x ; q, a)$ in the case of moduli $q$ that do not have too many small prime factors.

Theorem A1. Let $\varepsilon>0$. Then for all $q \geq q_{0}(\varepsilon)$ and all $x$ satisfying

$$
q(\log q)^{1+\varepsilon}<x \leq q \exp \left((\log q)^{5 / 11-\varepsilon}\right),
$$

there exist numbers $x_{ \pm}$with $x / 2<x_{ \pm} \leq 2 x$ and integers $a_{ \pm}$coprime with $q$ such that

$$
\begin{gathered}
\Delta\left(x_{+} ; q, a_{+}\right) \geq y^{-(1+\varepsilon) \delta_{1}(x, y)}, \\
\Delta\left(x_{-} ; q, a_{-}\right) \leq-y^{-(1+\varepsilon) \delta_{1}(x, y)},
\end{gathered}
$$

where $y=x / q$ and $\delta_{1}(x, y)=\log (\log y / \log 2 x) / \log (\log x / \log y)$.

The right-hand sides of (2.3) and (2.4) are $\gg 1$ if $y=(\log x)^{O(1)}$, and are $\gg y^{-(1+\varepsilon) \alpha /(1-\alpha)}$ if $y=\exp \left((\log x)^{\alpha}\right)$ with $0<\alpha \leq 1 / 3$. Thus the theorem shows that Montgomery's conjecture (1.4) is incorrect for moduli $q$ as small as $x \exp \left(-(\log x)^{1 / 3-\delta}\right)$, for any given positive $\delta$.

The lower bounds in (2.3) and (2.4) as well as the range (2.2) for $x$ are essentially the limits of what can be achieved by our method of proof. In connection with the lower bound in (2.2) it should be pointed out that for $x<(1-\varepsilon) \varphi(q) \log q$, large lower bounds for $\Delta$ follow trivially from the fact that there are more reduced classes modulo $q$ than there are primes $\leq x$. As far as the upper bound is concerned, we note that the theorem is not of much interest in the range $q<x \exp \left(-(\log x)^{1 / 3+\varepsilon}\right)$, since then $\delta_{1}(x, y)>1 / 2$ and the lower bounds in (2.3) and (2.4) become smaller than the bounds predicted 
by conjectures like (1.4). Under the assumption of the Generalized Riemann Hypothesis, the exponent $\delta_{1}(x, y)$ can be reduced to $\log \left(\log y / \log _{2} x\right) / \log _{2} x$.

Theorem A1 sharpens and extends to a much larger range the corresponding result of Friedlander and Granville [FG], and represents the analogue for arithmetic progressions of a recent result of Hildebrand and Maier [HM] on irregularities in the distribution of primes in short intervals. This latter result asserts that bounds of the type (2.3) and (2.4) hold with the same function $\delta_{1}$ for all sufficiently large $x$ and $2 \leq y \leq \exp \left(A(\log x)^{1 / 3}\right)$, if $\Delta\left(x_{ \pm} ; q, a_{ \pm}\right)$is replaced by $\Delta\left(x_{ \pm}, y\right)$ for suitable $x_{ \pm}, x \leq x_{ \pm} \leq 2 x$, where

$$
\Delta(x, y)=\frac{1}{y} \sum_{x<p \leq x+y} \log p-1
$$

and $A$ is a positive constant.

The proof of Theorem Al combines the methods of [FG] and [HM]. The basic idea, which is essentially due to Maier [Ma], is to consider the occurrence of primes in a rectangular array $r P+q s$, where $r$ and $s$ run independently through the integers in certain intervals. Here $q$ is the modulus of the progression in which we are interested. Thus, for each fixed $r$ and varying $s$, we are considering the primes in a progression modulo $q$ and trying to prove that this number is not always close to what we "expect." On the other hand, for each fixed $s$ and varying $r$, we are considering the primes in an arithmetic progression modulo $P$. Here $P$ is chosen much smaller than $q$ so that the number of such primes can be accurately estimated. This estimate, which rests on the Linnik-Gallagher prime number theorem, then reduces the problem to counting the number of $s$ satisfying $(s, P)=1$. Now, if this number is more (or less) than the expected number, then there will be correspondingly more (or less) primes than expected in some progression modulo $q$.

Thus one is led to study oscillations of sifting functions of the type $\#\{s \leq y:(s, P)=1\}$. In the case where $P$ is of the form $P=\prod_{p<z} p$, these functions reduce to the well-known function $\Phi(y, z)$. Oscillation theorems for $\Phi(y, z)$ have been given in [Ma] (see also [Iw, p. 211]) for the range $\log y \ll \log z$, and in [HM] for the range $\log y \ll z^{1 / 2-\varepsilon}$. These results would not be sufficient for the proof of Theorem A1, however, and therefore, we prove a new oscillation result, Theorem B1, which is much sharper than the previous results.

The above argument requires that $(q, P)=1$ and thus, when $P$ is chosen as the product of all primes $<z$, it applies only to moduli $q$ having no prime factors $<z$. For more general $q$ it is necessary to modify the choice of $P$ and, hence, also the definition of $\Phi(y, z)$, to exclude the small prime factors of $q$. Provided $q$ does not have too many small prime divisors, the modified function can be shown to exhibit essentially the same oscillatory behavior. This is the genesis of condition (2.1). It is possible to deduce from the Turán-Kubilius inequality that almost all integers $q$ have $(1+o(1)) \sum_{p<\log q}(1 / p)=(1+o(1)) \log _{3} q$ 
distinct prime factors less than $\log q$ and so almost all integers satisfy (2.1) (see, e.g., [E2, Chapter 4]).

Condition (2.1) could probably be relaxed to some extent, but in this approach it cannot be completely removed without sacrificing the quality of the result. Our next theorem gives a result that is somewhat weaker than the estimates of Theorem A1, but has the advantage of being valid for all $q$.

Theorem A2. Let $\varepsilon>0$. There exist $N(\varepsilon)>0$ and $q_{0}=q_{0}(\varepsilon)>0$ such that for any $q>q_{0}$ and any $x$ with

$$
q(\log q)^{N(\varepsilon)}<x \leq q \exp \left((\log q)^{1 / 3}\right),
$$

there exist numbers $x_{ \pm}$with $x / 2<x_{ \pm} \leq 2 x$ and integers $a_{ \pm}$coprime with $q$ such that

$$
\Delta\left(x_{+} ; q, a_{+}\right) \geq \frac{1}{\log ^{5} x} y^{-(1+\varepsilon) \delta_{2}(x, y)},
$$

$$
\Delta\left(x_{-} ; q, a_{-}\right) \leq-\frac{1}{\log ^{5} x} y^{-(1+\varepsilon) \delta_{2}(x, y)},
$$

where $y=x / q$ and $\delta_{2}(x, y)=3 \log \left(\log y / \log _{2} x\right) / \log (\log x \log y)$.

This result is obtained by taking $P$ in the above argument to be a product of primes chosen from an appropriate short interval. One consequence of this is that we are able to exclude all prime factors of $q$ even if there are many of these. Another is that the close proximity of all prime factors of $P$ allows us to deduce the oscillation result by combinatorial means.

The factor $1 / \log ^{5} x$ is significant only if $\log y \ll\left(\log _{2} x\right)^{2}$. If two-sided estimates are not required and only a lower bound for $|\Delta|$ is sought, then this factor may be replaced by $\log y /\left(\log _{2} x\right)^{2}$; see the remarks after the proof.

As compared with Theorem A1, the exponent $\delta_{2}$ is somewhat worse than $\delta_{1}$, being less than $\frac{1}{2}$ only for $y<\exp \left(\log ^{1 / 5-\varepsilon} x\right)$. (Assuming the Generalized Riemann Hypothesis this too may be improved.) However, Theorem A2 has the advantage of being valid for all $q$ and has the additional advantage that the required oscillation result, Theorem B2 (see $\S \S 3,4)$, is much easier to prove.

Next we turn to lower bounds for averages over $\Delta(x ; q, a)$. As almost all $q$ satisfy the hypothesis of Theorem A1, we immediately obtain from Theorem A1 the following result:

Corollary 2.1. For all sufficiently large $x$ and $2 x \exp \left(-(\log x)^{5 / 11-\varepsilon}\right) \leq Q \leq$ $x / 2$, we have

$$
\sum_{q \leq Q} \frac{1}{\varphi(q)} \max _{x^{\prime} \leq x} \max _{(a, q)=1}\left|\Delta\left(x^{\prime} ; q, a\right)\right| \gg y^{-(1+\varepsilon) \delta_{1}(x, y)},
$$

where $y=x / Q$. 
Theorem A2 yields a similar, but slightly weaker result. Stronger results may be obtained by bounding from below averages of the type

$$
\sum_{\substack{q<Q \\(q, a)=1}} \frac{1}{\varphi(q)}|\Delta(x ; q, a)|=\frac{1}{x} \sum_{\substack{q<Q \\(q, a)=1}}\left|\theta(x ; q, a)-\frac{x}{\varphi(q)}\right|
$$

or

$$
\sum_{\substack{q \sim Q \\(q, a)=1}} \frac{1}{\varphi(q)} \Delta(x ; q, a)=\frac{1}{x} \sum_{\substack{q \sim Q \\(q, a)=1}}\left(\theta(x ; q, a)-\frac{x}{\varphi(q)}\right),
$$

where $a$ is a fixed nonzero integer and $q \sim Q$ means $Q<q \leq 2 Q$. Such averages have been considered in recent work of Fouvry [Fo], and Bombieri, Friedlander, and Iwaniec [BFI], where nontrivial upper bounds have been given for (2.9) and (2.10) in ranges beyond the known range of validity of the BombieriVinogradov theorem in its original form (1.3). Thus, it is of interest to obtain lower bounds for these quantities similar to the bound (2.8), and, of course, such bounds provide, a fortiori, a lower bound for the left-hand side of $(2.8)$. Our third theorem gives such a result.

Theorem A3. Let $\varepsilon>0$. Then for some $c>0$, for all $x>x_{0}(\varepsilon)$, and all $Q$ satisfying

$$
x \exp (-c \sqrt{\log x})<Q \leq x(\log x)^{-(1+\varepsilon)},
$$

there exist $Q_{ \pm}$with $Q / 2<Q_{ \pm} \leq 2 Q$ and integers $a_{ \pm}$such that

$$
\sum_{\substack{q \sim Q_{+} \\\left(q, a_{+}\right)=1}} \frac{1}{\varphi(q)} \Delta\left(x ; q, a_{+}\right) \geq \frac{1}{\log _{2} x} y^{-(1+\varepsilon) \delta_{3}(x, y)},
$$

$$
\sum_{\substack{q \sim Q_{-} \\\left(q, a_{-}\right)=1}} \frac{1}{\varphi(q)} \Delta\left(x ; q, a_{-}\right) \leq-\frac{1}{\log _{2} x} y^{-(1+\varepsilon) \delta_{3}(x, y)},
$$

where $y=x / Q$ and $\delta_{3}(x, y)=\log \left(\log y / \log _{2} x\right) / \log _{2} x$.

We remark that $\delta_{3}$ is smaller, and hence better, than the exponents $\delta_{1}$ and $\delta_{2}$ from Theorems $\mathrm{A} 1$ and $\mathrm{A} 2$, and indeed is the same exponent achieved by the method of Theorem A1 on the assumption of the Generalized Riemann Hypothesis. The result remains of interest in the entire range $y<\exp \left((\log x)^{1 / 2-\varepsilon}\right)$ and, with more effort, could be somewhat sharpened so that the bound remained larger than $y^{-1 / 2}$ in the range $y<\exp (c \sqrt{\log x})$.

The factor $1 / \log _{2} x$ in (2.12) and (2.13) is significant only for $\log y \ll$ $\log _{2} x \log _{3} x$. In fact, for this latter range it can also be shown that this factor may be dropped, if one uses the method that gives Theorem A1, provided that the sum $\sum_{q \sim Q,\left(q, a_{ \pm}\right)=1}$ is replaced by the sum $\sum_{q \sim Q} \max _{(a, q)=1}$ (respectively $\left.\sum_{q \sim Q} \min _{(a, q)=1}\right)$. 
Since $y^{\delta_{3}(x, y)}=(\log x)^{(1+o(1)) A}$ if $\log y \sim A\left(\log _{2} x\right)^{2} / \log _{3} x \quad(A>0$ fixed $)$, we deduce from Theorem $A 3$ the following result, which sets a limit on the range of $Q$ for which Bombieri-Vinogradov type estimates for averages of the form (2.9) may hold.

Corollary 2.2. Let $\varepsilon>0, A>0$. For every $x>x_{0}(\varepsilon, A)$, there exists an integer a such that for all $Q$ with

$$
Q \geq x \exp \left(-(1-\varepsilon) A\left(\log _{2} x\right)^{2} / \log _{3} x\right)
$$

we have

$$
\sum_{\substack{q<Q \\(q, a)=1}}\left|\theta(x ; q, a)-\frac{x}{\varphi(q)}\right| \geq\left|\sum_{\substack{q \sim Q / 2 \\(q, a)=1}}\left(\theta(x ; q, a)-\frac{x}{\varphi(q)}\right)\right|>x(\log x)^{-A} .
$$

The method of proof of Theorem A3 is rather different from that of Theorems A1, A2. It is based on the following simple idea. We consider primes $p \sim x$, and for given $a$ we wish to study $p \equiv a(\bmod q)$ on average over $q$. This leads to a double sum over $p$ and $q$. If we write $p-a=q r$, then the double sum can be transformed to a double sum over $p$ and $r$.

Since our values for $q$ are almost as large as $x$, the complementary factor $r$ is quite small, and the transformed sum deals with primes in progressions where the modulus $r$ is sufficiently small so that analytic methods can be successfully applied. The resulting estimates lead to a sum that, in the special case that $a$ is of the form $\prod_{p<z} p$, can be shown to have an oscillatory behavior, which leads to the conclusion in a manner similar to those of the above methods.

In contrast to the earlier methods, this one offers the advantages of leading to a stronger result (at least when $x / q$ is not too small), and of not requiring the rather deep Linnik-Gallagher theorem for its proof. This method seems to be incapable of dealing with individual arithmetic progressions, however, and thus cannot even give, as far as one can see, a new proof of Maier's original result [Ma].

In the case where $Q>x(\log x)^{-N}$ for some fixed $N$, Theorem A3 and the corollary recover Theorem 1 of [FG]. As in [FG] we deduce lower bounds for sums $\sum_{q} \mid$ error $\mid$, by bounding from below the smaller quantity $\mid \sum_{q}$ error $\mid$. The integers $a$ produced by the proof grow as $x$ does. We believe that this is inevitable, at least for bounding $\mid \sum_{q}$ error $\mid$. That this is true, at least for $Q$ in the range (2.14), is seen from the following result that slightly refines earlier theorems of Bombieri-Friedlander-Iwaniec [BFI] and Fouvry [Fo]. The proofs of these results are based on the same simple switching principle described in connection with the proof of Theorem A3. 
Proposition 2.1. Fix $A>0$. Then for $2 \leq Q \leq \frac{1}{2} x, 0<|a|<(\log x)^{A}$, we have (2.16)

$$
\left|\sum_{\substack{q \sim Q \\(q, a)=1}}\left(\theta(x ; q, a)-\frac{x}{\varphi(q)}\right)\right| \ll_{A} \tau(a) Q \log (x / Q)+x(\log x)^{-A}+Q \log |a| .
$$

Here the last term on the right may be deleted unless $a$ is prime.

Thus, when $Q$ satisfies both (2.11) and (2.14), (2.12) and (2.13) cannot hold with numbers $a_{ \pm}$in the range $0<\left|a_{ \pm}\right|<(\log x)^{A}$.

We conclude this section with some speculation as to what might be the right upper bound for $\Delta(x ; q, a)$ and formulate the following:

Hypothesis. Let $\varepsilon>0$. For all $q, a$, and $x$ with $q \geq q_{0}(\varepsilon),(a, q)=1$, $x \geq q(\log q)^{1+\varepsilon}$, we have

$$
|\Delta(x ; q, a)| \ll y^{-(1-\varepsilon) \delta(x, y)},
$$

where $y=x / q$ and

$$
\delta(x, y)=\min \left(\frac{1}{2}, \frac{\log \left(\log y / \log _{2} x\right)}{\log _{2} x}\right)=\min \left(\frac{1}{2}, \delta_{3}(x, y)\right) .
$$

It is equally reasonable to expect that the upper bound given by (2.17) and (2.18) holds for the short interval analogue where $\Delta(x ; q, a)$ is replaced by $\Delta(x, y)=y^{-1}(\theta(x+y)-\theta(x)-y)$. These hypotheses carry the implication that the results we obtain on average over $q$ (Theorem A3) and, if GRH be assumed, the results we obtain here for individual progressions and those given for short intervals in $[\mathrm{HM}]$, are essentially best possible.

As already indicated, the proof of Theorem $\mathrm{A} j(1 \leq j \leq 3)$ is dependent upon a Theorem $\mathrm{B} j$, which demonstrates an oscillatory behavior for a certain sifting function of the type $\sum_{n \leq y,(n, P)=1} f(n)$. Those theorems are stated and proven in Chapter II. The proofs of Theorems B1 and B3 depend on a further Theorem $C$ whose proof occupies the greater part of this paper. The final Chapter III gives the derivation of the above-stated results.

\section{OSCILLATION THEOREMS FOR SIFTING FUNCTIONS}

\section{THE STATEMENTS}

In this section we state our principal results on oscillations of $\Phi(y, z)$ and certain related functions. These results are proven in $\S \S 4-10$.

We first introduce some notation. For real numbers $y>0$ and $z \geq 2$ and positive integers $k$, we set

$$
\begin{aligned}
\Phi_{k}(y, z) & =\#\{n \leq y: p \mid n, p \nmid k \Rightarrow p \geq z\} \\
& =y \prod_{\substack{p<z \\
p \nmid k}}\left(1-\frac{1}{p}\right)\left(1+r_{k}(y, z)\right),
\end{aligned}
$$


and let $\Phi(y, z)=\Phi_{1}(y, z), r(y, z)=r_{1}(y, z)$. We further define a weighted form $\bar{r}^{(p)}(y, z)$ of $r$ by

$$
\bar{r}^{(p)}(y, z)=\int_{y / 2}^{y} r^{(p)}(t, z) \frac{d t}{t}
$$

where $r^{(p)}(y, z)$ is defined by

$$
\sum_{\substack{n \leq y \\ p^{\prime} \mid n \Rightarrow p^{\prime} \geq z, p^{\prime} \neq p}} \frac{n}{\phi(n)}=y C \prod_{\substack{p^{\prime}<z \\ \text { or } p^{\prime}=p}}\left(1-\frac{1}{p^{\prime}}\right)\left(1+r^{(p)}(y, z)\right),
$$

with

$$
C=\prod_{\substack{p^{\prime} \geq z \\ p^{\prime} \neq p}}\left(1+\frac{1}{p^{\prime}\left(p^{\prime}-1\right)}\right),
$$

and we set $\bar{r}(y, z)=\bar{r}^{(2)}(y, z)$.

Throughout this and the following sections we work with pairs of real numbers $(y, z)$ lying in a range of the type

$$
\left(R_{\alpha}\right) \quad z \geq z_{0}, \quad y=z^{u}, \quad u_{0} \leq u \leq z^{1-\alpha} \text {, }
$$

where $\alpha$ is a given positive real number, and $z_{0}$ and $u_{0}$ are suitable sufficiently large constants, possibly depending on $\alpha$. Given $y$ and $z$, we define $u$ and $\mathscr{L}$ by

$$
u=\log y / \log z, \quad \mathscr{L}=\log z .
$$

For $u>0$ we define $\xi_{u}$ as the positive solution of the equation:

$$
e^{\xi_{u}}=u \xi_{u}+1
$$

It is easy to see that $\xi_{u}$ satisfies

$$
\begin{gathered}
\xi_{u}=\log u+\log _{2} u+O\left(\frac{\log _{2} u}{\log u}\right) \quad(u \geq 3), \\
\frac{d}{d u} \xi_{u}=\frac{1}{u}+O\left(\frac{1}{u \log u}\right) \quad(u \geq 3)
\end{gathered}
$$

(cf. [HT, Lemma 1]).

We can now state the two oscillation theorems for the functions $r_{k}$ and $\bar{r}^{(p)}$ that are required for the proof of Theorems A1 and A3.

Theorem B1. Let $\varepsilon>0$ and $(y, z)$ be in the range $\left(R_{1 / 6+\varepsilon}\right)$ with $u_{0}=1+\varepsilon$, and let $u=\log y / \log z$. There exist numbers $z_{ \pm}$satisfying

$$
\min \left(\frac{1}{2} z, z^{1-2 /(u+2)}\right) \leq z_{ \pm} \leq z,
$$

such that for every positive integer $k$ satisfying

$$
\sum_{\substack{p \mid k \\ p \leq z}} 1 \leq 2 \log _{2} z
$$


and for some $y_{ \pm}$(that may depend on $k$ ) satisfying

$$
y(1-1 / \log y) \leq y_{ \pm} \leq y
$$

there hold

$$
\left\{\begin{array}{l}
r_{k}\left(y_{+}, z_{+}\right) \geq \exp \left\{-u \xi_{u}-c_{1} u\right\} \\
r_{k}\left(y_{-}, z_{-}\right) \leq-\exp \left\{-u \xi_{u}-c_{1} u\right\}
\end{array}\right.
$$

where $c_{1}$ is a positive constant depending at most on $\varepsilon$.

Theorem B3. Let $\varepsilon>0$ and $(y, z)$ be in $\left(R_{1 / 6+\varepsilon}\right)$ with $u_{0}=1+\varepsilon$. There exist $z_{ \pm}$satisfying (3.4) such that for every prime $p$ and for some $y_{ \pm}$(which may depend on $p$ ) satisfying (3.6) the inequalities (3.7) hold with $r_{k}$ replaced by $\bar{r}^{(p)}$.

In what follows we are primarily concerned with the condition $u>u_{0}$ for a large unspecified $u_{0}$. How this assumption is relaxed to $u \geq 1+\varepsilon$ is indicated at the end of this section. Actually, this weaker condition is necessary for the proofs of Theorems A1 and A3 in that $\log ^{1+\varepsilon}$ in (2.3) and (2.12) would otherwise have to be replaced by $\log ^{u_{0}+\varepsilon}$.

The above results are quite sharp. It can be shown that, in the range $\left(R_{\varepsilon}\right)$, $r_{k}(y, z)$ is bounded from above by $O\left(\exp \left(-u \xi_{u}+O(u)\right)\right)$ (cf. [HM, Lemma 2] for the case $k=1$ ). Thus, the lower bound (3.7) is best possible aside from the value of the constant $c_{1}$. Moreover, the localization of $z_{ \pm}$cannot be much improved. Indeed, it is clear from Theorem $C$ and Proposition 3.2 that the numbers $u_{ \pm}=\log y / \log z_{ \pm}$cannot be better localized than within an interval of length 1 . On the other hand, the localization of $y_{ \pm}$is probably not best possible. In fact, under the Riemann Hypothesis, (3.7) can be shown to hold in the range $\left(R_{1 / 2+\varepsilon}\right)$ for any $\varepsilon>0$ with $y_{ \pm}=y$ (cf. the remarks following Corollary 3.1). For our applications, however, this deficiency is inconsequential.

We remark that, instead of keeping $y$ (essentially) fixed and varying $z$ in order to obtain extreme values of $r_{k}(y, z)$ or $\bar{r}^{(p)}(y, z)$, we could have instead fixed $z$ and varied $y$. Specifically, under the conditions of Theorem B1, it can be shown that the estimate (3.7) holds with $z_{ \pm}=z$ and suitable numbers $y_{ \pm}$satisfying $y \leq y_{ \pm} \leq y z^{2}$. A similar estimate, but with a much weaker localization of $y_{ \pm}$, was obtained in [HM] for the case of $r(y, z)$. This was achieved by bounding from below certain weighted averages over the function $f(u)=\left|f\left(z^{u}, z\right)\right|^{2}$. This method, while being relatively simple, is not capable of yielding results of the quality of Theorems B1 or B3, which will be needed for the proofs of Theorems A1 and A3. Therefore it is necessary to use a different and more complicated approach.

The restriction (3.5) in Theorem B1 is responsible for the corresponding restriction (2.1) in Theorem A1. In order to deal with moduli $k$ containing many small prime factors, it is natural to consider the analogue to $\Phi(y, z)$ obtained by counting all those $n \leq y$ whose prime factors lie in a given interval. In general, this should be more difficult to estimate but, in the case that the 
interval is rather short, a fairly precise oscillation theorem may be obtained by combinatorial means. The following such result is applied to Theorem A2.

Theorem B2. For $z \geq z_{0}, h \leq \frac{1}{2} z, k \geq 1, P$ the product of any $k$ primes, all of which are in the interval $(z-h, z]$, we have

$$
(-1)^{j-1} r_{P}(y) \doteq(-1)^{j-1}\left(\sum_{\substack{n \leq y \\
(n, P)=1}} 1-\frac{\varphi(P)}{P} y\right) \geq \frac{1}{4} y\left(\begin{array}{l}
k \\
j
\end{array}\right) z^{-j}
$$

for every integer $j$ with $1 \leq j \leq k / 5$ and every real $y$ with $(z-h)^{j} \geq y \geq$ $4 j z^{j} /(k-j+1)$.

We deduce Theorems B1 and B3 from a more fundamental result, Theorem $\mathrm{C}$, and two auxiliary propositions. Theorem $\mathrm{C}$ gives an asymptotic formula for a "smoothed" version of $r(y, z)$ in terms of the generating Dirichlet series:

$$
F(s)=F(z ; s)=\prod_{p<z}\left(1-\frac{1}{p^{s}}\right) \zeta(s) .
$$

To state this result, we require some notation. With $F(s)$ defined above, we let

$$
\varphi(s)=\varphi(z ; s)=\log F(z ; s),
$$

where the branch of the logarithm is chosen such that $\varphi(\sigma)$ is real for $0<\sigma<$ 1. Such a branch exists and is an analytic function in the region $0<\sigma<1$, $|t| \leq 1$, since $\zeta(s)$, and hence $F(s)$, is analytic and nonzero in this region. Next, given $\varepsilon>0$ and real numbers $y, z$ in the range $\left(R_{\varepsilon}\right)$, we define $s_{0}=$ $s_{0}(y, z)$ as the unique solution to the equation

$$
\varphi^{\prime}\left(s_{0}\right)=-\log y=-u \mathscr{L}
$$

satisfying

$$
s_{0}=\sigma_{0}+i t_{0}, \quad \varepsilon / 2<\sigma_{0}<1-3 / \mathscr{L}, \quad 0<t_{0}<2 \pi / \mathscr{L},
$$

and we set

$$
\rho(y, z)=e^{\gamma} F\left(z ; s_{0}\right) y^{s_{0}-1} / s_{0} \sqrt{\pi u / 2}
$$

where $u$ and $\mathscr{L}$ are defined by (3.1). (The existence and uniqueness of $s_{0}$ under the stated conditions is shown in Proposition 3.1 below.) Finally, we introduce a "smoothed" form of $r(y, z)$ by setting

$$
r(y, z ; \lambda)=\frac{\sqrt{\lambda}}{\sqrt{2 \pi}} \int_{-\infty}^{\infty} r\left(y e^{v}, z\right) e^{-\lambda v^{2} / 2} d v
$$

where $\lambda$ is a positive real parameter. We then have:

Theorem C. Let $\varepsilon>0$ be fixed. For $(y, z)$ in the range $\left(R_{\varepsilon}\right)$ and $\log u \leq \lambda \leq$ $\exp \left(\mathscr{L}^{3 / 2-\varepsilon}\right)$, we have

$$
r(y, z ; \lambda)=\operatorname{Re} \rho(y, z)+O\left(\frac{|\rho(y, z)|}{\log u}\right),
$$


where $u$ and $\mathscr{L}$ are defined by (3.1) and the implied constant depends at most on $\varepsilon$.

For the proof of this result we use the saddle point method. Its basic idea is to write $r(y, z)$ as a Perron integral over $F(s) y^{s-1} / s$ along a vertical line segment whose abscissa is $\operatorname{Re} s_{0}$. The main contribution to the integral then comes from small neighborhoods of the points $s_{0}$ and $\bar{s}_{0}$ that are saddle points of the function $F(s) y^{s-1}$, and integration over these neighborhoods produces the main term $\operatorname{Re} \rho(y, z)$ in (3.11).

This approach is very similar to the one used by Hildebrand and Tenenbaum $[\mathrm{HT}]$ in the "dual" problem of estimating the function:

$$
\psi(y, z)=\#\{n \leq y: p \mid n \Rightarrow p \leq z\} .
$$

In fact, the main term in (3.7) is formally almost identical to the main term in their asymptotic formula for $\psi(y, z)$, except that different Dirichlet series are involved. There are two important aspects in which the estimation of $r(y, z)$ differs from that of $\psi(y, z)$ however. The first is that, while in the case of $\psi(y, z)$ there is a single dominating saddle point on the real line, the function $F(s) y^{s-1}$ does not have a real saddle point, but rather two saddle points $s_{0}$ and $\bar{s}_{0}$ off the real line whose contributions to the integral are complex conjugates. (The function has other saddle points as well, but their contribution turns out to be negligible.) This explains the appearance of the real part function in (3.11).

The second difference between the estimations of $r(y, z)$ and $\psi(y, z)$ is that in the case of $\psi(y, z)$, the generating Dirichlet series can be bounded trivially on any vertical line by its value at the abscissa. This is not possible in the case of $r(y, z)$ and, as a result, the estimation of the integrand in the Perron integral at large heights becomes a nontrivial problem that prevents us from obtaining a formula of the type (3.11) for $r(y, z)$ if $u$ is large, say a fixed power of $z$. In order to obtain a better convergence in the Perron integral, we have to work with the smoother functions $r(y, z ; \lambda)$ rather than with $r(y, z)$ itself.

To complement Theorem $\mathrm{C}$, we prove the following results, which give estimates for the implicitly defined quantities $s_{0}$ and $\rho(y, z)$ in the main term of (3.11). Here $\varepsilon$ is an arbitrary given positive number, and the implied constants depend at most on $\varepsilon$.

Proposition 3.1. If $(y, z)$ is in the range $\left(R_{\varepsilon}\right)$, then the equation (3.9) has a unique solution $s_{0}=s_{0}(y, z)$ satisfying $(3.10)$, and we have

$$
\begin{gathered}
s_{0}(y, z)=1-\frac{\xi_{u}}{\mathscr{L}}+i \frac{\pi}{\mathscr{L}}\left(1+\frac{1}{\xi_{u}}\right)+O\left(\frac{1}{\mathscr{L} \log ^{2} u}\right), \\
\frac{\partial}{\partial u} s_{0}\left(z^{u}, z\right)=-\frac{1}{\mathscr{L} u}\left(1+O\left(\frac{1}{\log u}\right)\right) .
\end{gathered}
$$


Proposition 3.2. Suppose $(y, z)$ is in the range $\left(R_{\varepsilon}\right)$. Then we have

$$
\begin{gathered}
\rho(y, z)=\exp \left\{-u \xi_{u}+O(u)\right\} \\
\rho\left(z^{u-v}, z\right)=\rho\left(z^{u}, z\right) \exp \left\{v\left(\xi_{u}-i \pi\right)+O\left(\frac{v}{\log u}\right)+O\left(\frac{v^{2}}{u}\right)\right\} \\
(0 \leq v \leq(1-\varepsilon) u), \\
\rho\left(\tilde{z}^{u}, \tilde{z}\right)=\rho\left(z^{u}, z\right) \exp \left\{O\left(\frac{u}{z}\left(|E(\tilde{z}, z)|+\frac{\tilde{z}-z}{\mathscr{L}^{2}}+z^{\varepsilon}\right)\right)\right\}
\end{gathered}
$$

$$
\rho\left(\tilde{z}^{u}, \tilde{z}\right)=\rho\left(z^{u}, z\right) \exp \{O(u / \mathscr{L})\} \quad(\tilde{z} \geq z)
$$

where

$$
E(\tilde{z}, z)=\sum_{z<p \leq \tilde{z}} \log p-(\tilde{z}-z)
$$

Theorem B2 is proven in $\S 4$. Propositions 3.1 and 3.2 and Theorem $\mathrm{C}$ are proven in $\S \S 5-8$. In $\S \S 9$ and 10 we deduce Theorems B1 and B3 from Theorem $\mathrm{C}$ and the two propositions. We conclude this section by deriving two simple corollaries of Theorem $\mathrm{C}$ and then reducing Theorems $\mathrm{B} 1$ and $\mathrm{B} 3$ to the range $u \geq u_{0}$, where $u_{0}$ is arbitrary. First we show that the estimates of Theorem $\mathrm{C}$ hold with $r(y, z)$ in place of $r(y, z ; \lambda)$, provided $u=\log y / \log z$ is not too large.

Corollary 3.1. Let $\varepsilon>0$ and $(y, z)$ be in the range $\left(R_{\varepsilon}\right)$, and suppose that $u \leq \mathscr{L}^{3 / 2-\varepsilon}$. Then we have

$$
r(y, z)=\operatorname{Re} \rho(y, z)+O(|\rho(y, z)| / \log u) .
$$

To deduce this result, we apply Theorem $C$ with $\varepsilon$ replaced by $\varepsilon / 2$ and $\lambda=\exp \left(\mathscr{L}^{(3-\varepsilon) / 2}\right)$. Without loss of generality, we may assume that $\varepsilon<1 / 2$. Using the estimates:

$$
r\left(y e^{v}, z\right)=r(y, z)+O((|v|+1 / y) \mathscr{L}) \quad(|v| \leq 1)
$$

and

$$
r\left(y^{\prime}, z\right)=O(\mathscr{L}) \quad\left(y^{\prime}>0\right),
$$

which easily follow from the definition of $r(y, z)$, we get

$$
\begin{aligned}
r(y, z ; \lambda)= & \frac{r(y, z) \sqrt{\lambda}}{\sqrt{2 \pi}} \int_{-1}^{1} e^{-\lambda v^{2} / 2} d v+O\left(\mathscr{L} \sqrt{\lambda} \int_{-1}^{-1}\left(|v|+\frac{1}{y}\right) e^{-\lambda v^{2} / 2} d v\right) \\
& +O\left(\mathscr{L} \sqrt{\lambda} \int_{1}^{\infty} e^{-\lambda v^{2} / 2} d v\right) .
\end{aligned}
$$

The error terms here can be bounded by

$$
\ll \frac{\mathscr{L}}{\sqrt{\lambda}}+\frac{\mathscr{L}}{y}+\mathscr{L} e^{-\lambda / 2} \ll \exp \left\{-u^{1+\varepsilon / 3}\right\} \ll \frac{\rho(y, z)}{\log u}
$$


if we use the definition of $\lambda$, the bound $u \leq \mathscr{L}^{3 / 2-\varepsilon}$, and the estimates (3.14) and (3.2) for $\rho(y, z)$ and $\xi_{u}$. Moreover, the integral in the main term equals

$$
\int_{-1}^{1} e^{-\lambda v^{2} / 2} d v=\frac{\sqrt{2 \pi}}{\sqrt{\lambda}}\left(1+O\left(e^{-\lambda / 2}\right)\right)=\frac{\sqrt{2} \pi}{\lambda}\left(1+O\left(\frac{1}{\mathscr{L}}\right)\right) .
$$

Thus,

$$
r(y, z ; \lambda)=r(y, z)\left(1+O\left(\frac{1}{\log u}\right)\right)+O\left(\frac{|\rho(y, z)|}{\log u}\right)
$$

and the estimate of the corollary follows from (3.11).

We note that under the Riemann hypothesis, $\lambda$ can be taken as large as $\exp \left(z^{1 / 2-\varepsilon}\right)$ in Theorem $C$, and the estimate of the corollary can be shown to hold in the range $\left(R_{1 / 2+\varepsilon}\right)$ for any $\varepsilon>0$. By appealing to Corollary 3.1 instead of Theorem C in the proof of Theorems B1 and B3, one can then show that, under the Riemann Hypothesis, one can take $y_{ \pm}=y$ in (3.7), as we remarked earlier.

Our second corollary concerns the asymptotic behavior of the Buchstab function $\omega(u)$, defined as the continuous solution of the system

$$
\begin{gathered}
\omega(u)=1 / u \quad(1 \leq u \leq 2), \\
(u w(u))^{\prime}=\omega(u-1) \quad(u>2) .
\end{gathered}
$$

This function was introduced by Buchstab [Bu] who showed that

$$
\lim _{z \rightarrow \infty} r\left(z^{u}, z\right)=e^{\gamma}\left(\omega(u)-e^{-\gamma}\right)
$$

holds for every $u>1$, where $\gamma$ is Euler's constant. Buchstab [Bu] also obtained the estimate:

$$
\omega(u)=e^{-\gamma}+O\left(\exp \left\{-u \xi_{u}+O(u)\right\}\right)
$$

In view of this relation it is natural to consider the function

$$
W(u)=\omega(u)-e^{-\gamma} .
$$

Maier [Ma] showed that $W(u)$ changes sign in every interval of length 1 . Cheer and Goldston [CG] proved that $W(u)$ has at most two sign changes in any interval of length 1 . Furthermore they showed that

$$
\max _{v \geq u} W(v)=\max _{u \leq v \leq u+2} W(v), \quad \min _{v \geq u} W(v)=\min _{u \leq v \leq u+2} W(v) .
$$

Theorem $\mathrm{C}$ and Proposition 3.2 enable us to describe the behavior of $W(u)$ more precisely as follows.

Corollary 3.2. There exists a decreasing function $W_{0}(u)$ satisfying

$$
W_{0}(u)=\exp \left\{-u \xi_{u}+O(u)\right\} \quad\left(u \geq u_{0}\right)
$$

and a function $\theta(u)$ satisfying

$$
\theta(u+t)-\theta(u)=t \pi(1+O(1 / \log u)) \quad\left(u \geq u_{0}, 0 \leq t \leq 1\right)
$$


such that, as $u \rightarrow \infty$,

$$
W(u)=W_{0}(u)\{\cos \theta(u)+O(1 / \log u)\} .
$$

This result clearly exhibits the oscillatory behavior of $W(u)$. Aside from a "decay factor" $W_{0}(u)$, the function $W(u)$ behaves like a trigonometric function with period 2. An immediate consequence of this behavior and the estimate (3.19) is that

$$
\max _{u \leq v \leq u+2} W(v)=\exp \left\{-u \xi_{u}+O(u)\right\}
$$

and similarly,

$$
\min _{u \leq v \leq u+2} W(v)=-\exp \left\{-u \xi_{u}+O(u)\right\}
$$

To prove the corollary, we note that, by Buchstab's result (3.18) and Theorem C,

$$
\begin{aligned}
W(u) & =e^{-\gamma} \lim _{z \rightarrow \infty} r\left(z^{u}, z\right) \\
& =e^{-\gamma} \lim _{z \rightarrow \infty}\left\{\operatorname{Re} \rho\left(z^{u}, z\right)+O\left(\left|\rho\left(z^{u}, z\right)\right| / \log u\right)\right\},
\end{aligned}
$$

provided $u$ is sufficiently large. Also, the estimates (3.17), (3.14), and (3.15) of Proposition 3.2 imply that the limit

$$
l(u)=\lim _{z \rightarrow \infty} \rho\left(z^{u}, z\right)
$$

exists for every sufficiently large $u$ and satisfies

$$
|l(u)|=\exp \left\{-u \xi_{u}+O(u)\right\}
$$

and

$$
\arg l(u+t)-\arg l(u)=t \pi(1+O(1 / \log u)), \quad\left(u \geq u_{0}, 0 \leq t \leq 1\right),
$$

where $\arg l(u)$ denotes a continuous branch of the argument of $l(u)$. Taking $W_{0}(u)=e^{-\gamma}|l(u)|$ and $\theta(u)=\arg l(u)$ then yields the asserted estimates.

As already mentioned, the results of Theorems B1 and B3 follow fairly easily in the range $1+\varepsilon \leq u \leq u_{0}$ for fixed $\varepsilon$ and $u_{0}$. In this range the formula (3.18) of Buchstab still holds with $r$ replaced by $r_{k}$ (subject to (3.5)). In the case $u \geq 2$, this is an immediate consequence of [FG, Lemma 5]; in the case $1+\varepsilon \leq u \leq 2$, that proof needs only a slight modification. An easy variation of Buchstab's original arguments shows that (3.18) also holds with $r$ replaced by $\bar{r}^{(p)}$. Theorems B1 and B3 then follow for $1+\varepsilon \leq u \leq u_{0}$ from these facts in conjunction with Maier's result that $W(u)$ changes sign in every interval of length 1 [Ma, Lemma 4].

Note. We take this opportunity to correct an error in [FG]. Professor Warlimont has kindly pointed out to us an omission from the identity in the proof of Lemma 5 in [FG]: specifically that the contribution $\sum_{x / y<n \leq x}^{\prime} 1$ was overlooked. As before, $\Sigma^{\prime}$ denotes a sum over integers all of whose prime factors divide $q$, and $q$ is assumed to have fewer than $y^{f}$ distinct prime factors where $f=f(y)$ tends to zero as $y \rightarrow \infty$ with $f(y)>3 \log _{2} y / \log y$. 
In order to complete the proof of Lemma 5 it suffices to establish, for $x \geq$ $y \geq y_{0}$, the estimate

$$
\sum_{n \leq x}^{\prime} 1 \ll x / \log ^{3} y+\frac{q}{\varphi(q)} \frac{x f}{\log x} .
$$

By first considering the contribution to the sum of those $n \leq x^{1 / 2}$, then those $n$ divisible by some prime $>y^{2 f}$, and finally the remaining $n$, we find that (compare Exercise 01 of [HaT]) for $\sigma=1 / f \log y$,

$$
\begin{aligned}
\sum_{n \leq x}^{\prime} 1 & \ll x^{1 / 2}+\nu(q) x / y^{2 f}+\sum_{x^{1 / 2}<n \leq x}^{*}\left(n / x^{1 / 2}\right)^{\sigma} \\
& \ll x / \log ^{3} y+x^{-\sigma / 2} \sum_{n \leq x}^{*} n^{\sigma},
\end{aligned}
$$

where $\sum^{*}$ denotes the restriction to those $n$ divisible only by prime divisors of $q$ that are $\leq y^{2 f}$. By a Theorem of Halberstam and Richert [HaT, Theorem $00]$, we have

$$
\begin{aligned}
\sum_{n \leq x}^{*} n^{\sigma} & \ll \frac{x}{\log x} \sum_{n \geq 1}^{*} \frac{1}{n^{1-\sigma}} \ll \frac{q}{\varphi(q)} \frac{x}{\log x} \exp \left\{\sum_{p \leq y^{2 f}}\left(\frac{1}{p^{1-\sigma}}-\frac{1}{p}\right)\right\} \\
& \ll \frac{q}{\varphi(q)} \frac{x}{\log x} .
\end{aligned}
$$

Therefore (3.21) and (3.22) yield (3.20), since $x^{-\sigma / 2} \ll f \log y / \log x$, and Lemma 5 follows as stated.

\section{Proof of Theorem B2}

For $m$ a positive integer, let

$$
s(m)=\sum_{p \mid m} \frac{1}{p}, \quad \nu(m)=\sum_{p \mid m} 1 .
$$

We require the following lemma:

Lemma 4.1. For any positive integers $m, j$ with $m$ squarefree and $s(m) \leq j / 2$, we have

$$
\sum_{\substack{d \mid m \\ \nu(d) \geq j+1}} \frac{1}{d} \leq \frac{2 s(m)}{j} \sum_{\substack{d \mid m \\ \nu(d)=j}} \frac{1}{d} .
$$

Proof. We first show that, for every integer $i \geq 0$,

$$
\sum_{\substack{d \mid m \\ \nu(d)=j+i}} \frac{1}{d} \leq \frac{j !}{(j+i) !} s^{i}(m) \sum_{\substack{d \mid m \\ \nu(d)=j}} \frac{1}{d} .
$$

We observe that any divisor $d$ of $m$ with $\nu(d)=j+i$ can be written in 
the form $d=\delta p_{1} \cdots p_{i}$ (where $\nu(\delta)=j$ and the $p_{\mu}$ are prime) in $\left(\begin{array}{c}j+i \\ j\end{array}\right) i !=$ $(j+i) ! / j$ ! distinct ways, if we distinguish amongst different orderings of the $p_{\mu}$.

By multiplying out and collecting terms on the right side, we get (4.2). To prove (4.1) we note that the left side is obtained by summing over $i$ the left side of (4.2). The application of (4.2) thus yields

$$
\begin{aligned}
\sum_{\substack{d \mid m \\
\nu(d) \geq j+1}} \frac{1}{d} & \leq \sum_{\substack{d \mid m \\
\nu(d)=j}} \frac{1}{d} \sum_{i \geq 1} \frac{j !}{(j+i) !} s^{i}(m) \\
& \leq \sum_{\substack{d \mid m \\
\nu(d)=j}} \frac{1}{d} \sum_{i \geq 1}\left(\frac{s(m)}{j}\right)^{i}
\end{aligned}
$$

and since $s(m) \leq j / 2$, this gives (4.1).

Proof of Theorem B2. If $d \mid P$ with $\nu(d) \geq j$, then $d>(z-h)^{j} \geq y$, so $[y / d]=0$. Now, since

$$
r_{P}(y)=\sum_{\substack{n \leq y \\(n, \bar{P})=1}} 1-\frac{\varphi(P)}{P} y=\sum_{d \mid P} \mu(d)\left(\left[\frac{y}{d}\right]-\frac{y}{d}\right),
$$

we have

$$
\left|r_{P}(y)+(-1)^{j} y \sum_{\substack{d \mid P \\ \nu(d)=j}} \frac{1}{d}\right| \leq y \sum_{\substack{d \mid P \\ \nu(d) \geq j+1}} \frac{1}{d}+\sum_{\substack{d \mid P \\ \nu(d) \leq j-1}} 1 .
$$

Since $s(P) \leq \pi(z) /(z-h) \leq 1 / 8$ for $z>z_{0}$, we may use Lemma 4.1 to estimate the first term on the right-hand side of (4.3). Since $\left(\begin{array}{c}k \\ i-1\end{array}\right) \leq \frac{1}{2}\left(\begin{array}{c}k \\ i\end{array}\right)$ for $i \leq k / 3$, the second term is

$$
\begin{aligned}
\sum_{\substack{d \mid P \\
\nu(d) \leq j-1}} 1 & =\sum_{i=0}^{j-1}\left(\begin{array}{l}
k \\
i
\end{array}\right) \leq 2\left(\begin{array}{c}
k \\
j-1
\end{array}\right) \\
& \leq \frac{y}{2 z^{j}}\left(\begin{array}{l}
k \\
j
\end{array}\right) \leq \frac{y}{2} \sum_{\substack{d \mid P \\
\nu(d)=j}} \frac{1}{d} .
\end{aligned}
$$

Substituting these in (4.3) we complete the proof of Theorem B2.

\section{Proof of Proposition 3.1}

We begin with a lemma, which is needed again in later sections.

Lemma 5.1. Let $\varepsilon>0$ and $k \geq 1$. Uniformly for $z \geq 2$ and $s=\sigma+$ it in the range

$$
\varepsilon \leq \sigma<1-1 / \mathscr{L}, \quad|t| \leq 1
$$


we have

$$
\begin{gathered}
\varphi(z ; s)=-\int_{1}^{(1-s) \mathscr{L}} \frac{e^{w}}{w} d w+O\left(\frac{z^{1-\sigma}}{\mathscr{L}^{k}}\right)+O(1), \\
\varphi^{\prime}(z ; s)=\left(1+O\left(\frac{1}{\mathscr{L}^{k}}\right)\right) \frac{z^{1-s}}{1-s}+O(1), \\
\varphi^{\prime \prime}(z ; s)=\left(1+O\left(\frac{1}{\mathscr{L}^{k}}\right)\right) \frac{z^{1-s}(1-(1-s) \mathscr{L})}{(1-s)^{2}}+O(1), \\
\left|\varphi^{\prime \prime \prime}(z ; s)\right| \leq\left(1+O\left(\frac{1}{(1-\sigma) \mathscr{L}}\right)\right) \frac{z^{1-\sigma}}{1-\sigma} \mathscr{L}^{2},
\end{gathered}
$$

where $\mathscr{L}=\log z$ and the implied constants depend at most on $\varepsilon$ and $k$.

Proof. Differentiating $\varphi(z ; s)=\log \left(\prod_{p<z}\left(1-1 / p^{s}\right) \zeta(s)\right)$, we obtain

$$
\varphi^{\prime}(s)=\sum_{p<z} \frac{\log p}{p^{s}-1}+\frac{\zeta^{\prime}(s)}{\zeta(s)} .
$$

In the range (5.1) we have

$$
\frac{\zeta^{\prime}(s)}{\zeta(s)}=\frac{1}{1-s}+O(1)
$$

and

$$
\begin{aligned}
\sum_{p<z} \frac{\log p}{p^{s}-1} & =\sum_{p<z} \frac{\log p}{p^{s}}+O\left(\sum_{p<z} \frac{\log p}{p^{2 \sigma}}\right) \\
& =\left(1+O\left(\frac{1}{\mathscr{L}^{k}}\right)\right) \int_{1}^{z} \frac{d u}{u^{s}}+O(1)+O\left(z^{1-2 \sigma} \log z\right) \\
& =\left(1+O\left(\frac{1}{\mathscr{L}^{k}}\right)\right) \frac{z^{1-s}-1}{1-s}+O(1),
\end{aligned}
$$

using partial summation and the prime number theorem with logarithmic error term. Substituting these estimates into (5.6) yields (5.3). The estimates (5.4) and (5.5) can be proved in the same way.

To obtain (5.2), we note that by (5.3),

$$
\begin{aligned}
\varphi(z ; s)-\varphi\left(z ; 1-\frac{1}{\mathscr{L}}\right)= & \int_{1-1 / \mathscr{L}}^{s} \varphi^{\prime}\left(z ; s^{\prime}\right) d s^{\prime} \\
= & -\frac{1}{\mathscr{L}} \int_{1}^{(1-s) \mathscr{L}} \varphi^{\prime}\left(z ; 1-\frac{w}{\mathscr{L}}\right) d w \\
= & -\int_{1}^{(1-s) \mathscr{L}} \frac{e^{w}}{w} d w \\
& +O\left(\frac{1}{\mathscr{L}^{k+1}} \int_{1}^{(1-s) \mathscr{L}}\left|\frac{e^{w}}{w}\right||d w|\right)+O(1) \\
= & -\int_{1}^{(1-s) \mathscr{L}} \frac{e^{w}}{w} d w+O\left(\frac{z^{1-\sigma}}{\mathscr{L}^{k}}\right)+O(1) .
\end{aligned}
$$


Since

$$
\begin{aligned}
\varphi\left(z ; 1-\frac{1}{\mathscr{L}}\right) & =\sum_{p<z} \log \left(1-\frac{1}{p^{1-1 / \mathscr{L}}}\right)+\log \zeta\left(1-\frac{1}{\mathscr{L}}\right) \\
& =-\sum_{p<z} \frac{1}{p^{1-1 / \mathscr{L}}}+\log \mathscr{L}+O(1) \\
& =-\sum_{p<z} \frac{1}{p}+\log _{2} z+O(1)=O(1),
\end{aligned}
$$

this implies the desired estimate (5.2).

We now proceed to prove Proposition 3.1. We assume that $(y, z)$ lies in the range $\left(R_{\varepsilon}\right)$ with a given parameter $\varepsilon>0$ and sufficiently large constants $z_{0}$ and $u_{0}$. We first establish the existence and uniqueness of $s_{0}(y, z)$ as a solution to (3.9) in the range (3.10). To this end it suffices to show that (i) for every $\sigma \in[\varepsilon / 2,1-3 / \mathscr{L}]$, there exists a unique number $t=t(\sigma) \in(0,2 \pi / \mathscr{L})$ such that $\varphi^{\prime}(\sigma+i t)$ is real and negative, and (ii) the function $f(\sigma)=\varphi^{\prime}(\sigma+i t(\sigma))$ is strictly increasing in the interval $[\varepsilon / 2,1-3 / \mathscr{L}]$ with $f(\varepsilon / 2)<-z^{1-\varepsilon} \mathscr{L}$ and $f(1-3 / \mathscr{L})=O(\mathscr{L})$.

For the proof of (i) we observe first that, by $(5.6), \varphi(\sigma)$ is positive for $0<\sigma<1$, since $\zeta^{\prime}(\sigma) / \zeta(\sigma)>0$ in this interval. The assertion (i) follows if we can show that

$$
\frac{d}{d t} \arg \varphi^{\prime}(\sigma+i t) \in\left(\frac{-3}{2} \mathscr{L}, \frac{-1}{2} \mathscr{L}\right)
$$

holds for $\varepsilon / 2 \leq \sigma \leq 1-3 / \mathscr{L}$ and $0 \leq t \leq 2 \pi / \mathscr{L}$. Using Lemma 5.1 with $\varepsilon / 2$ in place of $\varepsilon$ and $k=1$, we see that in this range

$$
\begin{aligned}
\frac{d}{d t} \arg \varphi^{\prime}(\sigma+i t) & =\frac{d}{d t} \operatorname{Im} \log \varphi^{\prime}(\sigma+i t)=\operatorname{Re} \frac{\varphi^{\prime \prime}(\sigma+i t)}{\varphi^{\prime}(\sigma+i t)} \\
& =\operatorname{Re}\left(1+O\left(\frac{1}{\mathscr{L}}\right)\right)\left(\frac{1}{1-s}-\mathscr{L}\right) \\
& =\left(1+O\left(\frac{1}{\mathscr{L}}\right)\right)\left(\frac{1-\sigma}{(1-\sigma)^{2}+t^{2}}-\mathscr{L}\right)
\end{aligned}
$$

Since

$$
0<\frac{1-\sigma}{(1-\sigma)^{2}+t^{2}} \leq \frac{1}{1-\sigma} \leq \frac{\mathscr{L}}{3}
$$

for $0<\sigma<1-3 / \mathscr{L},(5.7)$ follows if $z$ (and hence $\mathscr{L}$ ) is sufficiently large, as we may assume. Since $\arg \varphi^{\prime}(\sigma+i t)$ is a continuously differentiable function of $(\sigma, t)$ in the range under consideration, the function $t(\sigma)$ defined by (i) is continuously differentiable as well.

To prove (ii), we note that the function

$$
f^{\prime}(\sigma)=\varphi^{\prime \prime}(\sigma+i t(\sigma))\left(1+i t^{\prime}(\sigma)\right)
$$

is real-valued, continuous, and has no zeros in the interval $[\varepsilon / 2,1-3 / \mathscr{L}]$, since by $(5.4), \varphi^{\prime \prime}(\sigma+i t(\sigma)) \neq 0$ in this interval. Thus $f(\sigma)$ is either monotonically 
decreasing or monotonically increasing, as $\sigma$ increases from $\varepsilon / 2$ to $1-3 / \mathscr{L}$. By Lemma 5.1 and the definition of $t(\sigma)$, we have

$$
\begin{aligned}
f(\varepsilon / 2) & =\varphi^{\prime}(\varepsilon / 2+i t(\varepsilon / 2)) \\
& =-\left|\varphi^{\prime}(\varepsilon / 2+i t(\varepsilon / 2))\right| \\
& =\frac{-z^{1-\varepsilon / 2}}{|1-\varepsilon / 2-i t(\varepsilon / 2)|}\left(1+O\left(\frac{1}{\mathscr{L}}\right)\right),
\end{aligned}
$$

which is $<-z^{1-\varepsilon} \mathscr{L}$, if $z$ is sufficiently large. Similarly,

$$
f\left(1-\frac{3}{\mathscr{L}}\right)=\frac{-z^{3 / \mathscr{L}}}{|(3 / \mathscr{L})-i t(1-3 / \mathscr{L})|}\left(1+O\left(\frac{1}{\mathscr{L}}\right)\right),
$$

which is of order $O(\mathscr{L})$. Assertion (ii) now follows.

It remains to prove estimates (3.12) and (3.13). Setting $s_{0}=1-s_{1} / \mathscr{L}$, $s_{1}=\sigma_{1}+i t_{1}$, we can restate $(3.12)$ as

$$
\sigma_{1}=\xi_{u}+O\left(\frac{1}{\log ^{2} u}\right), \quad t_{1}=-\pi\left(1+\frac{1}{\xi_{u}}\right)+O\left(\frac{1}{\log ^{2} u}\right) .
$$

From (3.9) and (5.3) we get

$$
-u \mathscr{L}=\varphi^{\prime}\left(z ; s_{0}\right)=\left(1+O\left(\frac{1}{\mathscr{L}^{2}}\right)\right) \frac{e^{s_{1}}}{s_{1}} \mathscr{L}+O(1),
$$

so that

$$
\frac{e^{s_{1}}}{s_{1}}=-u\left(1+O\left(\frac{1}{\mathscr{L}^{2}}\right)+O\left(\frac{1}{u \mathscr{L}}\right)\right) .
$$

Since $\left|e^{S_{1}}\right|=e^{\sigma_{1}}$ and

$$
\left|s_{1}\right|=\left|\sigma_{1}+i t_{1}\right|=\sigma_{1}\left(1+O\left(t_{1}^{2} / \sigma_{1}^{2}\right)\right)=\sigma_{1}\left(1+O\left(1 / \sigma_{1}^{2}\right)\right),
$$

we deduce from (5.9) that

$$
e^{\sigma_{1}} / \sigma_{1} \asymp u,
$$

which implies $\sigma_{1} \gg \log u$ and, by another application of (5.9),

$$
\begin{aligned}
\frac{e^{\sigma_{1}}}{\sigma_{1}} & =u\left(1+O\left(\frac{1}{\mathscr{L}^{2}}\right)+O\left(\frac{1}{u \mathscr{L}}\right)+O\left(\frac{1}{\sigma_{1}^{2}}\right)\right) \\
& =u\left(1+O\left(\frac{1}{\log ^{2} u}\right)\right) .
\end{aligned}
$$

Now note that the function $e^{x} / x$ is monotonically increasing in $x \geq 1$ and that, by the definition of $\xi_{u}$,

$$
\begin{aligned}
\frac{e^{\xi_{u}+\delta}}{\xi_{u}+\delta} & =\frac{\left(u \xi_{u}+1\right)\left(1+\delta+O\left(\delta^{2}\right)\right)}{\xi_{u}\left(1+\delta / \xi_{u}\right)} \\
& =u\left(1+\left(1-1 / \xi_{u}\right) \delta+O\left(\delta^{2}\right)+O\left(1 / u \xi_{u}\right)\right) \quad(|\delta| \leq 1) .
\end{aligned}
$$


Comparison of (5.10) and (5.11) shows that $\sigma_{1}=\xi_{u}+\delta$ with $\delta=O\left(1 / \log ^{2} u\right)$, as asserted in (5.8).

To prove the second estimate of (5.8), we take the argument on both sides of (5.9), keeping in mind that by $(3.10), t_{1}=-t_{0} \mathscr{L}$ lies in the interval $(-2 \pi, 0)$. This yields the equation

$$
-\pi+O\left(\frac{1}{\mathscr{L}^{2}}\right)+O\left(\frac{1}{u \mathscr{L}}\right)=t_{1}-\arctan \frac{t_{1}}{\sigma_{1}}=t_{1}-\frac{t_{1}}{\sigma_{1}}+O\left(\frac{1}{\sigma_{1}^{2}}\right),
$$

which implies the desired estimate

$$
t_{1}=-\pi\left(1+\frac{1}{\sigma_{1}}\right)+O\left(\frac{1}{\log ^{2} u}\right)=-\pi\left(1+\frac{1}{\xi_{u}}\right)+O\left(\frac{1}{\log ^{2} u}\right) .
$$

Finally, to obtain the estimate (3.13) for the derivative of $s_{0}$, we differentiate both sides of the equation $\varphi^{\prime}\left(z ; s_{0}\right)=-u \mathscr{L}$ with respect to $u$. In this way, we get

$$
\varphi^{\prime \prime}\left(z ; s_{0}\right) \frac{\partial}{\partial u} s_{0}\left(z^{u}, z\right)=-\mathscr{L},
$$

so that

$$
\begin{aligned}
\frac{\partial}{\partial u} s_{0}\left(z^{u}, z\right) & =-\frac{\mathscr{L}}{\varphi^{\prime \prime}\left(z ; s_{0}\right)} \\
& =\frac{\left(1-s_{0}\right)^{2} \mathscr{L}}{z^{1-s_{0}}\left(\left(1-s_{0}\right) \mathscr{L}-1\right)}\left(1+O\left(\frac{1}{\mathscr{L}}\right)\right) \\
& =\frac{1}{\varphi^{\prime}\left(z ; s_{0}\right)}\left(1+O\left(\frac{1}{\left(1-\sigma_{0}\right) \mathscr{L}}\right)\right)=-\frac{1}{u \mathscr{L}}\left(1+O\left(\frac{1}{\log u}\right)\right)
\end{aligned}
$$

by the estimates of Lemma 5.1 and (3.12). This proves (3.13).

\section{Proof of Proposition 3.2}

First we consider the behavior of $\rho(y, z)$ when $z$ is fixed and $y=z^{u}$ varies. Setting

$$
\begin{aligned}
f(u) & =\log \rho\left(z^{u}, z\right)=\log \frac{e^{\gamma} y^{s_{0}-1} F\left(s_{0}\right)}{s_{0} \sqrt{\pi u / 2}} \\
& =\left(s_{0}-1\right) u \mathscr{L}+\varphi\left(s_{0}\right)-\log s_{0}-\frac{1}{2} \log u+\log \frac{e^{\gamma}}{\sqrt{\pi / 2}},
\end{aligned}
$$

we can rewrite the first two estimates of Proposition 3.2 as

$$
f(u)=-u \xi_{u}+O(u)
$$

and

(6.3) $f(u-v)=f(u)+v\left(\xi_{u}-i \pi\right)+O\left(\frac{v}{\log u}\right)+O\left(\frac{v^{2}}{u}\right)$

$$
(0 \leq v \leq u(1-\varepsilon))
$$


respectively, and it suffices to show that these latter two estimates hold for $u_{0} \leq u \leq z^{1-\varepsilon}$.

Taking the derivative with respect to $u$ in (6.1) we obtain

$$
f^{\prime}(u)=s_{0}^{\prime} u \mathscr{L}+\left(s_{0}-1\right) \mathscr{L}+\varphi^{\prime}\left(s_{0}\right) s_{0}^{\prime}-s_{0}^{\prime} / s_{0}-1 / 2 u,
$$

where for brevity we have set $s_{0}^{\prime}=(\partial / \partial u) s_{0}\left(z^{u}, z\right)$. Since $\varphi^{\prime}\left(s_{0}\right)=-u \mathscr{L}$ by (3.9) and

$$
\begin{aligned}
\left(s_{0}-1\right) \mathscr{L} & =-\xi_{u}+i \pi+O(1 / \log u), \\
\frac{s_{0}^{\prime}}{s_{0}} & =\frac{-1}{u \mathscr{L} s_{0}}\left(1+O\left(\frac{1}{\log u}\right)\right)=O\left(\frac{1}{\log u}\right)
\end{aligned}
$$

by Proposition 3.1, it follows that in the range $\left(R_{\varepsilon}\right)$

$$
f^{\prime}(u)=-\xi_{u}+i \pi+O(1 / \log u) .
$$

This implies, for $0 \leq v \leq(1-\varepsilon) u$,

$$
\begin{aligned}
f(u-v) & =f(u)-\int_{u-v}^{u} f^{\prime}(w) d w \\
& =f(u)+\int_{u-v}^{u}\left(\xi_{w}-i \pi+O\left(\frac{1}{\log w}\right)\right) d w \\
& =f(u)+v\left(\xi_{u}-i \pi\right)+O\left(v^{2} / u\right)+O(v / \log u),
\end{aligned}
$$

and hence (6.3), after adjusting the constant $u_{0}$ in $\left(R_{\varepsilon}\right)$, if necessary. Here we have used the estimate

$$
\xi_{w}=\xi_{u}+O(|u-w| / u) \quad(|u-w| \leq(1-\delta) u),
$$

which follows from the bound (3.3) for $(d / d u) \xi_{u}$.

To prove (6.2), we apply (6.4) again to obtain

$$
\begin{aligned}
f(u) & =f\left(u_{0}\right)+\int_{u_{0}}^{u} f^{\prime}(w) d w \\
& =f\left(u_{0}\right)-\int_{u_{0}}^{u}\left(\xi_{w}+O(1)\right) d w \\
& =f\left(u_{0}\right)-u \xi_{u}+O(u)
\end{aligned}
$$

then (6.2) follows, if we can show that $f\left(u_{0}\right)=O(1)$. Since, by Proposition 3.1 ,

$$
\left(s_{0}-1\right) \mathscr{L}=-\xi_{u}+O(1),
$$

we see from (6.1) that $f\left(u_{0}\right)=\varphi\left(s_{0}\right)+O(1)$, where $s_{0}=s_{0}\left(z^{u_{0}}, z\right)$. Now, by Lemma 5.1 and Proposition 3.1, we have

$$
\begin{aligned}
\varphi\left(s_{0}\right) & =-\int_{1}^{\left(1-s_{0}\right) \mathscr{L}} \frac{e^{w}}{w} d w+O\left(\frac{z^{1-\sigma_{0}}}{\mathscr{L}^{2}}\right)+O(1) \\
& =O\left(e^{\left(1-\sigma_{0}\right) \mathscr{L}}\right)=O\left(e^{\xi_{u_{0}}}\right)=O(1) .
\end{aligned}
$$


Hence we obtain $f\left(u_{0}\right)=O(1)$ as desired.

It remains to prove estimates (3.16) and (3.17). To this end we consider $\rho\left(z^{u}, z\right)$ as a function of $z$ with $u$ fixed, and define, in analogy to (6.1),

$$
g(z)=\log \rho\left(z^{u}, z\right)=\left(s_{0}-1\right) u \log z+\varphi\left(z ; s_{0}\right)-\log s_{0}+\log \frac{e^{\gamma}}{\sqrt{\pi u / 2}} .
$$

Setting $s_{0}=s_{0}\left(z^{u}, z\right)$ and $\tilde{s}_{0}=s_{0}\left(\tilde{z}^{u}, \tilde{z}\right)$, the asserted estimates can be restated as

$$
|g(\tilde{z})-g(z)| \ll \frac{u}{z}|E(\tilde{z}, z)|+\frac{\Delta u}{\mathscr{L}^{2}}+\frac{u}{z^{1-\varepsilon}} \quad(z \leq \tilde{z} \leq 2 z),
$$

and

$$
|g(\tilde{z})-g(z)| \ll u / \mathscr{L} \quad(\tilde{z} \geq z),
$$

where $\Delta=(\tilde{z}-z) / z$. We shall prove that (6.6) holds in the case

$$
\Delta \leq 1 / \mathscr{L}^{3}
$$

The relation (6.6) for $\mathscr{L}^{-3} \leq \Delta \leq 1$ and the relation (6.7) can thus be deduced by splitting the interval $(z, \tilde{z}]$ into subintervals of the form $\left(z_{1}\right.$, $z_{1}\left(1+\mathscr{L}^{-5}\right)$ ] (plus possibly one interval of shorter length) and by applying (6.6) (with $\varepsilon$ replaced by $\varepsilon / 2$ ) along with the prime number theorem in the form $E\left(z_{1}\left(1+\mathscr{L}^{-5}\right), z_{1}\right) \ll z_{1} \mathscr{L}^{-7}$ to these intervals.

From (6.5) we obtain

$$
g(z)-g(\tilde{z})=R_{1}+R_{2}+R_{3},
$$

where

$$
\begin{aligned}
& R_{1}=u\left(s_{0}-\tilde{s}_{0}\right) \log z+\varphi\left(z ; s_{0}\right)-\varphi\left(z ; \tilde{s}_{0}\right), \\
& R_{2}=\varphi\left(z ; \tilde{s}_{0}\right)-\varphi\left(\tilde{z} ; \tilde{s}_{0}\right)+u\left(1-\tilde{s}_{0}\right) \log (\tilde{z} / z), \\
& R_{3}=\log \left(\tilde{s}_{0} / s_{0}\right) .
\end{aligned}
$$

To prove (6.6), we show that each of the terms $R_{i}$ is bounded by the right-hand side of (6.6).

We first derive an estimate for $\tilde{s}_{0}-s_{0}$. By the definition of $s_{0}$ and $\tilde{s}_{0}$, we have

$$
-u \log \tilde{z}+u \log z=\varphi^{\prime}\left(\tilde{z} ; \tilde{s}_{0}\right)-\varphi^{\prime}\left(z ; s_{0}\right)=R_{4}+R_{5}
$$

with

$$
\begin{aligned}
& R_{4}=\varphi^{\prime}\left(\tilde{z} ; \tilde{s}_{0}\right)-\varphi^{\prime}\left(z ; \tilde{s}_{0}\right)=\sum_{z \leq p<\tilde{z}} \frac{\log p}{p^{\tilde{s}_{0}}-1} \\
& R_{5}=\varphi^{\prime}\left(z ; \tilde{s}_{0}\right)-\varphi^{\prime}\left(z ; s_{0}\right)=\int_{s_{0}}^{\tilde{s}_{0}} \varphi^{\prime \prime}(z ; s) d s .
\end{aligned}
$$


Since

$$
\begin{aligned}
\left|R_{4}\right| & \ll \tilde{z}^{-\tilde{\sigma}_{0}} \sum_{z \leq p<\tilde{z}} \log p \\
& \ll \tilde{z}^{1-\tilde{\sigma}_{0}}\left(\Delta+z^{\varepsilon-1}\right) \\
& \ll e^{\xi_{u}}\left(\Delta+z^{\varepsilon-1}\right) \ll u(\log u)\left(\Delta+z^{\varepsilon-1}\right)
\end{aligned}
$$

by a standard upper bound for primes in short intervals and Proposition 3.1, it follows that

$$
\begin{aligned}
\left|R_{5}\right| & \ll\left|R_{4}\right|+u \log (\tilde{z} / z) \\
& \ll u(\log u)\left(\Delta+z^{\varepsilon-1}\right) .
\end{aligned}
$$

On the other hand, since by Proposition 3.1 and our assumption (6.8),

$$
\begin{aligned}
\tilde{s}_{0}-s_{0} & =\frac{\xi_{u}-i \pi}{\log z}-\frac{\xi_{u}-i \pi}{\log \tilde{z}}+O\left(\frac{1}{\log z \log u}\right) \\
& =O\left(\frac{\log (\tilde{z} / z)}{\mathscr{L}^{2}} \log u\right)+O\left(\frac{1}{\mathscr{L} \log u}\right) \\
& =O\left(\frac{\Delta \log u}{\mathscr{L}^{2}}\right)+O\left(\frac{1}{\mathscr{L} \log u}\right)=O\left(\frac{1}{\mathscr{L} \log u}\right),
\end{aligned}
$$

we obtain from Lemma 5.1 for $s \in\left[s_{0}, \tilde{s}_{0}\right]$

$$
\begin{aligned}
\varphi^{\prime \prime}(z ; s) & =-\left(1+O\left(\frac{1}{\mathscr{L}}\right)\right) \frac{z^{1-s}((1-s) \mathscr{L}-1)}{(1-s)^{2}}+O(1) \\
& =-\left(1+O\left(\frac{1}{\log u}\right)\right) \frac{z^{1-s_{0}} \mathscr{L}}{1-s_{0}} \\
& =\left(1+O\left(\frac{1}{\log u}\right)\right) u \mathscr{L}^{2}
\end{aligned}
$$

so that

$$
\left|R_{5}\right|=\left|\int_{s_{0}}^{\tilde{s}_{0}} \varphi^{\prime \prime}(z ; s) d s\right| \gg u \mathscr{L}^{2}\left|\tilde{s}_{0}-s_{0}\right| .
$$

Comparing this bound with (6.9), we deduce that

$$
\left|\tilde{s}_{0}-s_{0}\right| \ll \frac{\log u}{\mathscr{L}^{2}}\left(\Delta+\frac{1}{z^{1-\varepsilon}}\right) .
$$

Since $R_{3}=\log \left(\tilde{s}_{0} / s_{0}\right)=O\left(\left|\tilde{s}_{0}-s_{0}\right|\right), R_{3}$ is bounded by the right-hand side of (6.6).

To estimate $R_{1}$, we write

$$
\begin{aligned}
\varphi\left(z ; s_{0}\right)-\varphi\left(z ; \tilde{s}_{0}\right) & =-\int_{s_{0}}^{\tilde{s}_{0}} \varphi^{\prime}(z ; s) d s \\
& =-\varphi^{\prime}\left(z ; s_{0}\right)\left(\tilde{s}_{0}-s_{0}\right)-\int_{s_{0}}^{\tilde{s}_{0}} \varphi^{\prime \prime}(z ; s)\left(\tilde{s}_{0}-s\right) d s
\end{aligned}
$$


and we note that by (3.9), the first term on the right-hand side is equal to $u \mathscr{L}\left(\tilde{s}_{0}-s_{0}\right)$. It follows that

$$
\begin{aligned}
\left|R_{1}\right| & =\left|\int_{s_{0}}^{\tilde{s}_{0}} \varphi^{\prime \prime}(z ; s)\left(\tilde{s}_{0}-s\right) d s\right| \\
& \leq\left|\tilde{s}_{0}-s_{0}\right|^{2} \max _{s \in\left[s_{0}, \tilde{s}_{0}\right]}\left|\varphi^{\prime \prime}(z ; s)\right| \\
& \ll \frac{\log ^{2} u}{\mathscr{L}^{4}}\left(\Delta+\frac{1}{z^{1-\varepsilon}}\right)^{2} u \mathscr{L}^{2} \ll \frac{u \log ^{2} u}{\mathscr{L}^{5}}\left(\Delta+\frac{1}{z^{1-\varepsilon}}\right) \ll \frac{\Delta u}{\mathscr{L}^{3}}+\frac{u}{z^{1-\varepsilon}}
\end{aligned}
$$

by (6.11), (6.10), and (6.8). This proves the desired bound for $R_{1}$.

It remains to estimate $R_{2}$. We have

$$
\begin{aligned}
R_{2}= & u\left(1-\tilde{s}_{0}\right) \log \frac{\tilde{z}}{z}-\sum_{z \leq p<\tilde{z}} \log \left(1-\frac{1}{p^{\tilde{s}_{0}}}\right) \\
= & u\left(1-\tilde{s}_{0}\right) \log \frac{\tilde{z}}{z}+\frac{1}{\tilde{z}^{\tilde{s}_{0}} \log \tilde{z}} \sum_{z \leq p<\tilde{z}} \log p \\
& +O\left(\sum_{z \leq p<\tilde{z}}\left|\frac{\log p}{\tilde{z}^{\tilde{s}_{0}} \log \tilde{z}}-\frac{1}{p^{\tilde{s}_{0}}}\right|\right)+O\left(|\tilde{z}-z+1| \tilde{z}^{-2 \tilde{\sigma}_{0}}\right) .
\end{aligned}
$$

The second error term here is of order

$$
\begin{aligned}
& \ll \tilde{z}^{2\left(1-\tilde{\sigma}_{0}\right)}\left(\frac{\Delta}{\tilde{z}}+\frac{1}{\tilde{z}^{2}}\right) \\
& \ll(u \log u)^{2}\left(\frac{\Delta}{z}+\frac{1}{z^{2}}\right) \ll \frac{u \mathscr{L}^{2}}{z^{\varepsilon}}\left(\Delta+\frac{1}{z}\right)
\end{aligned}
$$

by Proposition 3.1 and the hypothesis $u \leq z^{1-\varepsilon}$, and the first can be bounded, using (6.8), by

$$
\begin{aligned}
& \ll \tilde{z}^{-\tilde{\sigma}_{0}} \sum_{z \leq p<\tilde{z}} \log (\tilde{z} / z) \ll \tilde{z}^{1-\tilde{\sigma}_{0}} \frac{\tilde{z}-z+1}{\tilde{z}} \log (\tilde{z} / z) \\
& \ll(u \log u)\left(\Delta+\frac{1}{z}\right) \Delta \ll \frac{\Delta u \log u}{\mathscr{L}^{3}}+\frac{\Delta u \log u}{z} \ll \frac{\Delta u}{\mathscr{L}^{2}} .
\end{aligned}
$$

Moreover, since by Lemma 5.1 and (3.9),

$$
\begin{aligned}
\tilde{z}^{1-\tilde{s}_{0}} & =\frac{\tilde{z}^{1-\tilde{s}_{0}}}{1-\tilde{s}_{0}}\left(1-\tilde{s}_{0}\right) \\
& =\left(1+O\left(1 / \mathscr{L}^{2}\right)\right) \varphi^{\prime}\left(\tilde{z} ; \tilde{s}_{0}\right)\left(1-\tilde{s}_{0}\right)+O\left(\left|1-\tilde{s}_{0}\right|\right) \\
& =-\left(1+O\left(1 / \mathscr{L}^{2}\right)+O(1 / u \mathscr{L})\right) u\left(1-\tilde{s}_{0}\right)(\log \tilde{z})
\end{aligned}
$$


we have

$$
\begin{aligned}
\frac{1}{\tilde{z}^{\tilde{s}_{0}} \log \tilde{z}} \sum_{z \leq p<\tilde{z}} \log p \\
=\frac{\tilde{z}-z+E(\tilde{z}, z)}{\tilde{z}^{\tilde{s}_{0}} \log \tilde{z}} \\
=-\left(1+O\left(\frac{1}{\mathscr{L}^{2}}\right)+O\left(\frac{1}{u \mathscr{L}}\right)\right) u\left(1-\tilde{s}_{0}\right)\left(\frac{\tilde{z}-z}{\tilde{z}}+\frac{E(\tilde{z}, z)}{\tilde{z}}\right) \\
=-u\left(1-\tilde{s}_{0}\right)\left(\log \frac{\tilde{z}}{z}+\frac{E(\tilde{z}, z)}{\tilde{z}}\right)+O\left(\frac{u \Delta}{\mathscr{L}^{2}}\right)+O\left(u \Delta^{2}\right) \\
\quad+O\left(\frac{\left|1-\tilde{s}_{0}\right| \Delta}{\mathscr{L}}\right)+O\left(\frac{u}{z^{1-\varepsilon}}\right) .
\end{aligned}
$$

Inserting these estimates into (6.12), we obtain

$$
\left|R_{2}\right| \ll \frac{u}{z}|E(\tilde{z}, z)|+\frac{u \Delta}{\mathscr{L}^{2}}+u \Delta^{2}+\frac{\left|1-\tilde{s}_{0}\right| \Delta}{\mathscr{L}}+\frac{u}{z^{1-\varepsilon}},
$$

which proves the desired bound (6.6) for $R_{2}$ in view of (6.8), and the fact that $\left|1-\tilde{s}_{0}\right| \ll \xi_{u} / \mathscr{L} \ll \log u / \mathscr{L}$ by Proposition 3.1 and (3.2).

Thus the proof of Proposition 3.2 is complete.

\section{Proof of Theorem C: PRELIMINARIES}

In this section we derive estimates for the functions $F(z ; s)$ and $\varphi(z ; s)$ that are needed in the proof of Theorem C. We first state two auxiliary results from prime number theory.

Lemma 7.1. Let $0<\varepsilon \leq 1$ be fixed. Then we have, uniformly for $z \geq 2$, $0 \leq \sigma<1$, and $|t| \leq \exp \left((\log z)^{3 / 2-\varepsilon}\right)$,

$$
\sum_{n<z} \frac{\Lambda(n)}{n^{s}}=\frac{z^{1-s}-1}{1-s}+O\left(\frac{z^{1-\sigma}}{1-\sigma} \exp \left(-(\log z)^{\varepsilon / 2}\right)\right)+O(1) .
$$

Proof. This result is stated, in a slightly weaker form, in [HT, Lemma 6]. It can be established in a standard manner by writing the left-hand side as a Perron integral over the function $\zeta^{\prime} / \zeta$ and then shifting the line of integration into the critical strip, using Vinogradov's zero-free region. We omit the details.

Lemma 7.2. For any fixed $\varepsilon>0$ and uniformly for $\sigma \geq \varepsilon,|t| \geq 1$, we have

$$
\begin{gathered}
|\zeta(\sigma+i t)| \ll \max \left(1,|t|^{1-\sigma}\right) \log (1+|t|), \\
\left|\zeta^{\prime}(\sigma+i t)\right| \ll \max \left(1,|t|^{1-\sigma}\right) \log ^{2}(1+|t|) .
\end{gathered}
$$

Proof. The first estimate is well known; see, e.g., [Iv, Theorem 1.9]. The second estimate follows from the first by Cauchy's integral formula.

For the remainder of this section we let $z \geq 2$ be fixed and write $F(s)=$ $F(z ; s), \varphi(s)=\varphi(z ; s)$, and $\mathscr{L}=\log z$. The next three lemmas give upper bounds for these functions for various ranges of values $s$. 
Lemma 7.3. Let $0<\varepsilon \leq 1$ be fixed. Then we have, uniformly for $\sigma \geq \varepsilon$ and $|t| \geq 1$,

$$
|F(s)| \ll \begin{cases}\mathscr{L}|t|^{1 / \mathscr{L}} \log (1+|t|) & \text { if } \sigma \geq 1-1 / \mathscr{L}, \\ \mathscr{L}|t|^{1-\sigma} \log (1+|t|) \exp \left(2 z^{1-\sigma} /(1-\sigma) \mathscr{L}\right) & \text { if } \varepsilon \leq \sigma<1-1 / \mathscr{L} .\end{cases}
$$

Proof. Lemma 7.2 yields

$$
\begin{aligned}
|F(s)| & =\left|\zeta(s) \prod_{p<z}\left(1-\frac{1}{p^{s}}\right)\right| \\
& \leq|\zeta(s)| \prod_{p<z}\left(1+\frac{1}{p^{\sigma}}\right) \\
& \ll \max \left(1,|t|^{1-\sigma}\right) \log (1+|t|) \prod_{p<z}\left(1+\frac{1}{p^{\sigma}}\right) .
\end{aligned}
$$

In the case $\sigma \geq 1-1 / \mathscr{L}$, the desired bound follows from the estimate

$$
\begin{aligned}
\prod_{p<z}\left(1+\frac{1}{p^{\sigma}}\right) & \leq \exp \left\{\sum_{p<z} \frac{1}{p^{1-1 / \mathscr{L}}}\right\} \\
& =\exp \left\{\sum_{p<z} \frac{1}{p}+O(1)\right\} \ll \mathscr{L} .
\end{aligned}
$$

In the remaining case $\varepsilon \leq \sigma \leq 1-1 / \mathscr{L}$, we obtain the result from the estimates

$$
\begin{aligned}
\prod_{p<z}\left(1+\frac{1}{p^{\sigma}}\right) \ll\left|\zeta(\sigma)(1-\sigma) \prod_{p<z}\left(1-\frac{1}{p^{\sigma}}\right)\right|^{-1} & =\frac{\exp (-\operatorname{Re} \varphi(\sigma))}{1-\sigma} \\
& \leq \mathscr{L} \exp (|\varphi(\sigma)|)
\end{aligned}
$$

and (cf. Lemma 5.1)

$$
\begin{aligned}
|\varphi(\sigma)| & =\left(1+O\left(\frac{1}{\mathscr{L}}\right)\right) \int_{1}^{(1-\sigma) \mathscr{L}} \frac{e^{w}}{w} d w+O(1) \\
& \leq\left(1+O\left(\frac{1}{\mathscr{L}}\right)\right) \frac{z^{1-\sigma}}{(1-\sigma) \mathscr{L}}+O\left(\frac{z^{1-\sigma}}{(1-\sigma)^{2} \mathscr{L}^{2}}\right)+O(1) \\
& \leq \frac{2 z^{1-\sigma}}{(1-\sigma) \mathscr{L}}+O(1) .
\end{aligned}
$$

Lemma 7.4. Let $0<\varepsilon \leq 1$ be fixed. Uniformly in the range

$$
\varepsilon \leq \sigma \leq 1-1 / \mathscr{L}, \quad 1 \leq|t| \leq \exp \left(\mathscr{L}^{3 / 2-\varepsilon}\right),
$$

we have

$$
\begin{gathered}
|F(s)| \ll|t|^{1-\sigma} \log ^{2}(1+|t|) \exp \left((1+\varepsilon) z^{1-\sigma} / \mathscr{L}\right), \\
\left|F^{\prime}(s)\right| \ll|t|^{1-\sigma} \log ^{3}(1+|t|) z^{1-\sigma} \exp \left((1+\varepsilon) z^{1-\sigma} / \mathscr{L}\right) .
\end{gathered}
$$


Proof. Setting

$$
\psi(s)=\sum_{p<z} \log \left(1-\frac{1}{p^{s}}\right),
$$

we have, by Lemma 7.2, in the range (7.1)

$$
|F(s)|=|\zeta(s)| \exp (\operatorname{Re} \psi(s)) \ll|t|^{1-\sigma} \log (1+|t|) \exp (|\psi(s)|) .
$$

Thus, to prove (7.2) it suffices to show that

$$
|\psi(\sigma+i t)| \leq(1+\varepsilon) z^{1-\sigma} / \mathscr{L}+\log _{2}(1+|t|)+O(1)
$$

holds in this range. Similarly, since

$$
\begin{aligned}
\left|F^{\prime}(s)\right| & =\left|\zeta^{\prime}(s) \prod_{p<z}\left(1-\frac{1}{p^{s}}\right)+\zeta(s) \prod_{p<z}\left(1-\frac{1}{p^{s}}\right) \sum_{p<z} \frac{\log p}{p^{s}-1}\right| \\
& \ll|t|^{1-\sigma} \log ^{2}(1+|t|) \exp (|\psi(s)|)\left(1+\left|\sum_{p<z} \frac{\log p}{p^{s}-1}\right|\right)
\end{aligned}
$$

by Lemma $7.2,(7.3)$ is seen to be a consequence of (7.4) and the bound

$$
\left|\sum_{p<z} \frac{\log p}{p^{s}-1}\right| \ll z^{1-\sigma}
$$

Lemma 7.1 yields for the range (7.1)

$$
\begin{aligned}
\sum_{p<z} \frac{\log p}{p^{s}-1} & =\sum_{n<z} \frac{\Lambda(n)}{n^{2}}+O\left(\sum_{p<z} \frac{\log p}{p^{2 \sigma}}\right)+O(1) \\
& =\frac{z^{1-s}-1}{1-s}+O\left(\frac{z^{1-\sigma}}{1-\sigma} \exp \left(-\mathscr{L}^{z / 2}\right)\right)+O(1) .
\end{aligned}
$$

Since $|1-s| \geq|t| \geq 1$ and $1-\sigma \geq 1 / \mathscr{L}$ by (7.1), this gives (7.5). The same estimate shows that for $\sigma \leq \sigma^{\prime} \leq 1-1 / \mathscr{L}$, we have

$$
\left|\psi^{\prime}\left(\sigma^{\prime}+i t\right)\right|=\left|\sum_{p<z} \frac{\log p}{p^{\sigma^{\prime}+i t}-1}\right| \leq\left(1+O\left(\frac{1}{\mathscr{L}}\right)\right) z^{1-\sigma^{\prime}}+O(1) .
$$

This implies that

$$
\begin{aligned}
\left|\psi(\sigma+i t)-\psi\left(1-\frac{1}{\mathscr{L}}+i t\right)\right| & \leq\left(1+O\left(\frac{1}{\mathscr{L}}\right)\right) \int_{\sigma}^{1} z^{1-\sigma^{\prime}} d \sigma^{\prime}+O(1) \\
& \leq\left(1+O\left(\frac{1}{\mathscr{L}}\right)\right) \frac{z^{1-\sigma}}{\mathscr{L}}+O(1) \\
& \leq(1+\varepsilon) \frac{z^{1-\sigma}}{\mathscr{L}}+O(1)
\end{aligned}
$$

and since

$$
\begin{aligned}
\left|\psi\left(1-\frac{1}{\mathscr{L}}+i t\right)\right| & =\left|\sum_{p<z} \frac{1}{p^{1-1 / \mathscr{L}+i t}}\right|+O(1)=\left|\sum_{p} \frac{1}{p^{1+1 / \mathscr{L}+i t}}\right|+O(1) \\
& =\left|\log \zeta\left(1+\frac{1}{\mathscr{L}}+i t\right)\right|+O(1) \leq \log _{2}(1+|t|)+O(1)
\end{aligned}
$$

by Lemma 7.2, (7.4) follows. 
For the last lemma of this section we let $\varepsilon$ be a given positive number; $y$ and $z$ fixed numbers in the range $\left(R_{\varepsilon}\right)$; and $u=\log y / \log z$. We further let $s_{0}=s_{0}(y, z)=\sigma_{0}+i t_{0}$ be defined as in the theorem by (3.9) and (3.10). The implied constants are allowed to depend at most on $\varepsilon$.

Lemma 7.5. For $s=\sigma_{0}+i t,|t| \leq 1,|| t\left|-t_{0}\right|>1 / \mathscr{L}$, we have

$$
|F(s)| \ll\left|F\left(s_{0}\right)\right| \exp \left(-u / 3 \log ^{2} u\right) .
$$

Moreover, we have

$$
\left|F\left(s_{0}\right)\right|=\exp \{u(1+O(1 / \log u))\}
$$

Proof. Lemma 5.1 yields, for $s=\sigma+i t$ as in the lemma,

$$
F(s)=\exp (\varphi(s))=\exp \left\{-\operatorname{Ei}((1-s) \mathscr{L})+O\left(\frac{z^{1-\sigma}}{\mathscr{L}^{4}}\right)+O(1)\right\}
$$

where

$$
\operatorname{Ei}(w)=\int_{1}^{w} \frac{e^{v}}{v} d v
$$

is a variant of the exponential integral. By Proposition 3.1 and (3.2) the first error term can be bounded by

$$
\frac{z^{1-\sigma_{0}}}{\mathscr{L}^{4}} \ll \frac{e^{\xi_{u}}}{\mathscr{L}^{4}} \ll \frac{u \log u}{\mathscr{L}^{4}} \ll \frac{u}{\log ^{3} u} .
$$

Thus, the estimates (7.6) and (7.7) are seen to be equivalent to

$$
\operatorname{Re}\left\{\operatorname{Ei}\left(\left(1-s_{0}\right) \mathscr{L}\right)-\operatorname{Ei}((1-s) \mathscr{L})\right\} \leq-u / 3 \log ^{2} u+O(1)
$$

and

$$
\operatorname{Re}\left(\operatorname{Ei}\left(1-s_{0}\right) \mathscr{L}\right)=-u(1+O(1 / \log u)),
$$

respectively, and it suffices to prove the last two estimates.

We use the easily derived expansion

$$
\operatorname{Ei}(w)=e^{w}\left(\frac{1}{w}+\frac{1}{w^{2}}+\frac{2}{w^{3}}+O\left(\frac{1}{|\operatorname{Re} w|^{4}}\right)\right),
$$

which holds uniformly in $\operatorname{Re} w \geq 1$. Applying (7.10) with $w=\left(1-s_{0}\right) \mathscr{L}$ gives

$$
\begin{aligned}
& \operatorname{Ei}\left(\left(1-s_{0}\right) \mathscr{L}\right) \\
& \quad=\frac{z^{1-s_{0}}}{\left(1-s_{0}\right) \mathscr{L}}\left(1+\frac{1}{\left(1-s_{0}\right) \mathscr{L}}+\frac{2}{\left(1-s_{0}\right)^{2} \mathscr{L}^{2}}+O\left(\frac{\left|\left(1-s_{0}\right) \mathscr{L}\right|}{\left(1-\sigma_{0}\right)^{4} \mathscr{L}^{4}}\right)\right) .
\end{aligned}
$$


Now,

$$
\begin{aligned}
\frac{z^{1-s_{0}}}{\left(1-s_{0}\right) \mathscr{L}} & =\left(1+O\left(\frac{1}{\mathscr{L}^{3}}\right)\right) \frac{\varphi^{\prime}\left(s_{0}\right)}{\mathscr{L}}+O\left(\frac{1}{\mathscr{L}}\right) \\
& =-\left(1+O\left(\frac{1}{\mathscr{L}^{3}}\right)\right) u+O\left(\frac{1}{\mathscr{L}}\right)=-\left(1+O\left(\frac{1}{\log ^{3} u}\right)\right) u
\end{aligned}
$$

by Lemma 5.1 and (3.9), and since

$$
\left|\left(1-s_{0}\right) \mathscr{L}\right| \asymp\left(1-\sigma_{0}\right) \mathscr{L} \asymp \xi_{u} \asymp \log u
$$

by Proposition 3.1, we see that (7.11) implies (7.9). A further application of Proposition 3.1 shows that

$$
\begin{aligned}
\operatorname{Re} \frac{1}{\left(1-s_{0}\right) \mathscr{L}} & =\operatorname{Re}\left\{\frac{1}{\left(1-\sigma_{0}\right) \mathscr{L}}\left(1+i \frac{t_{0} \mathscr{L}}{\left(1-\sigma_{0}\right) \mathscr{L}}\right)+O\left(\frac{\left(t_{0} \mathscr{L}\right)^{2}}{\left(1-\sigma_{0}\right)^{3} \mathscr{L}^{3}}\right)\right\} \\
& =\frac{1}{\left(1-\sigma_{0}\right) \mathscr{L}}+O\left(\frac{1}{\log ^{3} u}\right)=\frac{1}{\xi_{u}}+O\left(\frac{1}{\log ^{3} u}\right)
\end{aligned}
$$

and

$$
\operatorname{Re} \frac{1}{\left(1-s_{0}\right)^{2} \mathscr{L}^{2}}=\frac{1}{\left(1-\sigma_{0}\right)^{2} \mathscr{L}^{2}}+O\left(\frac{1}{\log ^{3} u}\right)=\frac{1}{\xi_{u}^{2}}+O\left(\frac{1}{\log ^{3} u}\right)
$$

Therefore, from (7.11) and (7.12) we obtain the estimate:

$$
\operatorname{Re}\left(\operatorname{Ei}\left(\left(1-s_{0}\right) \mathscr{L}\right)\right)=-u\left(1+\frac{1}{\xi_{u}}+\frac{2}{\xi_{u}^{2}}+O\left(\frac{1}{\log ^{3} u}\right)\right)
$$

Next we apply (7.10) with $w=\left(1-\sigma_{0}-i t\right) \mathscr{L}$, where $|t| \leq 1$. Estimating each term on the right-hand side by its modulus in the same way as before, we obtain

$$
\begin{aligned}
|\operatorname{Ei}((1-s) \mathscr{L})| & \leq \frac{z^{1-\sigma_{0}}}{|1-s| \mathscr{L}}\left(1+\frac{1}{\xi_{u}}+\frac{2}{\xi_{u}^{2}}\right)+O\left(\frac{z^{1-\sigma_{0}}}{\log ^{4} u}\right) \\
& =\frac{\left|1-s_{0}\right| u}{|1-s|}\left(1+\frac{1}{\xi_{u}}+\frac{2}{\xi_{u}^{2}}\right)+O\left(\frac{u}{\log ^{3} u}\right) .
\end{aligned}
$$

Now if $1 \geq|t| \geq t_{0}+1 / \mathscr{L}$, then

$$
\begin{aligned}
\frac{\left|1-s_{0}\right|}{|1-s|} & =\left(\frac{\left(1-\sigma_{0}\right)^{2}+t_{0}^{2}}{\left(1-\sigma_{0}\right)^{2}+t^{2}}\right)^{1 / 2} \leq\left(1+\frac{1}{\left(\left(1-\sigma_{0}\right)^{2}+t_{0}^{2}\right) \mathscr{L}^{2}}\right)^{-1 / 2} \\
& =1-\frac{1}{2\left(\left(1-\sigma_{0}\right)^{2}+t_{0}^{2}\right) \mathscr{L}^{2}}+O\left(\frac{1}{\mathscr{L}^{4}\left(1-\sigma_{0}\right)^{4}}\right) \\
& =1-\frac{1}{2 \xi_{u}^{2}}+O\left(\frac{1}{\log ^{3} u}\right)
\end{aligned}
$$


Thus the comparison of (7.13) with (7.14) shows that

$$
\operatorname{Re}\left(\operatorname{Ei}\left(\left(1-s_{0}\right) \mathscr{L}\right)\right)+|\operatorname{Ei}((1-s) \mathscr{L})| \leq-\frac{u}{2 \xi_{u}^{2}}+O\left(\frac{u}{\log ^{3} u}\right),
$$

and hence (7.8), holds in the range $t_{0}+1 / \mathscr{L} \leq|t| \leq 1$.

Finally, to prove (7.8) for $|t| \leq t_{0}-1 / \mathscr{L}$, we apply $(7.10)$ with $w=(1-s) \mathscr{L}$ to obtain

$$
\begin{aligned}
\operatorname{Re}(\operatorname{Ei}((1-s) \mathscr{L})) & =\operatorname{Re} \frac{z^{1-s}}{(1-s) \mathscr{L}}+O\left(\frac{z^{1-\sigma_{0}}}{\left(1-\sigma_{0}\right)^{2} \mathscr{L}^{2}}\right) \\
& =\frac{z^{1-\sigma_{0}}}{\left(1-\sigma_{0}\right) \mathscr{L}} \cos (t \mathscr{L})+O\left(\frac{u}{\log u}\right) \\
& =u\left(\cos (t \mathscr{L})+O\left(\frac{1}{\log u}\right)\right) .
\end{aligned}
$$

Since $-\cos (t \mathscr{L}) \leq 0.9+O(1 / \log u)$ for $|t| \leq t_{0}-1 / \mathscr{L}=(\pi-1+O(1 / \log u)) / \mathscr{L}$, this implies that

$$
-\operatorname{Re}(\operatorname{Ei}((1-s) \mathscr{L})) \leq \frac{9}{10} u\left(1+O\left(\frac{1}{\log u}\right)\right)
$$

which, in conjunction with (7.13), proves (7.8) for the range $|t| \leq t_{0}-1 / \mathscr{L}$.

\section{Proof of Theorem C: Completion}

We begin by expressing $r(y, z)$ and $r(y, z ; \lambda)$ as Perron integrals over the functions $F(s)=F(z ; s)$.

Lemma 8.1. For any fixed $\varepsilon>0$ and uniformly for $y>0, z \geq z_{0}, \varepsilon<\sigma<1$, and $T \geq 1$, we have

$$
\begin{aligned}
r(y, z) V(z)= & \frac{1}{2 \pi i} \int_{\sigma-i T}^{\sigma+i T} \frac{F(s) y^{s-1}}{s} d s+O\left(\frac{\log \bar{y}}{T}\right) \\
& +O\left(\frac{\log \bar{y}}{y}\right)+O\left(\left(1+\frac{1}{y}\right) \frac{\mathscr{L}}{T^{\varepsilon / 2}} \exp \left(\frac{2 z^{1-\sigma}}{(1-\sigma) \mathscr{L}}\right)\right),
\end{aligned}
$$

where $V(z)=\prod_{p<z}(1-1 / p), \bar{y}=\max (y, 3), \mathscr{L}=\log z$, and the implied constants depend at most on $\varepsilon$.

Proof. Since the right-hand side of (8.1) is a continuous function of $y$, and since

$$
r(y, z) V(z)=\frac{1}{y} \Phi(y, z)-V(z)
$$

as a function of $y$, has jumps of order at most $O(1 / y)$, it suffices to prove (8.1) in the case where $y$ is not an integer. Perron's formula then gives, for any $\sigma_{1}>1$,

$$
\Phi(y, z)=\frac{1}{2 \pi i} \int_{\sigma_{1}-i T}^{\sigma_{1}+i T} \frac{F(s) y^{s}}{s} d s+O\left(\sum_{n=1}^{\infty}\left(\frac{y}{n}\right)^{\sigma_{1}} \min \left(1, \frac{1}{T|\log (y / n)|}\right)\right) .
$$


Taking $\sigma_{1}=1+1 / \log \bar{y}$, the error term becomes

$$
\begin{aligned}
& \ll \frac{y+1}{T} \sum_{n=1}^{\infty} \frac{1}{n^{\sigma_{1}}}+\sum_{y / 2<n<2 y} \min \left(1, \frac{y}{T|y-n|}\right) \\
& \ll \frac{y+1}{T\left(\sigma_{1}-1\right)}+\frac{y \log \bar{y}}{T}+1 \ll \frac{y \log \bar{y}}{T}+\log \bar{y} .
\end{aligned}
$$

Replacing the line of integration by the line $[\sigma-i T, \sigma+i T]$ introduces a residue $y V(z)$ from the pole of $F(s)=\zeta(s) \prod_{p<z}\left(1-1 / p^{s}\right)$ at $s=1$, and an error term that is of order

$$
\begin{aligned}
& \ll\left(\int_{\sigma-i T}^{\sigma_{1}-i T}+\int_{\sigma+i T}^{\sigma_{1}+i T}\right)\left|\frac{F(s) y^{s}}{s}\right||d s| \\
& \ll \frac{y+1}{T} \max _{\sigma^{\prime} \geq \sigma}\left(\left|F\left(\sigma^{\prime}+i T\right)\right|+\left|F\left(\sigma^{\prime}-i T\right)\right|\right) \\
& \ll \frac{y+1}{T} T^{1-\sigma+1 / \mathscr{L}} \log (1+T) \mathscr{L} \exp \left(\frac{2 z^{1-\sigma}}{(1-\sigma) \mathscr{L}}\right) \\
& \ll \frac{y+1}{T^{\varepsilon / 2}} \mathscr{L} \exp \left(\frac{2 z^{1-\sigma}}{(1-\sigma) \mathscr{L}}\right)
\end{aligned}
$$

by Lemma 7.3. Thus we have

$$
\begin{aligned}
y r(y, z) V(z)= & \varphi(y, z)-y V(z) \\
= & \frac{1}{2 \pi i} \int_{\sigma-i T}^{\sigma+i T} \frac{F(s) y^{s}}{s} d s+O\left(\frac{y \log \bar{y}}{T}\right)+O(\log \bar{y}) \\
& +O\left(\frac{y+1}{T^{\varepsilon / 2}} \mathscr{L} \exp \left(\frac{2 z^{1-\sigma}}{(1-\sigma) \mathscr{L}}\right)\right),
\end{aligned}
$$

from which (8.1) follows.

For the remainder of this section, we fix a positive number $\varepsilon$, which we can assume to be sufficiently small, and real numbers $y$ and $z$ in the range $R_{\varepsilon}$, and we let $s_{0}=\sigma_{0}+i t_{0}$ be defined by (3.9) and (3.10). Thus, in particular, we may assume that $y, z$, and $u=\log y / \log z$, are larger than any given constant. We also fix a real number $\lambda$ in the range

$$
2 \leq \lambda \leq \exp \left(\mathscr{L}^{3 / 2-\varepsilon}\right)
$$

and define the function

$$
k(s)=k_{\lambda}(s)=\exp \left(s^{2} / 2 \lambda\right) .
$$

In the sequel, the constants implied in the symbols " $O$ " and " $\ll$ " are allowed to depend on $\varepsilon$, but not on $y, z$, or $\lambda$.

Lemma 8.2. With $T_{0}=y^{3 / \varepsilon}$ we have

$$
r(y, z ; \lambda) V(z)=\frac{1}{2 \pi i} \int_{\sigma_{0}-i T_{0}}^{\sigma_{0}+i T_{0}} \frac{F(s) y^{s-1}}{s} k(s-1) d s+O\left(\frac{\log y}{y}\right) .
$$


Proof. Applying (8.1) with $\sigma=\sigma_{0}, T=T_{0}$, and $y e^{v}$ in place of $y$, we obtain

$$
\begin{aligned}
r(y, z ; \lambda) V(z) & =\frac{\sqrt{\lambda}}{\sqrt{2 \pi}} \int_{-\infty}^{\infty} r\left(y e^{v}, z\right) V(z) e^{-\lambda v^{2} / 2} d v \\
& =\frac{1}{2 \pi i} \int_{\sigma_{0}-i T_{0}}^{\sigma_{0}+i T_{0}} \frac{F(s) y^{s-1}}{s} k(s-1) d s+O\left(\sum_{i=1}^{3} R_{i}\right),
\end{aligned}
$$

where

$$
k(s-1)=\frac{\sqrt{\lambda}}{\sqrt{2 \pi}} \int_{-\infty}^{\infty} e^{v(s-1)-\lambda v^{2} / 2} d v=e^{(s-1)^{2} / 2 \lambda}
$$

is defined as in the lemma by (8.3),

$$
\begin{aligned}
R_{1} & =\sqrt{\lambda} \int_{-\infty}^{\infty} \frac{\log \left(\overline{y e^{v}}\right)}{T_{0}} e^{-\lambda v^{2} / 2} d v \\
& \ll \sqrt{\lambda} \int_{-\infty}^{\infty} \frac{\log y+e^{v}}{T_{0}} e^{-\lambda v^{2} / 2} d v \ll \frac{\log y}{y^{3 / \varepsilon}}, \\
R_{2} & =\sqrt{\lambda} \int_{-\infty}^{\infty} \frac{\log \left(\overline{y e^{v}}\right)}{y e^{v}} e^{-\lambda v^{2} / 2} d v \\
& \ll \sqrt{\lambda} \frac{\log y}{y} \int_{-\infty}^{\infty}\left(1+e^{|v|}\right) e^{-\lambda v^{2} / 2} d v \ll \frac{\log y}{y},
\end{aligned}
$$

and

$$
\begin{aligned}
R_{3} & =\frac{\sqrt{\lambda}}{T_{0}^{\varepsilon / 2}} \mathscr{L} \exp \left(\frac{2 z^{1-\sigma_{0}}}{\left(1-\sigma_{0}\right) \mathscr{L}}\right) \int_{-\infty}^{\infty}\left(1+\frac{1}{y e^{v}}\right) e^{-\lambda v^{2} / 2} d v \\
& \ll \frac{\mathscr{L}}{y^{3 / 2}} \exp \left(\frac{2 z^{1-\sigma_{0}}}{\left(1-\sigma_{0}\right) \mathscr{L}}\right) .
\end{aligned}
$$

The error terms $R_{1}$ and $R_{2}$ are clearly of order $O(\log y / y$ ) (assuming, as we may, that $\varepsilon \leq 2$ ), and the same holds for the term $R_{3}$, since $\mathscr{L}=\log z \ll$ $\log y$, and by Proposition 3.1,

$$
\frac{z^{1-\sigma_{0}}}{\left(1-\sigma_{0}\right) \mathscr{L}} \ll u=\frac{\log y}{\mathscr{L}} .
$$

This proves (8.4).

Lemma 8.3. We have

$$
\begin{aligned}
r(y, z ; \lambda) V(z)= & \frac{1}{2 \pi i} \int_{\sigma_{0}-i}^{\sigma_{0}+i} \frac{F(s) y^{s-1}}{s} k(s-1) d s \\
& +O\left(\frac{y^{\sigma_{0}-1} e^{(1-\varepsilon / 3) u} \lambda^{1-\sigma_{0}}(\log \lambda)^{4}}{\mathscr{L}}\right) .
\end{aligned}
$$

Proof. In view of the previous lemma, and since

$$
\frac{\log y}{y} \ll \frac{y^{\varepsilon / 2-1}}{\mathscr{L}} \leq \frac{y^{\sigma_{0}-1}}{\mathscr{L}},
$$


it suffices to show that the contribution of the range $1 \leq|t| \leq T_{0}$ to the integral in (8.4) is bounded by the error term in (8.5). We split this range into the two parts $1 \leq|t| \leq T_{1}$ and $T_{1}<|t| \leq T_{0}$, where

$$
T_{1}=\min \left(T_{0}, \exp \left(\mathscr{L}^{3 / 2-\varepsilon}\right)\right) \text {. }
$$

Since

$$
\left|k\left(\sigma_{0}-1+i t\right)\right|=\exp \left(\frac{\left(\sigma_{0}-1\right)^{2}-t^{2}}{2 \lambda}\right) \ll e^{-t^{2} / 2 \lambda}
$$

and

$$
\begin{aligned}
\max _{|t| \leq T_{0}}\left|F\left(\sigma_{0}+i t\right)\right| & \ll T_{0}^{1-\sigma_{0}} \log \left(1+T_{0}\right) \mathscr{L} \exp \left(\frac{2 z^{1-\sigma_{0}}}{\left(1-\sigma_{0}\right) \mathscr{L}}\right) \\
& \ll T_{0} \mathscr{L} \exp (O(u)) \ll y^{4 / \varepsilon}
\end{aligned}
$$

by Lemma 7.3 and Proposition 3.1, the integral over the range $T_{1}<|t| \leq T_{0}$ is bounded by

$$
\begin{aligned}
& \ll \max _{|t| \leq T_{0}}\left|F\left(\sigma_{0}+i t\right)\right| \int_{T_{1}}^{T_{0}}\left|k\left(\sigma_{0}-1+i t\right)\right| d t \\
& \ll y^{4 / \varepsilon} \int_{T_{1}}^{\infty} e^{-t^{2} / 2 \lambda} d t \ll y^{4 / \varepsilon} \sqrt{\lambda} e^{-T_{1}^{2} / 2 \lambda} .
\end{aligned}
$$

If $\lambda \leq T_{0}$, then we have $T_{1} \geq \lambda$ by (8.2) and the definition of $T_{1}$, and the above bound becomes

$$
\ll y^{4 / \varepsilon} \sqrt{\lambda} e^{-T_{1} / 2} \ll y^{6 / \varepsilon} e^{-z / 2} \ll 1 / y,
$$

since $T_{1} \geq \min (y, \exp (\mathscr{L}))=z$ and $z \gg(\log y)^{1 /(1-\varepsilon / 2)}$ in the range $\left(R_{\varepsilon}\right)$. On the other hand, if $\lambda>T_{0}$, then (8.2) implies $\exp \left(\mathscr{L}^{2 / 3-\varepsilon}\right)>T_{0}$, in which case $T_{1}=T_{0}$ and the range $T_{1}<|t| \leq T_{0}$ is empty. In either case, the contribution of this range is of the desired order, and to prove (8.5) it remains to show that the same is true for the range $1 \leq|t| \leq T_{1}$.

Since $F(\bar{s})=\overline{F(s)}$ and $k(\bar{s})=\overline{k(s)}$, it suffices to consider the integral

$$
I=\int_{\sigma_{0}+i}^{\sigma_{0}+i T_{1}} \frac{F(s) y^{s-1}}{s} k(s-1) d s .
$$

Now

where

$$
I=i y^{\sigma_{0}-1} I_{1}
$$

$$
I_{1}=\int_{1}^{T_{1}} G(t) y^{i t} d t
$$

with

$$
G(t)=\frac{F\left(\sigma_{0}+i t\right)}{\sigma_{0}+i t} k\left(\sigma_{0}-1+i t\right)
$$


Hence, in order to prove the desired estimate, it suffices to show that

$$
\left.\left|I_{1}\right| \ll \frac{1}{\mathscr{L}} \lambda^{1-\sigma_{0}}(\log \lambda)^{4} \exp \left\{\left(1-\frac{\varepsilon}{3}\right) u\right)\right\} .
$$

At this point a trivial bound of $I_{1}$ by the integral over the modulus of $G(t)$ would not be sufficient to yield (8.9). Therefore, we have to proceed more carefully, and we first apply an integration by parts to get

$$
I_{1}=\left.\frac{y^{i t}}{i \log y} G(t)\right|_{1} ^{T_{1}}-\frac{1}{i \log y} \int_{1}^{T_{1}} G^{\prime}(t) y^{i t} d t,
$$

which implies

$$
\left|I_{1}\right| \ll \frac{1}{\mathscr{L}}\left\{\max _{t=1, T_{1}}|G(t)|+\int_{1}^{T_{1}}\left|G^{\prime}(t)\right| d t\right\} .
$$

We estimate the terms on the right-hand side using Lemmas 7.4 and 7.5.

By Lemma 7.4 (with $\varepsilon / 3$ in place of $\varepsilon$ ) and Proposition 3.1 we have, for $1 \leq t \leq T_{1}$,

$$
\begin{aligned}
\left|F\left(\sigma_{0}+i t\right)\right| & \ll t^{1-\sigma_{0}} \log ^{2}(1+t) \exp \left\{\left(1+\frac{\varepsilon}{3}\right) \frac{z^{1-\sigma_{0}}}{\mathscr{L}}\right\} \\
& \ll t^{1-\sigma_{0}} \log ^{2}(1+t) \exp \left\{\left(1+\frac{\varepsilon}{3}\right) \frac{e^{\xi_{u}}}{\mathscr{L}}\left(1+O\left(\frac{1}{\log u}\right)\right)\right\} \\
& \ll t^{1-\sigma_{0}} \log ^{2}(1+t) \exp \left\{\left(1-\frac{\varepsilon}{3}\right) u\right\},
\end{aligned}
$$

where in the last estimate we have used the identity $e^{\xi_{u}}=u \xi_{u}+1$, the estimate (3.2) for $\xi_{u}$, and the bound $\log u \leq(1-\varepsilon) \mathscr{L}$. It follows that

$$
\begin{aligned}
\max _{t=1, T_{1}}|G(t)| & \ll \max _{t=1, T_{1}} \frac{\left|F\left(\sigma_{0}+i t\right)\right|}{t} e^{-t^{2} / 2 \lambda} \\
& \ll \exp \{(1-\varepsilon / 3) u\} .
\end{aligned}
$$

A similar argument shows that, for $1 \leq t \leq T_{1}$,

$$
\left|F^{\prime}\left(\sigma_{0}+i t\right)\right| \ll t^{1-\sigma_{0}} \log ^{3}(1+t) \exp \{(1-\varepsilon / 3) u\}
$$

and hence,

$$
\begin{aligned}
\left|G^{\prime}(t)\right| & \ll \frac{\left|F^{\prime}\left(\sigma_{0}+i t\right)\right| e^{-t^{2} / 2 \lambda}}{t}+\frac{\left|F\left(\sigma_{0}+i t\right)\right| e^{-t^{2} / 2 \lambda}}{t}\left(\frac{1}{t}+\frac{t}{\lambda}\right) \\
& \ll t^{-\sigma_{0}}(1+t / \lambda) e^{-t^{2} / 2 \lambda} \log ^{3}(1+t) \exp \{(1-\varepsilon / 3) u\} .
\end{aligned}
$$

It follows that

$$
\begin{aligned}
\int_{1}^{T_{1}}\left|G^{\prime}(t)\right| d t & \ll\left\{\int_{1}^{\lambda} \frac{d t}{t^{\sigma_{0}}}+\frac{1}{\lambda} \int_{\lambda}^{\infty} t e^{-t^{2} / 2 \lambda} d t\right\}(\log \lambda)^{3} \exp \left\{\left(1-\frac{\varepsilon}{3}\right) u\right\} \\
& \ll \lambda^{1-\sigma_{0}}(\log \lambda)^{4} \exp \{(1-\varepsilon / 3) u\} .
\end{aligned}
$$


Combining these estimates we obtain (8.9).

Lemma 8.4. With $\delta=1 / \mathscr{L}$ we have

$$
\begin{aligned}
r(y, z ; \lambda) V(z)= & \frac{1}{\pi} \operatorname{Re} \int_{t_{0}-\delta}^{t_{0}+\delta} \frac{F\left(\sigma_{0}+i t\right) y^{\sigma_{0}-1+i t}}{\sigma_{0}+i t} k\left(\sigma_{0}-1+i t\right) d t \\
& +O\left(\frac{y^{\sigma_{0}-1} e^{(1-\varepsilon / 3) u} \lambda^{1-\sigma_{0}}(\log \lambda)^{4}}{\mathscr{L}}\right) \\
& +O\left(\frac{y^{\sigma_{0}-1}\left|F\left(s_{0}\right)\right|}{\mathscr{L}} \exp \left(-\frac{u}{4 \log ^{2} u}\right)\right) .
\end{aligned}
$$

Proof. Since $F\left(\sigma_{0}-i t\right)=\overline{F\left(\sigma_{0}+i t\right)}$ and $k\left(\sigma_{0}-1-i t\right)=\overline{k\left(\sigma_{0}-1+i t\right)}$, the integrand in (8.10) changes into its complex conjugate when $t$ is replaced by $-t$. Thus, the main term on the right-hand side of $(8.10)$ can be written as

$$
\frac{1}{2 \pi i}\left(\int_{\sigma_{0}-i\left(t_{0}+\delta\right)}^{\sigma_{0}-i\left(t_{0}-\delta\right)}+\int_{\sigma_{0}+i\left(t_{0}-\delta\right)}^{\sigma_{0}+i\left(t_{0}+\delta\right)}\right) \frac{F(s) y^{s-1}}{s} k(s-1) d s .
$$

By Lemma 8.3, then, it suffices to show that the contribution of the ranges $|t| \leq t_{0}-\delta$ and $t_{0}+\delta \leq|t| \leq 1$ to the integral in (8.5) is of the order of the second error term in (8.10).

For the range $|t| \leq t_{0}-\delta$ the trivial estimate

$$
\left|\int_{\sigma_{0}-i\left(t_{0}-\delta\right)}^{\sigma_{0}+i\left(t_{0}-\delta\right)} \frac{F(s) y^{s-1}}{s} k(s-1)\right| \ll y^{\sigma_{0}-1} t_{0} \max _{|t| \leq t_{0}-\delta}\left|F\left(\sigma_{0}+i t\right)\right|
$$

is sufficient, since $t_{0} \ll 1 / \mathscr{L}$ by Proposition 3.1, and

$$
\max _{|t| \leq t_{0}-\delta}\left|F\left(\sigma_{0}+i t\right)\right| \ll\left|F\left(s_{0}\right)\right| \exp \left(-u / 3 \log ^{2} u\right)
$$

by Lemma 7.5. The second range $t_{0}+\delta \leq|t| \leq 1$ requires a more delicate argument. It suffices to consider the integral

$$
\begin{aligned}
I & =\int_{t_{0}+\delta}^{1} \frac{F\left(\sigma_{0}+i t\right) y^{\sigma_{0}-1+i t}}{\sigma_{0}+i t} k\left(\sigma_{0}-1+i t\right) d t \\
& =y^{\sigma_{0}-1} \int_{t_{0}+\delta}^{1} G(t) y^{i t} d t=y^{\sigma_{0}-1} I_{1},
\end{aligned}
$$

say, where $G(t)$ is defined by (8.8). We have to show that

$$
\left|I_{1}\right| \ll \frac{\left|F\left(s_{0}\right)\right|}{\mathscr{L}} \exp \left(-\frac{u}{4 \log ^{2} u}\right) .
$$

Partial integration gives

$$
I_{1} \ll \frac{1}{\log y}\left\{\max _{t_{0}+\delta \leq t \leq 1}|G(t)|+\left|\int_{t_{0}+\delta}^{1} G^{\prime}(t) y^{i t} d t\right|\right\} .
$$


By (8.8), (8.6), and Lemma 7.5, we have

$$
\max _{t_{0}+\delta \leq t \leq 1}|G(t)| \ll \max _{t_{0}+\delta \leq t \leq 1}\left|F\left(\sigma_{0}+i t\right)\right| \ll\left|F\left(s_{0}\right)\right| \exp \left(-\frac{u}{3 \log ^{2} u}\right) .
$$

Thus, in order to prove (8.11), it remains to show that the integral

$$
I_{2}=\int_{t_{0}+\delta}^{1} G^{\prime}(t) y^{i t} d t
$$

satisfies

$$
\left|I_{2}\right| \ll\left|F\left(s_{0}\right)\right| \exp \left(-u / 4 \log ^{2} u\right) .
$$

By (8.8), (8.6), Lemma 5.1, and Proposition 3.1, we have, for $|t| \leq 1$,

$$
\begin{aligned}
G^{\prime}(t) & =\frac{i F\left(\sigma_{0}+i t\right) k\left(\sigma_{0}-1+i t\right)}{\sigma_{0}+i t}\left\{\frac{F^{\prime}\left(\sigma_{0}+i t\right)}{F\left(\sigma_{0}+i t\right)}-\frac{1}{\sigma_{0}+i t}+\frac{k^{\prime}\left(\sigma_{0}-1+i t\right)}{k\left(\sigma_{0}-1+i t\right)}\right\} \\
& =i G(t)\left\{\phi^{\prime}\left(\sigma_{0}+i t\right)+O(1)\right\} \\
& =i G(t)\left\{\frac{z^{1-\sigma_{0}-i t}}{1-\sigma_{0}-i t}+O\left(\frac{z^{1-\sigma_{0}}}{\left(1-\sigma_{0}\right) \mathscr{L}}\right)+O(1)\right\} \\
& =G_{1}(t) z^{-i t}+O(u|G(t)|),
\end{aligned}
$$

where

$$
G_{1}(t)=\frac{i z^{1-\sigma_{0}}}{1-\sigma_{0}-i t} G(t)
$$

In view of the bound (8.12), the contribution of the O-term to the integral $I_{2}$ is of order

$$
\ll u \max _{t_{0}+\delta \leq t \leq 1}|G(t)| \ll u\left|F\left(s_{0}\right)\right| \exp \left(-u / 3 \log ^{2} u\right),
$$

which is sufficient. Moreover, a further integration by parts yields

$$
\left|\int_{t_{0}+\delta}^{1} G_{1}(t) z^{-i t} y^{i t} d t\right| \ll \frac{1}{\log (y / z)}\left\{\max _{t_{0}+\delta \leq t \leq 1}\left|G_{1}(t)\right|+\int_{t_{0}+\delta}^{1}\left|G_{1}^{\prime}(t)\right| d t\right\} .
$$

Since

$$
\log (y / z)=(u-1) \mathscr{L} \gg u \mathscr{L}
$$

if $u \geq 2$, as we may assume, and since

$$
\begin{aligned}
\left|G_{1}(t)\right| & \ll \frac{z^{1-\sigma_{0}}}{\left|1-\sigma_{0}-i t\right|}|G(t)| \ll \frac{u \log u}{|t|}|G(t)|, \\
\left|G_{1}^{\prime}(t)\right| & \ll \frac{z^{1-\sigma_{0}}}{\left|1-\sigma_{0}-i t\right|^{2}}|G(t)|+\frac{z^{1-\sigma_{0}}}{\left|1-\sigma_{0}-i t\right|}\left|G^{\prime}(t)\right| \\
& \ll \frac{z^{1-\sigma_{0}}}{t_{1}}|G(t)|+\frac{z^{1-\sigma_{0}}}{t}\left(\left|G_{1}(t)\right|+u|G(t)|\right) \\
& \ll \frac{u^{2} \log ^{2} u}{t^{2}}|G(t)|
\end{aligned}
$$


by (8.14) and Proposition 3.1, the right-hand side of (8.15) is bounded by

$$
\begin{aligned}
& \ll \frac{1}{\mathscr{L}} \max _{t_{0}+\delta \leq t \leq 1}|G(t)|\left\{\frac{u \log u}{t_{0}}+\int_{t_{0}+\delta}^{1} \frac{u^{2} \log ^{2} u}{t^{2}} d t\right\} \\
& \ll \frac{u^{2} \log ^{2} u}{t_{0} \mathscr{L}} \max _{t_{0}+\delta \leq t \leq 1}|G(t)| \\
& \ll u^{2}(\log u)^{2}\left|F\left(s_{0}\right)\right| \exp \left(-u / 3 \log ^{2} u\right),
\end{aligned}
$$

where the last estimate follows from (8.12) and the bound $t_{0} \gg 1 / \mathscr{L}$ (cf. (3.12)). This implies the desired bound (8.13) and completes the proof of Lemma 8.4.

Lemma 8.5. With $\delta=1 / \mathscr{L}$ we have

$$
\begin{aligned}
& \int_{t_{0}-\delta}^{t_{0}+\delta} \frac{F\left(\sigma_{0}+i t\right) y^{\sigma_{0}-1+i t}}{\sigma_{0}+i t} k\left(\sigma_{0}-1+i t\right) d t \\
& =\left(1+O\left(\frac{1}{\log u}\right)\right) \frac{y^{s_{0}-1} F\left(s_{0}\right) k\left(s_{0}-1\right)}{s_{0} \mathscr{L} \sqrt{u / 2 \pi}} .
\end{aligned}
$$

Proof. The integrand in (8.16) can be written as

$$
\left(1+O\left(\frac{1}{\mathscr{L}}\right)\right) \frac{y^{s_{0}-1} k\left(s_{0}-1\right)}{s_{0}} \exp \left\{i\left(t-t_{0}\right) \log y+\varphi\left(\sigma_{0}+i t\right)\right\},
$$

since $t=t_{0}+O(1 / \mathscr{L})=O(1 / \mathscr{L})$ and $k\left(\sigma_{0}-1+i t\right)=k\left(s_{0}-1\right)(1+O(1 / \mathscr{L}))$ in the range of integration. Expanding $\varphi\left(\sigma_{0}+i t\right)$ in a Taylor series about $t=t_{0}$, we obtain

$$
\begin{aligned}
i\left(t-t_{0}\right) \log y+\varphi\left(\sigma_{0}+i t\right)= & \varphi\left(s_{0}\right)+i\left(t-t_{0}\right)\left(\log y+\varphi^{\prime}\left(s_{0}\right)\right) \\
& -\frac{1}{2}\left(t-t_{0}\right)^{2} \varphi^{\prime \prime}\left(s_{0}\right)-\frac{i}{3 !}\left(\tau-t_{0}\right)^{3} \varphi^{\prime \prime \prime}\left(\sigma_{0}+i \tau\right)
\end{aligned}
$$

with a suitable $\tau=\tau(t),\left|\tau-t_{0}\right| \leq\left|t-t_{0}\right|$. By the definition of $s_{0}$, the linear term here vanishes. Moreover, from Lemma 5.1 and Proposition 3.1, we see that

$$
\begin{aligned}
\varphi^{\prime \prime}\left(s_{0}\right) & =\frac{-z^{1-s_{0}} \mathscr{L}}{1-s_{0}}\left(1+O\left(\frac{1}{\left(1-\sigma_{0}\right) \mathscr{L}}\right)\right) \\
& =u \mathscr{L}^{2}(1+O(1 / \log u))
\end{aligned}
$$

and

$$
\left|\varphi^{\prime \prime \prime}\left(\sigma_{0}+i \tau\right)\right| \leq \frac{z^{1-\sigma_{0}} \mathscr{L}^{2}}{1-\sigma_{0}}\left(1+O\left(\frac{1}{\left(1-\sigma_{0}\right) \mathscr{L}}\right)\right)=u \mathscr{L}^{3}\left(1+O\left(\frac{1}{\log u}\right)\right) .
$$

Thus we can write $(8.17)$ as

$$
(1+O(1 / \mathscr{L})) M \exp \left\{-\frac{1}{2}\left(t-t_{0}\right)^{2} u \mathscr{L}^{2}+R\left(t-t_{0}\right)\right\}
$$

where

$$
M=\frac{y^{s_{0}-1} e^{\varphi\left(s_{0}\right)} k\left(s_{0}-1\right)}{s_{0}}=\frac{y^{s_{0}-1} F\left(s_{0}\right) k\left(s_{0}-1\right)}{s_{0}}
$$


and the function $R(t)$ satisfies

$$
|R(t)| \leq \frac{1}{3 !} t^{3} \mathscr{L}^{3} u+O\left(t^{2} \mathscr{L}^{2} \frac{u}{\log u}\right)+O\left(t^{3} \mathscr{L}^{3} \frac{u}{\log u}\right) .
$$

Setting

$$
\delta_{1}=\delta\left(\frac{\log u}{u}\right)^{1 / 2}=\frac{1}{\mathscr{L}}\left(\frac{\log u}{u}\right)^{1 / 2},
$$

we have, for $\left|t-t_{0}\right| \leq \delta_{1}, R\left(t-t_{0}\right)=O(1)$, and

$$
\exp \left(R\left(t-t_{0}\right)\right)=1+O\left(R\left(t-t_{0}\right)\right)=1+O\left(\left(t-t_{0}\right)^{2} \mathscr{L}^{2} \frac{u}{\log u}\right)
$$

and for $\left|t-t_{0}\right| \leq \delta=1 / \mathscr{L}$,

$$
\begin{aligned}
\exp \{ & \left.-\frac{1}{2}\left(t-t_{0}\right)^{2} u \mathscr{L}^{2}+R\left(t-t_{0}\right)\right\} \\
& \leq \exp \left\{-\frac{1}{2}\left(t-t_{0}\right)^{2} u \mathscr{L}^{2}+\frac{1}{3 !}\left(t-t_{0}\right)^{2} u \mathscr{L}^{2}\left(1+O\left(\frac{1}{\log u}\right)\right)\right\} \\
& \ll \exp \left\{-\frac{1}{4}\left(t-t_{0}\right)^{2} u \mathscr{L}^{2}\right\} .
\end{aligned}
$$

Thus, the integral of (8.18) over $\left|t-t_{0}\right| \leq \delta$ equals

$$
\begin{aligned}
M \int_{t_{0}-\delta_{1}}^{t_{0}+\delta_{1}} & \left(1+O\left(\frac{1}{\mathscr{L}}\right)+O\left(\left(t-t_{0}\right)^{2} \mathscr{L}^{2} \frac{u}{\log u}\right)\right) e^{-\left(t-t_{0}\right)^{2} u \mathscr{L}^{2} / 2} d t \\
& +O\left(|M| \int_{t_{0}+\delta_{1}}^{t_{0}+\delta} e^{-\left(t-t_{0}\right)^{2} u \mathscr{L}^{2} / 4} d t\right) \\
= & \frac{M}{\sqrt{u} \mathscr{L}} \int_{-\delta_{1} \sqrt{u} \mathscr{L}}^{\delta_{1} \sqrt{u} \mathscr{L}}\left(1+O\left(\frac{1}{\mathscr{L}}\right)+O\left(\frac{\tau^{2}}{\log u}\right)\right) e^{-\tau^{2} / 2} d \tau \\
& +O\left(\frac{|M|}{\sqrt{u} \mathscr{L}} \int_{\delta_{1} \sqrt{u} \mathscr{L}}^{\infty} e^{-\tau^{2} / 4} d \tau\right) \\
= & \frac{M \sqrt{2 \pi}}{\sqrt{u} \mathscr{L}}\left(1+O\left(\frac{1}{\log u}\right)\right),
\end{aligned}
$$

where the last estimate follows since

$$
\int_{-\infty}^{\infty} e^{-\tau^{2} / 2} d \tau=\sqrt{2 \pi}
$$

and

$$
\int_{\delta_{1} \sqrt{u} \mathscr{L}}^{\infty} e^{-\tau^{2} / 4} d \tau=\int_{\sqrt{\log u}}^{\infty} e^{-\tau^{2} / 4} d \tau \ll e^{-(\log u) / 4} \ll \frac{1}{\log u} .
$$

This proves (8.16). 
Proof of Theorem $C$. We now suppose that $\lambda$ satisfies the hypothesis $\log u \leq$ $\lambda \leq \exp \left(\mathscr{L}^{3 / 2-\varepsilon}\right)$ of the theorem. Combining Lemmas 8.4 and 8.5 we obtain

$$
\begin{aligned}
r(y, z ; \lambda)= & \operatorname{Re} \frac{y^{s_{0}-1} F\left(s_{0}\right) k\left(s_{0}-1\right)}{\pi V(z) s_{0} \mathscr{L} \sqrt{u / 2 \pi}}+O\left(\frac{y^{\sigma_{0}-1}\left|F\left(s_{0}\right)\right|}{V(z) \mathscr{L} \sqrt{u} \log u}\right) \\
& +O\left(\frac{y^{\sigma_{0}-1} e^{(1-\varepsilon / 3) u} \lambda^{1-\sigma_{0}}(\log \lambda)^{4}}{V(z) \mathscr{L}}\right) \\
& +O\left(\frac{y^{\sigma_{0}-1}\left|F\left(s_{0}\right)\right|}{V(z) \mathscr{L}} \exp \left(-\frac{u}{4 \log ^{2} u}\right)\right) .
\end{aligned}
$$

Since

$$
k\left(s_{0}-1\right)=e^{\left(s_{0}-1\right)^{2} / 2 \lambda}=1+O\left(\frac{1}{\lambda}\right)=1+O\left(\frac{1}{\log u}\right)
$$

by the assumption $\lambda \geq \log u$ of Theorem $\mathrm{C}$, and since by Mertens' formula,

$$
V(z) \mathscr{L}=\prod_{p<z}\left(1-\frac{1}{p}\right) \log z=e^{-\gamma}\left(1+O\left(\frac{1}{\mathscr{L}}\right)\right),
$$

the main term in (8.19) is equal to

$$
\operatorname{Re} \frac{y^{s_{0}-1} F\left(s_{0}\right)}{\pi e^{-\gamma} s_{0} \sqrt{u / 2 \pi}}+O\left(\frac{y^{\sigma_{0}-1}\left|F\left(s_{0}\right)\right|}{\sqrt{u} \log u}\right)=\operatorname{Re} \rho(y, z)+O\left(\frac{|\rho(y, z)|}{\log u}\right) .
$$

The same argument shows that the first and third error terms are bounded by $O(|\rho(y, z)| / \log u)$. Finally, using the seconds part of Lemma 7.5 , the second error term is seen to be of order

$$
\begin{aligned}
& \ll y^{\sigma_{0}-1} e^{(1-\varepsilon / 3) u} \lambda^{1-\sigma_{0}}(\log \lambda)^{4} \\
& =y^{\sigma_{0}-1}\left|F\left(s_{0}\right)\right| \exp \left\{-\frac{\varepsilon}{3} u\left(1+O\left(\frac{1}{\log u}\right)\right)\right\} \lambda^{1-\sigma_{0}}(\log \lambda)^{4} \\
& \ll|\rho(y, z)| e^{-\varepsilon u / 4} \lambda^{1-\sigma_{0}}(\log \lambda)^{4} .
\end{aligned}
$$

Altogether, we obtain

$$
r(y, z ; \lambda)=\operatorname{Re} \rho(y, z)+O\left(\frac{|\rho(y, z)|}{\log u}\right)+O\left(|\rho(y, z)| e^{-\varepsilon u / 4} \lambda^{1-\sigma_{0}}(\log \lambda)^{4}\right) .
$$

This implies the desired estimate (3.11) in the case $\lambda$ satisfies, in addition to the hypotheses of the theorem, the inequalities $\lambda^{1-\sigma_{0}} \leq e^{\varepsilon u / 8}$ and $\log \lambda \leq u^{2}$. Therefore it remains to show that the same estimate holds for values $\lambda$ in the range

$$
\min \left(u^{2}, \frac{\varepsilon u}{8\left(1-\sigma_{0}\right)}\right) \leq \log \lambda \leq \mathscr{L}^{3 / 2-\varepsilon}
$$

Since $1-\sigma_{0}=\left(\xi_{u} / \mathscr{L}\right)\left(1+O\left(1 / \log ^{2} u\right)\right) \ll \log u / \mathscr{L}$ by Lemma 5.1 , the range (8.20) is only nonempty in the case $u \ll \mathscr{L}$, which we may henceforth assume. 
We shall show that

$$
r(y, z ; \lambda)=r(y, z)+O\left(\frac{1}{\sqrt{\lambda}}\right)+O\left(\frac{z}{y}\right)
$$

holds for any $\lambda \geq 1$. Now if $\lambda$ is in the range (8.20), then the error terms in (8.21) are bounded by

$$
\begin{aligned}
\frac{1}{\sqrt{\lambda}}+\frac{z}{y} & \ll \exp \left\{-\frac{\varepsilon u}{16\left(1-\sigma_{0}\right)}\right\}+e^{-u^{2} / 2}+e^{-(u-1) \mathscr{L}} \\
& \ll \exp \left\{-\frac{\varepsilon u \mathscr{L}}{16 \xi_{u}}\left(1+O\left(\frac{1}{\log u}\right)\right)\right\}+e^{-u^{2} / 2}+e^{-(u-1) \mathscr{L}} \\
& \ll \exp \left(-u^{3 / 2}\right) \ll \frac{|\rho(y, z)|}{\log u}
\end{aligned}
$$

in view of Propositions 3.2 and 3.2, and the bound $u \ll \mathscr{L}$. Thus, setting

$$
\lambda_{1}=\exp \left\{\min \left(u^{2}, \frac{\varepsilon u}{8\left(1-\sigma_{0}\right)}\right)\right\},
$$

we obtain

$$
\begin{aligned}
r(y, z ; \lambda) & =r\left(y, z ; \lambda_{1}\right)+O\left(\frac{|\rho(y, z)|}{\log u}\right) \\
& =\operatorname{Re} \rho(y, z)+O\left(\frac{|\rho(y, z)|}{\log u}\right)
\end{aligned}
$$

since (3.11) holds for $\lambda=\lambda_{1}$. This shows that the desired estimate (3.11) remains valid in the range (8.20).

To prove (8.21), we note that by sieve methods we have, for $y_{2} \geq y_{1}>0$,

$$
\Phi\left(y_{2}, z\right)-\Phi\left(y_{1}, z\right) \ll\left(y_{2}-y_{1}+z\right) V(z) .
$$

It follows that for $|v| \leq 1$,

$$
\begin{aligned}
\left|r\left(y e^{v}, z\right)-r(y, z)\right| & =\left|\frac{\Phi\left(y e^{v}, z\right)}{y e^{v} V(z)}-\frac{\Phi(y, z)}{y V(z)}\right| \\
& \leq \frac{\Phi\left(y e^{v}, z\right)}{y e^{v} V(z)}\left|1-e^{v}\right|+\frac{1}{y V(z)}\left|\Phi\left(y e^{v}, z\right)-\Phi(y, z)\right| \\
& \ll \frac{\left(y e^{v}+z\right)|v|}{y e^{v}}+\frac{y\left|e^{v}-1\right|+z}{y} \\
& \ll|v|+\frac{z}{y} .
\end{aligned}
$$

Since for any $y^{\prime}>0$,

$$
\left|r\left(y^{\prime}, z\right)\right| \leq 1+\frac{\Phi\left(y^{\prime}, z\right)}{y^{\prime} V(z)} \ll 1+\frac{z}{y^{\prime}},
$$


we obtain

$$
\begin{aligned}
r(y, z ; \lambda)= & \frac{\sqrt{\lambda}}{\sqrt{2 \pi}} \int_{-\infty}^{\infty} r\left(y e^{v}, z\right) e^{-\lambda v^{2} / 2} d v \\
= & \frac{\sqrt{\lambda}}{\sqrt{2 \pi}} \int_{-1}^{1}\left(r(y, z)+O\left(|v|+\frac{z}{y}\right)\right) e^{-\lambda v^{2} / 2} d v \\
& +O\left(\sqrt{\lambda}\left(\int_{1}^{\infty}+\int_{-\infty}^{-1}\right)\left(1+\frac{z}{y e^{v}}\right) e^{-\lambda v^{2} / 2} d v\right) \\
= & r(y, z)+O\left(\sqrt{\lambda} \int_{1}^{\infty} e^{-\lambda v^{2} / 2} d v\right)+O\left(\sqrt{\lambda} \int_{-\infty}^{\infty}|v| e^{-\lambda v^{2} / 2} d v\right) \\
& +O\left(\sqrt{\lambda} \int_{-\infty}^{\infty} \frac{z}{y e^{v}} e^{-\lambda v^{2} / 2} d v\right) \\
= & r(y, z)+O\left(e^{-\lambda / 2}\right)+O\left(\frac{1}{\sqrt{\lambda}}\right)+O\left(\frac{z}{y}\right) .
\end{aligned}
$$

This proves (8.21) and completes the proof of Theorem C.

\section{Proof of Theorem B1}

We fix a (small) positive number $\varepsilon$, real numbers $y$ and $z$ in the range $R_{\varepsilon}$, and a positive integer $q$. As before, we define $u=\log y / \log z$ and $\mathscr{L}=\log z$. Throughout this and the next section, the implied constants depend at most on $\varepsilon$, and thus, in particular, are independent of $k$.

Since the function $r_{k}$ and the hypothesis (3.5) of Theorem B1 depend only on the prime divisors of $k$ that are $<z$, we may assume that $k$ has no prime factors $\geq z$. Thus (3.5) reduces to

$$
\sum_{p \mid k} 1 \leq 2 \log \mathscr{L} .
$$

We begin with the following simple lemma:

Lemma 9.1. Let $\Sigma^{\prime}$ denote summation over integers all of whose prime factors divide $k$. We have, for any $\alpha \geq \varepsilon / 2$ and $D \geq 1$,

$$
\sum_{d \geq D}^{\prime} \frac{1}{d^{\alpha}} \ll \frac{\mathscr{L}^{1 / 2}}{D^{\alpha(1-\varepsilon / 4)}} .
$$

Proof. We have

$$
\begin{aligned}
\sum_{d \geq D}^{\prime} \frac{1}{d^{\alpha}} & \leq \sum_{d \geq 1}^{\prime} \frac{1}{d^{\alpha}}\left(\frac{d}{D}\right)^{\alpha(1-\varepsilon / 4)} \\
& =D^{-\alpha(1-\varepsilon / 4)} \sum_{d \geq 1}^{\prime} \frac{1}{d^{\alpha \varepsilon / 4}} \\
& =D^{-\alpha(1-\varepsilon / 4)} \prod_{p \mid k}\left(1-\frac{1}{p^{\alpha \varepsilon / 4}}\right)^{-1}
\end{aligned}
$$


Now if $\nu$ is the number of prime factors of $k$ and $p_{\nu}$ denotes the $\nu$ th prime, then

$$
\begin{aligned}
\prod_{p \mid k}\left(1-\frac{1}{p^{\alpha \varepsilon / 4}}\right)^{-1} & \leq \prod_{p \leq p_{\nu}}\left(1-\frac{1}{p^{\alpha \varepsilon / 4}}\right)^{-1} \leq \prod_{p \leq p_{\nu}}\left(1-\frac{1}{p^{\varepsilon^{2} / 8}}\right)^{-1} \\
& =\exp \left\{O\left(\sum_{p \leq p_{\nu}} \frac{1}{p^{\varepsilon^{2} / 8}}\right)\right\}=\exp \left\{O\left(p_{\nu}^{1-\varepsilon^{2} / 8}\right)\right\} \\
& \ll \exp \left(\nu^{1-\varepsilon^{2} / 10}\right) \ll \mathscr{L}^{1 / 2},
\end{aligned}
$$

since $p_{\nu} \ll \nu \log \nu$ and $\nu \ll \log \mathscr{L}$ by (9.1). This proves the asserted estimate.

Next, we define a smoothed form $r_{k}(y, z ; \lambda)$ of $r_{k}$, analogous to the function $r(y, z ; \lambda)$ in Theorem $\mathrm{C}$, by

$$
r_{k}(y, z ; \lambda)=\frac{\sqrt{\lambda}}{\sqrt{2 \pi}} \int_{-\infty}^{\infty} r_{k}\left(y e^{v}, z\right) e^{-\lambda v^{2} / 2} d v .
$$

The following lemma relates the functions $r_{k}(y, z ; \lambda)$ and $r(y, z ; \lambda)$. Here, and in the sequel, $\Sigma^{\prime}$ has the same meaning as in Lemma 9.1.

Lemma 9.2. For any $\lambda \geq 1$ we have

$$
r_{k}(y, z ; \lambda)=\prod_{p \mid k}\left(1-\frac{1}{p}\right)\left\{\sum_{d \leq y^{1-\varepsilon / 2}}^{\prime} \frac{1}{d} r\left(\frac{y}{d}, z ; \lambda\right)+O\left(\frac{1}{y^{1-4 \varepsilon / 5}}\right)\right\} .
$$

Proof. We start out with the identity

$$
\Phi_{k}\left(y^{\prime}, z\right)=\sum_{d \geq 1}^{\prime} \Phi\left(\frac{y^{\prime}}{d}, z\right), \quad\left(y^{\prime}>0\right),
$$

which follows easily from the definition of $\Phi_{k}(y, z)$. By Lemma 9.1 the contribution of the range $d>y^{1-\varepsilon / 2}$ to the sum is bounded by

$$
\leq \sum_{d>y^{1-\varepsilon / 2}}^{\prime} \frac{y^{\prime}}{d} \ll y^{\prime} \frac{\mathscr{L}^{1 / 2}}{y^{(1-\varepsilon / 2)(1-\varepsilon / 4)}} \ll y^{\prime} \frac{\mathscr{L}^{1 / 2}}{y^{1-3 \varepsilon / 4}}
$$

Thus we obtain

$$
\begin{aligned}
\Phi_{k}\left(y^{\prime}, z\right)= & \sum_{d \leq y^{1-\varepsilon / 2}}^{\prime} \Phi\left(\frac{y^{\prime}}{d}, z\right)+O\left(y^{\prime} \frac{\mathscr{L}^{1 / 2}}{y^{1-3 \varepsilon / 4}}\right) \\
= & \sum_{d \leq y^{1-\varepsilon / 2}}^{\prime} \frac{y^{\prime}}{d} V(z)\left(1+r\left(\frac{y^{\prime}}{d}, z\right)\right)+O\left(y^{\prime} \frac{\mathscr{L}^{1 / 2}}{y^{1-3 \varepsilon / 4}}\right) \\
= & y^{\prime} V(z) \prod_{p \mid k}\left(1-\frac{1}{p}\right)^{-1}+y^{\prime} V(z) \sum_{d \leq y^{1-\varepsilon / 2}}^{\prime} \frac{1}{d} r\left(\frac{y^{\prime}}{d}, z\right) \\
& +O\left(y^{\prime} \frac{\mathscr{L}^{1 / 2}}{y^{1-3 \varepsilon / 4}}\right),
\end{aligned}
$$


where $V(z)=\prod_{p<z}(1-1 / p)$ and, in the last estimate, we used the bound

$$
\sum_{d \leq y^{1-\varepsilon / 2}}^{\prime} \frac{y^{\prime}}{d}=y^{\prime} \sum_{d \geq 1}^{\prime} \frac{1}{d}+O\left(\sum_{d>y^{1-\varepsilon / 2}}^{\prime} \frac{y^{\prime}}{d}\right)=y^{\prime} \prod_{p \mid k}\left(1-\frac{1}{p}\right)^{-1}+O\left(y^{\prime} \frac{\mathscr{L}^{1 / 2}}{y^{1-3 \varepsilon / 4}}\right) .
$$

It follows that

$$
\begin{aligned}
r_{k}\left(y^{\prime}, z\right) & =\prod_{p \mid k}\left(1-\frac{1}{p}\right) \frac{\Phi_{k}\left(y^{\prime}, z\right)}{y^{\prime} V(z)}-1 \\
& =\prod_{p \mid k}\left(1-\frac{1}{p}\right)\left\{\sum_{d \leq y^{1-\varepsilon / 2}}^{\prime} \frac{1}{d} r\left(\frac{y^{\prime}}{d}, z\right)+O\left(\frac{\mathscr{L}^{1 / 2}}{V(z) y^{1-3 \varepsilon / 4}}\right)\right\} .
\end{aligned}
$$

Letting $y^{\prime}=y e^{v}$ and integrating both sides of this identity against the weight function $\sqrt{\lambda / 2 \pi} \exp \left(-\lambda v^{2} / 2\right)$, we obtain

$$
r_{k}(y, z ; \lambda)=\prod_{p \mid k}\left(1-\frac{1}{p}\right)\left\{\sum_{d<y^{1-\varepsilon / 2}}^{\prime} \frac{1}{d} r\left(\frac{y}{d}, z ; \lambda\right)+O\left(\frac{\mathscr{L}^{1 / 2}}{V(z) y^{1-3 \varepsilon / 4}}\right)\right\},
$$

which in view of the bound

$$
\mathscr{L}^{1 / 2} V(z)^{-1} \ll(\log z)^{3 / 2} \ll(\log y)^{3 / 2}
$$

implies the asserted estimate (9.3).

Lemma 9.3. We have

$$
\sum_{d \leq y^{1-z / 2}}^{\prime} \frac{1}{d} \rho\left(\frac{y}{d}, z\right)=c_{k}(y, z) \prod_{p \mid k}\left(1-\frac{1}{p}\right)^{-1} \rho(y, z)\left(1+O\left(\frac{1}{\mathscr{L}^{1 / 3}}\right)\right)
$$

and

$$
\sum_{d \leq y^{1-\varepsilon / 2}}^{\prime} \frac{1}{d}\left|\rho\left(\frac{y}{d}, z\right)\right| \ll c_{k}(y, z) \prod_{p \mid k}\left(1-\frac{1}{p}\right)^{-1}|\rho(y, z)|
$$

where

$$
c_{k}(y, z)=\prod_{p \mid k}\left(1-\frac{1}{p}\right)\left(1-\frac{1}{p^{1-\xi_{u} / \mathscr{L}}}\right)^{-1} .
$$

Proof. By the estimate (3.15) of Proposition 3.2 (with $\varepsilon / 2$ in place of $\varepsilon$ ), we have for $d \leq y^{1-\varepsilon / 2}$,

$$
\rho\left(\frac{y}{d}, z\right)=\rho(y, z) \exp \left\{\frac{\log d}{\mathscr{L}}\left(\xi_{u}+O(1)\right)\right\}=d^{\xi_{u} / \mathscr{L}+O(1 / \mathscr{L})} \rho(y, z) .
$$


Hence the left-hand side of (9.4) equals

$$
\begin{aligned}
& \rho(y, z) \sum_{d \leq y^{1-\varepsilon / 2}}^{\prime} \frac{1}{d^{1-\xi_{u} / \mathscr{L}+O(1 / \mathscr{L})}} \\
&=\rho(y, z)\left\{\sum_{d \geq 1}^{\prime} \frac{1}{d^{1-\xi_{u} / \mathscr{L}}}+O\left(\sum_{d \geq z}^{\prime} \frac{1}{d^{1-\xi_{u} / \mathscr{L}+O(1 / \mathscr{L})}}\right)\right. \\
&\left.\quad+O\left(\frac{1}{\mathscr{L}} \sum_{d<z}^{\prime} \frac{\log d}{d^{1-\xi_{u} / \mathscr{L}}}\right)\right\} .
\end{aligned}
$$

The main term inside the braces can be written as

$$
\sum_{d \geq 1}^{\prime} \frac{1}{d^{1-\xi_{u} / \mathscr{L}}}=\prod_{p \mid k}\left(1-\frac{1}{p^{1-\xi_{u} / \mathscr{L}}}\right)^{-1}=c_{k}(y, z) \prod_{p \mid k}\left(1-\frac{1}{p}\right)^{-1}
$$

by the definition of $c_{k}(y, z)$. Further, since $1-\xi_{u} / \mathscr{L} \geq 2 \varepsilon / 3$ in the range $R_{\varepsilon}$ (assuming that the constants $u_{0}$ and $z_{0}$ in the definition of $R_{\varepsilon}$ have been chosen sufficiently large), from Lemma 9.1 we get

$$
\sum_{d \geq z}^{\prime} \frac{1}{d^{1-\xi_{u} / \mathscr{L}+O(1 / \mathscr{L})}} \ll \sum_{d \geq z}^{\prime} \frac{1}{d^{\varepsilon / 2}} \ll \frac{\mathscr{L}^{1 / 2}}{z^{(\varepsilon / 2)(1-\varepsilon / 4)}} \ll \frac{1}{\mathscr{L}^{1 / 2}}
$$

and

$$
\begin{aligned}
\frac{1}{\mathscr{L}} \sum_{d<z}^{\prime} \frac{\log d}{d^{1-\xi_{u} / \mathscr{L}}} & \ll \frac{1}{\mathscr{L}} \sum_{p \mid k} \frac{\log p}{p^{\varepsilon / 2}} \sum_{d \geq 1}^{\prime} \frac{1}{d^{\varepsilon / 2}} \\
& \ll \frac{1}{\mathscr{L}}\left(\sum_{p \mid k} 1\right) \prod_{p \mid k}\left(1-\frac{1}{p^{\varepsilon / 2}}\right)^{-1} \ll \frac{1}{\mathscr{L}^{1 / 3}}
\end{aligned}
$$

in view of (9.1) and (9.2). Therefore, both error terms in (9.7) are bounded by $O\left(\mathscr{L}^{-1 / 3}\right)$, and the right-hand side of $(9.7)$ becomes

$$
\rho(y, z) c_{k}(y, z) \prod_{p \mid k}\left(1-\frac{1}{p}\right)^{-1}\left(1+O\left(\frac{1}{\mathscr{L}^{1 / 3}}\right)\right) .
$$

This proves (9.4); the second estimate (9.5) can be established in the same way.

For the remainder of this section we restrict $(y, z)$ as in the statement of Theorem $\mathrm{B} 1$ to the range $\left(R_{1 / 6+\varepsilon}\right)$. This restriction would not be necessary if the Riemann Hypothesis were assumed.

Lemma 9.4. There exist numbers $z_{ \pm}$in the range

$$
\min \left(z^{1-2 /(u+2)}, \frac{1}{2} z\right) \leq z_{ \pm} \leq z
$$

such that

$$
\left\{\begin{array}{l}
\operatorname{Re} \rho\left(y, z_{+}\right) \geq \exp \left\{-u \xi_{u}-c_{1} u\right\} \\
\operatorname{Re} \rho\left(y, z_{-}\right) \leq-\exp \left\{-u \xi_{u}-c_{1} u\right\}
\end{array}\right.
$$

holds, where $c_{1}$ is a suitable positive constant depending at most on $\varepsilon$. 
Proof. By Proposition 3.2 and (3.2) we have, for any $z^{\prime}$ in the range (9.8),

$$
\left|\rho\left(y, z^{\prime}\right)\right|=\exp \left\{-u^{\prime} \xi_{u^{\prime}}+O\left(u^{\prime}\right)\right\},
$$

where $u^{\prime}=\log y / \log z^{\prime}$. Since

$$
u^{\prime}=u \frac{\log z}{\log z^{\prime}}=u\left(1+O\left(\frac{1}{u}+\frac{1}{\log z}\right)\right)
$$

for $\min \left(z / 2, z^{1-2 /(u+2)}\right) \leq z^{\prime} \leq z$, we may replace $u^{\prime}$ by $u$ in this estimate. To prove (9.9), it therefore suffices to show that there exist values $z_{ \pm}$satisfying (9.8) such that

$$
\left|\operatorname{Arg} \rho\left(y, z_{+}\right)\right| \leq \pi / 3, \quad\left|\operatorname{Arg} \rho\left(y, z_{-}\right)-\pi\right| \leq \pi / 3,
$$

where Arg denotes the principal value of the argument.

First we consider the case when $u \leq \sqrt{\mathscr{L}}$. By the estimate (3.15) of Proposition 3.2 we have, for $u \leq u^{\prime} \leq u+2$,

$$
\arg \rho\left(z^{u^{\prime}}, z\right)=\arg \rho\left(z^{u}, z\right)+\pi\left(u^{\prime}-u\right)+O(1 / \log u) .
$$

Hence there exist numbers $u_{ \pm}$in $[u, u+2]$ such that

$$
\left|\operatorname{Arg} \rho\left(z^{u_{+}}, z\right)\right| \leq \pi / 4, \quad\left|\operatorname{Arg}\left(z^{u_{-}}, z\right)-\pi\right| \leq \pi / 4,
$$

provided $u \geq u_{0}$ with a sufficiently large constant $u_{0}$, as we may assume. Setting $z_{ \pm}=z^{u / u_{ \pm}}$, we then have

$$
z^{u /(u+2)} \leq z_{ \pm} \leq z
$$

so that $(9.8)$ is satisfied, and

$$
\arg \rho\left(y, z_{ \pm}\right)=\arg \rho\left(z_{ \pm}^{u_{ \pm}}, z_{ \pm}\right)=\arg \rho\left(z^{u_{ \pm}}, z\right)+O(u / \mathscr{L})
$$

by the last estimate of Proposition 3.2. In view of (9.11) and our assumption $u \leq \sqrt{\mathscr{L}}$, this implies (9.10).

This argument would go through for $u \leq z^{5 / 12}$, if we would use Huxley's prime number theorem and the estimate (3.16) of Proposition 3.1, but it would break down for larger values of $u$. For the range $\sqrt{\mathscr{L}}<u \leq z^{5 / 6-\varepsilon}$, therefore, we use a somewhat different approach that depends on an "almost all" type estimate for primes in short intervals. Let

$$
h=z \sqrt{\mathscr{L}} / u, \quad z_{n}=z-n h \quad\left(1 \leq n \leq n_{0}=[z / 2 h]\right),
$$

so that, for $1 \leq n \leq n_{0}, z / 2 \leq z_{n} \leq z$, and $z_{n}-z_{n+1}=h \gg z^{1 / 6+\varepsilon}$ by our assumption that $(y, z)$ lies in the range $\left(R_{1 / 6+\varepsilon}\right)$. We shall show that (9.9) holds with $z_{ \pm}=z_{n_{ \pm}}$for suitable indices $n_{ \pm} \leq n_{0}$.

First we note that the error term function $E(\tilde{z}, z)$ defined in Proposition 3.2 satisfies

$$
\int_{z / 2}^{z}|E(t, t+h)| d t \ll_{A} \frac{z h}{(\log z)^{A}}
$$


for any fixed constant $A$. This follows from Selberg's work [Se] and Huxley's zero-density estimate $[\mathrm{Hu}]$ in view of the bound $h \gg z^{1 / 6+\varepsilon}$; for the argument see Lemma 2 of Nicolas [Ni]. A simple consequence of this estimate is that

$$
\left|E\left(z_{n}, z_{n+1}\right)\right| \ll h / \mathscr{L}^{2}
$$

holds for all $n \leq n_{0}$ with at most $O\left(n_{0} / \mathscr{L}^{2}\right)$ exceptions. If $z$ is large enough, this implies that there exist at least $\mathscr{L}$ consecutive indices $n \leq n_{0}$ for which (9.12) holds, say $n=n_{1}+1, \ldots, n_{1}+J$, wher $J=[\mathscr{L}]$ and $1 \leq n_{1} \leq n_{0}-J$. Let

$$
u_{j}=\log y / \log z_{n_{1}+j} .
$$

By the definition of $z_{n}$ and $h$ we have, for $j=1, \ldots, J$,

$$
\begin{aligned}
u_{j+1}-u_{j} & =\frac{\log y}{\log \left(z_{n_{1}}-(j+1) h\right)}-\frac{\log y}{\log \left(z_{n_{1}}-j h\right)} \\
& =\frac{u \mathscr{L} \log \left(\left(z_{n_{1}}-j h\right) /\left(z_{n_{1}}-(j+1) h\right)\right)}{\left(\log z_{n_{1}}\right)^{2}+O(\mathscr{L})} \asymp \frac{h u}{z \mathscr{L}}=\frac{1}{\sqrt{\mathscr{L}}} .
\end{aligned}
$$

Thus, in view of (3.15), the argument of $\rho\left(z_{n_{1}}^{u_{j}}, z_{n_{1}}\right)$ increases in steps of size $\asymp 1 / \sqrt{\mathscr{L}}$, as $j$ ranges over the integers in the interval $[1, \mathscr{L}]$. It follows that, for suitable indices $j_{ \pm} \leq J$, we have (9.11) with $z_{n_{1}}$ in place of $z$ and $u_{ \pm}=u_{j_{ \pm}}$. Using the esimate (9.12) for $n=n_{1}+j, 1 \leq j \leq J$, Proposition 3.2 , and the definitions of $h$ and $J$, we see that with $z_{ \pm}=z_{n_{1}+j_{ \pm}}=y^{1 / u_{ \pm}}$, we have

$$
\begin{aligned}
\arg \rho\left(y, z_{ \pm}\right) & =\arg \rho\left(z_{n_{1}+j_{ \pm}}^{u_{ \pm}}, z_{n_{1}+j_{ \pm}}\right) \\
& =\arg \rho\left(z_{n_{1}}^{u_{ \pm}}, z_{n_{1}}\right)+O\left(\frac{u}{z} \sum_{n=n_{1}}^{n_{1}+J}\left(\left|E\left(z_{n}, z_{n+1}\right)\right|+\frac{h}{\mathscr{L}^{2}}+z^{\varepsilon}\right)\right) \\
& =\arg \rho\left(z_{n_{1}}^{u_{ \pm}}, z_{n_{1}}\right)+O\left(J \frac{u}{z}\left(\frac{h}{\mathscr{L}^{2}}+z^{\varepsilon}\right)\right) \\
& =\arg \rho\left(z_{n_{1}}^{u_{ \pm}}, z_{n_{1}}\right)+O\left(\frac{1}{\sqrt{\mathscr{L}}}\right) .
\end{aligned}
$$

This estimate, in conjunction with (9.11) (with $z_{n_{1}}$ in place of $z$ ), implies (9.10), and thus completes the proof of Lemma 9.4.

Proof of Theorem B1. From Lemma 9.2, Theorem C, and Lemma 9.3 we obtain, for $\log u \leq \lambda \leq \exp \left(\mathscr{L}^{3 / 2-\varepsilon}\right)$,

$$
\begin{aligned}
r_{k}(y, z ; \lambda)= & c_{k}(y, z)\left\{\operatorname{Re} \rho(y, z)+O\left(\frac{|\rho(y, z)|}{\log u}\right)+O\left(\frac{|\rho(y, z)|}{\mathscr{L}^{1 / 3}}\right)\right\} \\
& +O\left(\frac{1}{y^{1-4 \varepsilon / 5}}\right) .
\end{aligned}
$$


The last error term here can be estimated, using the bound $u \leq z^{5 / 6-\varepsilon}$ and Proposition 3.2, by

$$
\begin{aligned}
y^{-1+4 \varepsilon / 5} & \ll \exp \left\{-\left(1-\frac{4}{5} \varepsilon\right) u \mathscr{L}\right\} \ll \exp \left\{-\left(1+\frac{\varepsilon}{10}\right) u \xi_{u}\right\} \\
& \ll \frac{|\rho(y, z)|}{\log u} \ll c_{k}(y, z) \frac{|\rho(y, z)|}{\log u}
\end{aligned}
$$

since $c_{k}(y, z) \geq 1$. It follows that

$$
r_{k}(y, z ; \lambda)=c_{k}(y, z)\left\{\operatorname{Re} \rho(y, z)+O\left(\frac{|\rho(y, z)|}{\log u}\right)+O\left(\frac{|\rho(y, z)|}{\mathscr{L}^{1 / 3}}\right)\right\} .
$$

We apply this estimate with $y_{1}=y-y / 2 \log y$ in place of $y$, and $z_{ \pm}$in place of $z$, where the numbers $z_{ \pm}$are chosen according to Lemma 9.4 so that (9.9) (with $y$ replaced by $y_{1}$ ) is satisfied. Adjusting the constant $c_{1}$ if necessary, we obtain

$$
\pm r_{k}\left(y_{1}, z_{ \pm} ; \lambda\right) \geq \exp \left\{-u \xi_{u}-c_{1} u\right\}
$$

Note that the numbers $z_{ \pm}$here depend only on $y$ and $z$, but not on $k$ or $\lambda$.

We now set $\delta=1 / 2 \log y, \Delta=\log (1+\delta)$, so that

$$
\begin{aligned}
y & \geq y_{1}(1+\delta)=y_{1} e^{\Delta}, \\
y\left(1-\frac{1}{\log y}\right) & \leq y_{1}(1-\delta) \leq y_{1} e^{-\Delta},
\end{aligned}
$$

and choose $\lambda=(\log y)^{4}=(2 \delta)^{-4}$. With this choice of parameters we have

$$
\begin{aligned}
r_{k}\left(y_{1}, z_{+} ; \lambda\right) & =\frac{\sqrt{\lambda}}{\sqrt{2 \pi}} \int_{-\Delta}^{\Delta} r_{k}\left(y_{1} e^{v}, z_{+}\right) e^{-\lambda v^{2} / 2} d v+O\left(\sqrt{\lambda} \mathscr{L} \int_{\Delta}^{\infty} e^{-\lambda v^{2} / 2} d v\right) \\
& =\frac{\sqrt{\lambda}}{\sqrt{2 \pi}} \int_{-\Delta}^{\Delta} r_{k}\left(y_{1} e^{v}, z_{+}\right) e^{-\lambda v^{2} / 2} d v+O\left(\mathscr{L} e^{-\lambda \Delta^{2} / 2}\right) \\
& \leq \max _{|v| \leq \Delta} r_{k}\left(y_{1} e^{v}, z_{+}\right)+O(1 / y) \\
& \leq \max _{y(1-1 / \log y) \leq y^{\prime} \leq y} r_{k}\left(y^{\prime}, z_{+}\right)+O(1 / y),
\end{aligned}
$$

and a similar estimate holds with $z_{-}$in place of $z_{+}$. Combining these estimates with (9.14) yields

$$
\max _{y(1-1 / \log y) \leq y^{\prime} \leq y} \pm r_{k}\left(y^{\prime}, z_{ \pm}\right) \geq \exp \left\{-u \xi_{u}-c_{1} u\right\}+O(1 / y)
$$

In view of the hypothesis $u \leq z^{5 / 6-\varepsilon}$, the error term here is of smaller order than the main term. Therefore we obtain the desired estimates (3.7) with suitable numbers $y_{ \pm}$satisfying (3.6), after adjusting the constant $c_{1}$ if necessary.

\section{Proof of Theorem B3}

Since the proof is to a large degree analogous to that of Theorem B1, we keep the exposition short. We fix $\varepsilon>0$, real numbers $y, z$ in the range $R_{\varepsilon}$, and 
a prime $p \geq z$. We concentrate on the case when $p \geq z$; the argument for $p<z$ is similar and simpler.

We write

$$
\Phi^{(p)}(y, z)=\sum_{n \leq x}^{(p)} \frac{n}{\varphi(n)},
$$

where $\sum^{(p)}$ denotes summation over integers $n$ that are not divisible by $p$ or by any prime $<z$,

$$
V^{(p)}(z)=\prod_{p^{\prime}<z}\left(1-\frac{1}{p^{\prime}}\right)\left(1-\frac{1}{p}\right),
$$

and

$$
C=C^{(p)}(z)=\prod_{\substack{p^{\prime} \geq z \\ p^{\prime} \neq p}}\left(1+\frac{1}{p^{\prime}\left(p^{\prime}-1\right)}\right) .
$$

We recall that $r^{(p)}$ and $\bar{r}^{(p)}(y, z)$ are defined by

$$
\Phi^{(p)}(y, z)=y C V^{(p)}(z)\left(1+r^{(p)}(y, z)\right)
$$

and

$$
\bar{r}^{(p)}(y, z)=\int_{y / 2}^{y} r^{(p)}(t, z) \frac{d t}{t} .
$$

For $\lambda>0$ we set

$$
\begin{aligned}
& r^{(p)}(y, z ; \lambda)=\frac{\sqrt{\lambda}}{\sqrt{2 \pi}} \int_{-\infty}^{\infty} r^{(p)}\left(y e^{v}, z\right) e^{-\lambda v^{2} / 2} d v \\
& \bar{r}^{(p)}(y, z ; \lambda)=\int_{y / 2}^{y} r^{(p)}(t, z ; \lambda) \frac{d t}{t} .
\end{aligned}
$$

The following lemma is analogous to Lemma 9.2.

Lemma 10.1. For any $\lambda \geq 1$ we have

$$
\begin{aligned}
r^{(p)}(y, z ; \lambda)=\frac{1}{C(1-1 / p)}\{ & \sum_{d \leq y^{1-\varepsilon / 2}}^{(p)} \frac{\mu^{2}(d)}{d \varphi(d)} r\left(\frac{y}{d}, z ; \lambda\right) \\
& \left.-\frac{1}{p} \sum_{d \leq y^{1-\varepsilon / 2}}^{(p)} \frac{\mu^{2}(d)}{d \varphi(d)} r\left(\frac{y}{d p}, z ; \lambda\right)\right\}+O\left(\frac{\mathscr{L}}{y^{1-\varepsilon / 2}}\right),
\end{aligned}
$$

where the summation in $\sum^{(p)}$ is restricted to integers $d$ that are not divisible by $p$ or by any prime $<z$.

Proof. Using the identity

$$
\frac{n}{\varphi(n)}=\sum_{d \mid n} \frac{\mu^{2}(d)}{\varphi(d)} \quad(n \geq 1),
$$


we obtain, for any $y^{\prime}>0$,

$$
\begin{aligned}
& \Phi^{(p)}\left(y^{\prime}, z\right)=\sum_{n \leq y^{\prime}}^{(p)} \sum_{d \mid n} \frac{\mu^{2}(d)}{\varphi(d)}=\sum_{d \geq 1}^{(p)} \frac{\mu^{2}(d)}{\varphi(d)} \sum_{n \leq y^{\prime} / d}^{(p)} 1 \\
& =\sum_{d \geq 1}^{(p)} \frac{\mu^{2}(d)}{\varphi(d)}\left(\Phi\left(\frac{y^{\prime}}{d}, z\right)-\Phi\left(\frac{y^{\prime}}{d p}, z\right)\right) \\
& =\sum_{d \leq y^{1-\varepsilon / 2}}^{(p)} \frac{\mu^{2}(d)}{\varphi(d)} \frac{y^{\prime}}{d} V(z)\left\{1-\frac{1}{p}+r\left(\frac{y^{\prime}}{d}, z\right)-\frac{1}{p} r\left(\frac{y^{\prime}}{d p}, z\right)\right\}+O\left(\frac{y^{\prime}}{y^{1-\varepsilon / 2}}\right) \\
& =C y^{\prime} V^{(p)}(z)\left\{1+\frac{1}{C(1-1 / p)} \sum_{d \leq y^{1-\varepsilon / 2}}^{(p)} \frac{\mu^{2}(d)}{d \varphi(d)} r\left(\frac{y^{\prime}}{d}, z\right)\right. \\
& \left.\quad-\frac{1}{C(1-1 / p) p} \sum_{d \leq y^{1-\varepsilon / 2}}^{(p)} \frac{\mu^{2}(d)}{d \varphi(d)} r\left(\frac{y^{\prime}}{d p}, z\right)\right\}+O\left(\frac{y^{\prime}}{y^{1-\varepsilon / 2}}\right),
\end{aligned}
$$

where

$$
C=\sum_{d \geq 1}^{(p)} \frac{\mu^{2}(d)}{\varphi(d) d}=\prod_{\substack{p^{\prime} \geq z \\ p^{\prime} \neq p}}\left(1+\frac{1}{p^{\prime}\left(p^{\prime}-1\right)}\right)
$$

is defined as above and the error term absorbs the contribution of the range $d>y^{1-\varepsilon / 2}$ to the sum over $\Phi\left(y^{\prime} / d, z\right)$ and that of the range $d>y^{1-\varepsilon / 2}$ to the sum over $\Phi\left(y^{\prime} / d p, z\right)$. It follows that

$$
\begin{aligned}
r^{(p)}\left(y^{\prime}, z\right)=\frac{1}{C(1-1 / p)}\{ & \sum_{d \leq y^{1-\varepsilon / 2}}^{(p)} \frac{\mu^{2}(d)}{d \varphi(d)} r\left(\frac{y^{\prime}}{d}, z\right) \\
& \left.-\frac{1}{p} \sum_{d \leq y^{1-\varepsilon / 2}}^{(p)} \frac{\mu^{2}(d)}{d \varphi(d)} r\left(\frac{y^{\prime}}{d p}, z\right)\right\}+O\left(\frac{\mathscr{L}}{y^{1-\varepsilon / 2}}\right) .
\end{aligned}
$$

Integrating both sides with $y^{\prime}=y e^{v}$ against the weight function $\sqrt{\lambda / 2 \pi}$. $\cdot \exp \left(-\lambda v^{2} / 2\right)$, we obtain (10.1).

Lemma 10.2. We have

$$
\sum_{d \leq y^{1-\varepsilon / 2}}^{(p)} \frac{\mu^{2}(d)}{d \varphi(d)} \rho\left(\frac{y}{d}, z\right)=\left(1+O\left(\frac{1}{\mathscr{L}}\right)\right) \rho(y, z)
$$

and

$$
\sum_{d \leq y^{1-\varepsilon / 2}}^{(p)} \frac{\mu^{2}(d)}{d \varphi(d)}\left|\rho\left(\frac{y}{d}, z\right)\right| \ll|\rho(y, z)|
$$


Proof. This result can be proved in the same way as Lemma 9.2, using the estimates

$$
\begin{aligned}
\sum_{d \geq 1}^{(p)} \frac{\mu^{2}(d)}{d^{\sigma} \varphi(d)} & =\exp \left\{O\left(\sum_{p^{\prime} \geq z} \frac{1}{p^{\prime \sigma}\left(p^{\prime}-1\right)}\right)\right\} \\
& =1+O(1 / \sqrt{z}) \quad(\sigma \geq \varepsilon / 2)
\end{aligned}
$$

and

$$
\sum_{d \geq z}^{(p)} \frac{1}{d^{\varepsilon / 2} \varphi(d)} \ll \frac{1}{z^{\varepsilon / 4}} \ll \frac{1}{\mathscr{L}}
$$

We omit the details.

Proof of Theorem B3. From Lemma 10.1, Theorem C, Lemma 10.2, and Proposition 3.1 , we deduce that, for $\log u \leq \lambda \leq \exp \left(\mathscr{L}^{3 / 2-\varepsilon}\right)$,

$$
r^{(p)}(y, z ; \lambda)=\operatorname{Re} \rho(y, z)+O(|\rho(y, z)| / \log u) .
$$

It follows that

$$
\begin{aligned}
\bar{r}^{(p)}(y, z ; \lambda) & =\int_{y / 2}^{y} r^{(p)}\left(y^{\prime}, z ; \lambda\right) \frac{d y^{\prime}}{y^{\prime}} \\
& =\operatorname{Re} \int_{y / 2}^{y} \rho\left(y^{\prime}, z\right) \frac{d y^{\prime}}{y^{\prime}}+O\left(\frac{1}{\log u} \int_{y / 2}^{y}\left|\rho\left(y^{\prime}, z\right)\right| \frac{d y^{\prime}}{y^{\prime}}\right) .
\end{aligned}
$$

Since by Proposition 3.2,

$$
\rho\left(y^{\prime}, z\right)=\rho(y, z)\left(\frac{y^{\prime}}{y}\right)^{-\xi_{u} / \mathscr{L}}\left(1+O\left(\frac{1}{\log u}\right)\right) \quad\left(y / 2 \leq y^{\prime} \leq y\right),
$$

we have

$$
\operatorname{Re} \int_{y / 2}^{y} \rho\left(y^{\prime}, z\right) \frac{d y^{\prime}}{y^{\prime}}=\frac{2^{\xi_{u} / \mathscr{L}}-1}{\xi_{u} / \mathscr{L}} \operatorname{Re} \rho(y, z)+O\left(\frac{|\rho(y, z)|}{\log u}\right) .
$$

Replacing $y$ by $y_{1}=y-y / 2 \log y$ and applying Lemma 9.4 , we deduce that

$$
\pm \bar{r}^{(p)}\left(y_{1}, z_{ \pm} ; \lambda\right) \geq \exp \left\{-u \xi_{u}-c_{1} u\right\}
$$

holds with suitable numbers $z_{ \pm}$satisfying (3.4) and an appropriate constant $c_{1}$. Taking $\lambda=(\log y)^{4}$, we complete the proof of Theorem B3 by following the argument at the end of the preceding section.

\section{OSCILLATION THEOREMS FOR PRIMES}

\section{Proof of Theorem A1}

For the proof of Theorem A1 (as well as that of Theorem A2), we require the following result that refines Proposition 2 of [HM]. It is here that we appeal to the Linnik-Gallagher prime number theorem. 
Proposition 11.1. Fix $\varepsilon>0$. For any squarefree integer $n>1$, all of whose prime factors are $\leq n^{1-\varepsilon}$, there exists a divisor $P$ of $n$, with $n / P$ prime, such that if $(a, P)=1, x \geq P^{2}$, and $x \geq h \geq x / \exp (\sqrt{\log x})$, then

$$
\theta(x+h ; P, a)-\theta(x ; P, a)=\frac{h}{\phi(P)}\left\{1+O\left(e^{-c \log x / \log P}+e^{-c \sqrt{\log x}}\right)\right\},
$$

where $c$ is a constant depending only on $\varepsilon$.

Proof. For a given value $c>0$, a character $\chi(\bmod q)$ is called " $c$-exceptional" if $L(\beta, \chi)=0$ for some real $\beta>1-c / \log q$. It is well known (cf. [Da, p. 94]) that there exists a constant $c_{0}>0$ for which there is at most one $c_{0}$-exceptional character $\chi(\bmod q)$ for each $q \geq 2$. First we show that there is a divisor $P$ of $n$, where $n / P$ is prime, for which there are no $\varepsilon c_{0}$-exceptional characters $\chi(\bmod P)$.

Suppose that this is false and let $q_{1}$ be a divisor of $n$ with $n / q_{1}$ prime. Let $\chi_{1}$ be the $\varepsilon c_{0}$-exceptional character $\left(\bmod q_{1}\right)$ and let $p$ be a prime divisor of the conductor of $\chi_{1}$. Set $q_{2}=n / p$; note that $p$ is a divisor of $q_{1}$ and so of $n$. Let $\chi_{2}$ be the $\varepsilon c_{0}$-exceptional character $\left(\bmod q_{2}\right)$, and denote the characters of modulus $n$ induced by $\chi_{1}$ and $\chi_{2}$ as $\chi_{3}$ and $\chi_{4}$, respectively. Since $p$ divides the conductor of $\chi_{3}$ but not that of $\chi_{4}, \chi_{3}$ and $\chi_{4}$ cannot be the same character. However, since by our assumption $L\left(\beta_{i}, \chi_{i}\right)=0$ for some numbers $\beta_{i}>1-\varepsilon c_{0} / \log q_{i}$ and $i=1,2$, we have $L\left(\beta_{i}, \chi_{i+2}\right)=0$ where $\beta_{i}>1-\varepsilon c_{0} / \log q_{i} \geq 1-c_{0} / \log n$ as each $q_{i} \geq n^{\varepsilon}$. Thus $\chi_{3}$ and $\chi_{4}$ are distinct $c_{0}$-exceptional characters $(\bmod n)$. This, as was already pointed out, cannot happen.

Choosing $P$ as above we have, under the hypotheses of the proposition,

$$
\theta(x+h ; P, a)-\theta(x ; P, a)=\frac{1}{\varphi(P)} \sum_{\chi(\bmod P)} \bar{\chi}(a) \sum_{x<p \leq x+h} \chi(p) \log p
$$

and therefore,

$$
\begin{aligned}
& |\theta(x+h ; P, a)-\theta(x ; P, a)-h / \varphi(P)| \\
& \quad \leq \frac{1}{\phi(P)}\left\{\left|\sum_{x<p \leq x+h} \log p-h\right|+\sum_{\substack{k \mid P \\
k>1}} \sum_{\chi(\bmod k)}^{*}\left|\sum_{x<p \leq x+h} \chi(p) \log p\right|\right\},
\end{aligned}
$$

where in $\sum^{*}$ the summation is restricted to primitive characters. By the choice of $P$, the characters appearing in the last sum all satisfy $L(\beta, \chi) \neq 0$ for $\beta>1-\varepsilon c_{0} / \log P$. Thus, estimating the sum over $p$ by a sharp form of the prime number theorem, and the character sum by Theorem 7 of Gallagher [Ga] (with $Q=\max \{P, \exp (\sqrt{\log x})\}$ ), we obtain

$$
\left|\theta(x+h ; P, a)-\theta(x ; P, a)-\frac{h}{\varphi(P)}\right| \ll \frac{h}{\varphi(P)}\left\{e^{-c \log x / \log P}+e^{-c \sqrt{\log x}}\right\} .
$$

This proves (11.1). 
Proof of Theorem A1. We give the proof of (2.3), the proof of (2.4) being almost identical. Given $q$ and $x$ satisfying (2.1) and (2.2), we let $y=x / q$ and define $z=\log x / \log y$ so that $(y, z)$ lies in the range $\left(R_{1 / 6+\varepsilon}\right)$ of Theorem B1 (with $\left.u_{0}=1+\varepsilon\right)$ provided that $\varepsilon$ is sufficiently small and $q_{0}(\varepsilon)$ sufficiently large. We next take $z_{+}$as in Theorem B1, define $n=\prod_{p<z_{+}, p \nmid q} p$, and choose $P$ as in Proposition 11.1. Finally we set $k=q n / P$, so that

$$
P=\prod_{\substack{p<z_{+} \\ p \nmid k}} p
$$

The hypothesis (2.1) on $q$ ensures that $k$ satisfies the condition (3.5) of Theorem B1 for $q \geq q_{0}(\varepsilon)$, since $k=q n / P$ has only one prime divisor more than $q$ and we have

$$
z=\frac{\log x}{\log (x / q)} \ll \frac{\log q}{\log _{2} q}
$$

in the range (2.2). Thus by Theorem $\mathrm{B} 1$, there exists a number $y_{+}$in the range (3.6) for which the first inequality in (3.7) holds. Since

$$
\Phi_{k}\left(y_{+}, z_{+}\right)=y_{+} \prod_{\substack{p<z_{+} \\ p \nmid k}}\left(1-\frac{1}{p}\right)\left(1+r_{k}\left(y_{+}, z_{+}\right)\right)=y_{+} \frac{\varphi(P)}{P}\left(1+r_{k}\left(y_{+}, z_{+}\right)\right)
$$

by (11.2), this inequality can be restated as

$$
\Phi_{k}\left(y_{+}, z_{+}\right) \geq y_{+} \frac{\varphi(P)}{P}\left(1+\exp \left(-u \xi_{u}-c_{1} u\right)\right)
$$

where $u=\log y / \log z$.

Consider now the matrix $\mathscr{M}=\left(a_{r s}\right)$, where

$$
a_{r s}= \begin{cases}\log (r P+q s) & \text { if } r P+q s \text { prime } \\ 0 & \text { otherwise }\end{cases}
$$

and where $r$ and $s$ run over the values $r \sim R$ (that is $R<r \leq 2 R$ ) and $1 \leq s \leq y_{+}$with

$$
R=\frac{x}{2 P} \exp (-\sqrt{\log x})
$$

Let $|M|$ denote the sum of the entries of $\mathscr{M}$. For given $s$, the sum of entries in the sth column is

$$
\theta(2 R P+q s ; P, q s)-\theta(R P+q s ; P, q s) .
$$

This vanishes if $(q s, P)>1$, and for those $s$ with $(q s, P)=1$ it may be estimated by applying (11.1) with $x, h$, and $a$ replaced by $P R, P R$, and $q s$, respectively. By (11.2) and the definition of $\Phi_{k}$, there are precisely $\Phi_{k}\left(y_{+}, z_{+}\right)$ values of $s$ satisfying $(s, P)=1$, or equivalently (since $(q, P)=1),(q s, P)=$ 1. Thus, we obtain

$$
\begin{aligned}
|M| & =\frac{R P}{\varphi(P)}\left(1+O\left(e^{-c \log R P / \log P}\right)+O\left(e^{-c \sqrt{\log R P}}\right)\right) \Phi_{k}\left(y_{+}, z_{+}\right) \\
& \geq R y_{+}\left\{1+\exp \left(-u \xi_{u}-c_{1}^{\prime} u\right)\right\}
\end{aligned}
$$


with a suitable constant $c_{1}^{\prime}$, since

$$
\frac{\log R P}{\log P} \asymp \frac{\log x}{z_{+}} \geq \frac{\log x}{z}=\log y=u \log z
$$

and

$$
\sqrt{\log R P} \gg \sqrt{\log x} \geq \log y=u \log z
$$

by (11.4) and the definitions of $z_{+}$and $z$.

On the other hand, the sum of the entries in row $r$ is

$$
\theta\left(r P+q y_{+} ; q, r P\right)-\theta(r P ; q, r P)=\theta\left(r P+q y_{+} ; q, r P\right),
$$

since $r P \leq 2 R P<q$ by (11.4) and (2.2), and $r P$ is not prime. This vanishes unless $(r, q)=1$. Thus,

$$
|M|=\sum_{\substack{r \sim R \\(r, q)=1}} \theta\left(r P+q y_{+} ; q, r P\right) .
$$

The number of $r$ satisfying $r \sim R$ and $(r, q)=1$ is just $R \varphi(q) / q+O(\tau(q))$, and, since $R>x^{1 / 2}$, the error is negligible. Thus, by (11.3), (11.5), and (11.6), there is at least one $r$ for which

$$
\theta\left(r P+q y_{+} ; q, r P\right) \geq \frac{q y_{+}}{\varphi(q)}\left\{1+\exp \left(-u \xi_{u}-c_{1}^{\prime \prime} u\right)\right\}
$$

where $c_{1}^{\prime \prime}$ is an appropriate constant depending at most on $\varepsilon$. Choosing this $r$ and taking $x_{+}=r P+q y_{+}$, we obtain

$$
\Delta\left(x_{+} ; q, r P\right) \geq \exp \left(-u \xi_{u}-c_{1}^{\prime \prime} u\right)-2 r P / x_{+},
$$

which yields the desired estimate, since by (11.4) and (3.6),

$$
\begin{gathered}
x_{+}=O(x \exp (-\sqrt{\log x}))+q y(1+O(1 / \log y))=x\left(1+O\left(1 / \log _{2} x\right)\right), \\
\frac{r P}{x_{+}} \ll \exp (-\sqrt{\log x}) \ll \exp (-u \log z),
\end{gathered}
$$

and by (3.2) and the definition $\delta_{1}(x, y)$,

$$
\begin{aligned}
u \xi_{u} & =\left(1+O\left(\frac{\log _{2} u}{\log u}\right)\right) u \log u \\
& =\left(1+O\left(\frac{\log _{2} u}{\log u}\right)\right) \log y \frac{\log (\log y / \log z)}{\log z} \\
& =\left(1+O\left(\frac{\log _{2} u}{\log u}\right)\right) \delta_{1}(x, y) \log y .
\end{aligned}
$$

Remarks. Under the Generalized Riemann Hypothesis, (11.1) holds with the error replaced by $O\left(P x^{\varepsilon-1 / 2}\right)$ and, in the above proof, we may choose $z=$ $(1 / 2) \log x$ leading to the conclusion of Theorem $A 1$ with $\delta_{1}(x, y)$ replaced by

$$
\delta_{3}(x, y)=\frac{\log \left(\log y / \log _{2} x\right)}{\log _{2} x}
$$


and the larger range $q(\log q)^{1+\varepsilon} \leq x \leq q \exp \left((\log q)^{1 / 2-\varepsilon}\right)$ in place of (2.2).

As remarked in $\S 2$, we can use a modification of the above argument to delete the factor $\log _{2} x$ from (2.12) and (2.13) of Theorem A3, at least for sums of the form $\sum_{q \sim Q} \max _{(a, q)=1}, \sum_{q \sim Q} \min _{(a, q)=1}$. Indeed, since we are able to assume $\log y \ll \log _{2} x \log _{3} x$, the estimate of Corollary 3.1 , in conjunction with (2.14), is sufficient for the proof. In this case we must choose $y_{+}$above so that $q y_{+}$ is independent of $q$ and, for $y$ this small, Corollary 3.1 allows us to do that.

\section{Proof of Theorem A2}

Let $y=x / q$ and $u=\log y / \log (\log x \log y)$ so that

$$
N(\varepsilon) \ll u \leq \log ^{1 / 3} x .
$$

Define $v$ to be the positive real solution of

$$
\left[\left(2+\frac{10 v}{\log _{2} x}\right) \log x\right]^{v}=y
$$

and let $w=v / \log _{2} x$. We pick $j=[v]-1$ or $j=[v]$ so that $j$ is odd when we wish to prove (2.6) and even when we wish to prove (2.7). Clearly, if $N(\varepsilon)$ has been chosen sufficiently large, then $|j-u|<\varepsilon u / 2$.

Now take $l=y^{1 / j} / \log x, h=2 \log x$, and $z=(l+2) \log x$, so that $y=$ $(z-h)^{j}$. As

we have

$$
l=\frac{y^{1 / v}}{\log x} y^{1 / j-1 / v}=(2+10 w) \exp \left(\frac{v-j}{j v} \log y\right),
$$

$$
2+10 \frac{j}{\log _{2} x} \leq l \ll w \exp (4 / w) \ll \log ^{1 / 2} x,
$$

provided that $N(\varepsilon)$ is sufficiently large. Therefore,

$$
j^{2}(1+2 / l)^{j} \leq j^{2} e^{2 j / l} \leq(\log x)^{2 / 3}(\log x)^{1 / 5}=(\log x)^{13 / 15}
$$

as $2 j / l \leq \frac{1}{5} \log _{2} x$ by the first inequality in (12.3). Let

$$
k=1+\left[c \log x / 10 j \log _{2} x \log \left(\log _{2} x\right)\right]
$$

with $c$ as in Proposition 11.1 with $\varepsilon=1 / 2$. Then, by the last inequality in (12.3), and by (12.4),

$$
\begin{aligned}
k-j+1 & \geq \frac{c}{20} \frac{\log x}{j\left(\log _{2} x\right)^{2}}-j \\
& \geq \frac{c}{30} \frac{(\log x)^{2 / 15}}{\left(\log _{2} x\right)^{2}} j\left(1+\frac{2}{l}\right)^{j} \\
& \geq 4 j z^{j} / y
\end{aligned}
$$

so that

$$
(z-h)^{j}=y \geq \frac{4 j}{(k-j+1)} z^{j}
$$


Let $n$ be the product of any $k+1$ primes in $(z-h, z]$ that do not divide $q$. Note that, as $z^{5 / 8} \leq h \leq z$, Huxley's theorem [Hu] gives $\pi(z)-\pi(z-h) \sim$ $h / \log z$ and so there are at least $\nu(q)+k+1$ primes in $(z-h, z]$. Choose $P$ as in Proposition 11.1, with $\varepsilon=1 / 2$.

Define the matrix $\mathscr{M}$ exactly as in the proof of Theorem A1, except with $y_{+}$replaced by $y$. By counting down each column, we see that the sum of the elements of $\mathscr{M}$ is

$$
\sum_{\substack{n \leq y \\(n, \bar{P})=1}} \frac{R P}{\varphi(P)}\left\{1+O\left(e^{-c \log x / \log P}+e^{-c \sqrt{\log x}}\right)\right\}
$$

by Proposition 11.1. Now

$$
\begin{aligned}
c \frac{\log x}{\log P} & \geq \frac{c \log x}{k \log z} \geq 9 j \log \left(l \log _{2} x\right) \frac{\log _{2} x}{\log (l \log x)} \\
& \geq 2 j \log \left(j^{2} l \log _{2} x\right)
\end{aligned}
$$

by (12.3), and $c \sqrt{\log x} \geq 2 j \log \left(j^{2} l \log _{2} x\right)$. Therefore the sum of the elements of $\mathscr{M}$ is

$$
R\left(y+\frac{P}{\varphi(P)} r_{P}(y)\right)\left\{1+O\left(1 /\left(j^{2} l \log _{2} x\right)^{2 j}\right)\right\}
$$

There are

$$
R \frac{\phi(q)}{q}+O(\tau(q))=\frac{R \varphi(q)}{q}\left\{1+O\left(1 /\left(j^{2} l \log _{2} x\right)^{2 j}\right)\right\}
$$

row numbers $r$, with $r \sim R$ and $(q, r P)=1$; therefore we may choose some such row (say row $r_{0}$ ) that contains more than (respectively less than)

$$
\frac{q}{\varphi(q)}\left(y+\frac{P}{\varphi(P)} r_{P}(y)\right)\left\{1+O\left(1 /\left(j^{2} l \log _{2} x\right)^{2 j}\right)\right\}
$$

primes, in order to prove (2.6) (respectively (2.7)).

Let $x_{0}=x_{ \pm}=r_{0} P+q y$ and $a=a_{ \pm}=r_{0} P$, so that $(a, q)=1$. Now, as in the proof of Theorem A1, row $r_{0}$ contains exactly $\theta\left(x_{0} ; q, a\right)$ primes so that

$$
(-1)^{j-1} \Delta\left(x_{0} ; q, a\right) \geq(-1)^{j-1} \frac{P}{\varphi(P)} \frac{r_{P}(y)}{y}+O\left(\frac{1}{\left(j^{2} l \log _{2} x\right)^{2 j}}\right) .
$$

By Theorem B2 and (12.5) we have that

$$
\begin{aligned}
(-1)^{j-1} \frac{r_{p}(y)}{y} & \geq \frac{1}{4}\left(\begin{array}{c}
k \\
j
\end{array}\right) \frac{1}{z^{j}} \gg \frac{1}{\sqrt{j}}\left(\frac{e k}{j z}\right)^{j} \\
& \gg\left(\frac{c^{\prime}}{j^{2} l \log _{2} x \log \left(l \log _{2} x\right)}\right)^{j},
\end{aligned}
$$

where $c^{\prime}=c e / 20$, and so, by (12.7),

$$
\begin{aligned}
(-1)^{j-1} \Delta\left(x_{0} ; q, a\right) & \geq 1 /\left(u^{2} l \log _{2} x\right)^{(1+\varepsilon / 3) u} \\
& \geq 1 /\left(u^{3(1+\varepsilon / 2) u} \log ^{5} x\right)
\end{aligned}
$$

by the second inequality in (12.3). 
Finally, as $\log u=\{1+o(1)\} \log \left(\log y / \log _{2} x\right)$, we see that

$$
3(1+\varepsilon / 2) u \log u \leq(1+\varepsilon) \delta_{2}(x, y) \log y,
$$

and so (2.6) and (2.7) both follow from (12.8).

Remarks. In general this is the best possible result, using this method, up to the power of $\log x$ in the denominator. If $\log y \leq\left(\log _{2} x\right)^{2}$ then $u \leq \log _{2} x$ and so the factor $\exp (4 / w)$ in the upper bound in (12.3) becomes relevant. It does not seem possible to remove this factor because of the $l^{u}$ in the denominator of the right-hand side of (12.8). More precisely, in order to use Theorem B2, we need $l \geq 2$ and so

$$
l^{j} \log ^{j} x \geq y \geq \frac{(l+2)^{j} \log ^{j} x}{k} \geq \log ^{j-1} x .
$$

Now in the proof above we need two values of $j$, one odd and one even, so if $y=\log ^{J} x-1$ for some given integer $J$, we can take $j=J$ and $j=J-1$ only if $l \geq(\log x)^{1 /(J-1)}$. But then we are guaranteed the factor $l^{u} \approx(\log x)^{1+o(1)}$ in the denominator.

If we only need to get a good estimate on one side (i.e. for $|\Delta(x ; q, a)|)$ when $\log y$ is small compared to $\left(\log _{2} x\right)^{2}$, then we do this with $j=1+$ $[\log y / \log (2 \log x)]$ and $l=2$. Select $h, k$, and $z$ as in the proof of Theorem $\mathrm{A} 2$, and then we can get the lower bound $1 /\left(u^{2} \log _{2} x\right)^{u(1+\varepsilon)}$.

If we were to suppose that the error term in Proposition 11.1 could be taken to be $O\left(P x^{\varepsilon-1 / 2}\right)$ (which would follow from the Generalized Riemann Hypothesis) then, in the proof of Theorem A2, we could take $k=1+\left[\log x / 1000 \log _{2} x\right]$. This would imply that, in the definition of $\delta_{2}(x, y)$, we could replace the " 3 " by a " 2 " and (2.6) and (2.7) would still hold.

As remarked in $\S 2$ we can also use Theorem B2 to prove a slightly weaker version of Theorem A3 and its Corollary. By just considering those integers $q$ in $[Q, 2 Q]$ containing no prime factor in $[2 \log y, 4 \log y]$ and by taking $l=1$ in the proof of Theorem A2, one can, for example, deduce (2.15) in the range:

$$
Q \geq x \exp \left(-(1-\varepsilon) B\left(\log _{2} x\right)^{2} / \log _{3} x\right),
$$

where $A=3 B+5$.

\section{Proofs of Theorem A3 and Proposition 2.1}

We require the following simple result.

Lemma 13.1. For any nonzero integer $a$ and all $x \geq 2$, we have

$$
\begin{gathered}
\sum_{\substack{m \leq x \\
(m, a)=1}} \frac{m}{\varphi(m)}=x c_{1}(a)+O(\tau(a) \log x), \\
\sum_{\substack{m \leq x \\
(m, a)=1}} \frac{1}{\varphi(m)}=c_{1}(a) \log x+c_{2}(a)+O\left(\tau(a) x^{-1} \log x\right),
\end{gathered}
$$


where

$$
c_{1}(a)=\frac{\varphi(a)}{a} \prod_{p \nmid a}\left(1+\frac{1}{p(p-1)}\right)
$$

and $c_{2}(a) \ll \tau(a)$.

Proof. It suffices to prove (13.1) since (13.2) then follows by partial summation. Writing $m / \varphi(m)=\sum_{d \mid m} \mu^{2}(d) / \varphi(d)$ and inverting the order of summation, we have

$$
\sum_{\substack{m \leq x \\(m, a)=1}} \frac{m}{\varphi(m)}=\sum_{\substack{d \leq x \\(d, a)=1}} \frac{\mu^{2}(d)}{\varphi(d)} \sum_{\substack{r \leq x / d \\(r, a)=1}} 1
$$

Since

$$
\sum_{\substack{r \leq x / d \\(r, a)=1}} 1=\frac{x}{d} \frac{\varphi(a)}{a}+O(\tau(a))
$$

it follows that

$$
\sum_{\substack{m \leq x \\(m, a)=1}} \frac{m}{\varphi(m)}=x \frac{\varphi(a)}{a} \sum_{\substack{d \leq x \\(d, a)=1}} \frac{\mu^{2}(d)}{d \varphi(d)}+O(\tau(a) \log x)
$$

The sum over $d$ may be extended to a sum over all $d$ prime to $a$. The error in doing so is absorbed by the error $O(\tau(a) \log x)$. Once this is done, the main term is just $x c_{1}(a)$ and we have (13.1).

Proof of Theorem A3. It is well known (cf. [Da, p. 124]) that, with a suitable constant $c, 0<c<1$, the estimate

$$
\theta(x ; r, a)=x / \varphi(r)+O(x \exp (-2 c \sqrt{\log x}))
$$

holds uniformly for all $r \leq \exp (2 \sqrt{\log x})$ and all integers $a$ with $(a, r)=1$, except possibly for those moduli $r$ that are multiples of a certain exceptional modulus $r_{1}>1$. It follows that if $a$ is a nonzero integer satisfying $\left(a, r_{1}\right)>1$ (if $r_{1}$ exists), then uniformly for $R_{1} \leq R_{2} \leq \exp (c \sqrt{\log x}), x^{1 / 2} \leq x_{1} \leq x_{2} \leq$ $x$, we have

$$
\sum_{\substack{R_{1}<r \leq R_{2} \\(r, a)=1}}\left\{\theta\left(x_{2} ; r, a\right)-\theta\left(x_{1} ; r, a\right)\right\}=\sum_{\substack{R_{1}<r \leq R_{2} \\(r, a)=1}} \frac{x_{2}-x_{1}}{\varphi(r)}+O\left(x \exp \left(-c^{\prime} \sqrt{\log x}\right)\right)
$$

for any $c^{\prime}<2$. Now let $x \exp (-c \sqrt{\log x}) \leq Q_{1} \leq x / 2$ and $0<|a|<Q_{1}$. 
Then

$$
\begin{aligned}
\sum_{\substack{q \sim Q_{1} \\
(q, a)=1}} \theta(x ; q, a)= & \sum_{\substack{q \sim Q_{1} \\
(q, a)=1}} \sum_{\substack{a<p \leq x \\
p-a=q r}} \log p+O\left(Q_{1} \log |a|\right) \\
= & \sum_{\substack{r \leq x / 2 Q_{1} \\
(r, a)=1}}\left\{\theta\left(a+2 r Q_{1} ; r, a\right)-\theta\left(a+r Q_{1} ; r, a\right)\right\} \\
& +\sum_{\substack{r \sim x / 2 Q_{1} \\
(r, a)=1}}\left\{\theta(x ; r, a)-\theta\left(a+r Q_{1} ; r, a\right)\right\} \\
& +O\left(Q_{1} \log |a|\right)+O\left(Q_{1} \log _{2}\left(2 x / Q_{1}\right)\right)
\end{aligned}
$$

where the first error term absorbs the contribution of the primes $\leq a$ to $\theta(x ; q, a)$ and thus, in view of the bound $|a|<Q_{1}<q$, may be dropped unless $a$ itself is a prime. The latter error term accounts for the replacement of $(x-a)$ by $x$ in the ranges of summation. By (13.3) this implies

$$
\begin{aligned}
\sum_{\substack{q \sim Q_{1} \\
(q, a)=1}} \theta(x ; q, a)= & \sum_{\substack{r \leq x / 2 Q_{1} \\
(r, a)=1}} \frac{r Q_{1}}{\varphi(r)}-\sum_{\substack{r \sim x / 2 Q_{1} \\
(r, a)=1}} \frac{r Q_{1}}{\varphi(r)} \\
& +\sum_{\substack{r \sim x / 2 Q_{1} \\
(r, a)=1}} \frac{x}{\varphi(r)}+O\left(Q_{1} \log \left(|a| x / Q_{1}\right)\right),
\end{aligned}
$$

provided that $\left(a, r_{1}\right)>1$ in the case that the exceptional modulus $r_{1}$ exists. Setting

$$
S_{a}(t)=\sum_{\substack{r \leq t \\(r, a)=1}} r / \varphi(r)
$$

we can write the right-hand side as

$$
\begin{array}{r}
Q_{1} S_{a}\left(\frac{x}{2 Q_{1}}\right)-Q_{1}\left(S_{a}\left(\frac{x}{Q_{1}}\right)-S_{a}\left(\frac{x}{2 Q_{1}}\right)\right) \\
+x \sum_{\substack{r \sim x / 2 Q_{1} \\
(r, a)=1}} \frac{1}{\varphi(r)}+O\left(Q_{1} \log \left(|a| x / Q_{1}\right)\right)
\end{array}
$$

which, by partial summation, reduces to

$$
x \int_{x / 2 Q_{1}}^{x / Q_{1}} S_{a}(t) \frac{d t}{t^{2}}+O\left(Q_{1} \log \left(|a| x / Q_{1}\right)\right) \text {. }
$$

Since

$$
\begin{aligned}
\sum_{\substack{q \sim Q_{1} \\
(q, a)=1}} \theta(x ; q, a) & =x\left\{\sum_{\substack{q \sim Q_{1} \\
(q, a)=1}} \frac{1}{\varphi(q)}+\sum_{\substack{q \sim Q_{1} \\
(q, a)=1}} \frac{1}{\varphi(q)} \Delta(x ; q, a)\right\} \\
& =x\left\{c_{1}(a) \log 2+\sum_{\substack{q \sim Q_{1} \\
(q, a)=1}} \frac{1}{\varphi(q)} \Delta(x ; q, a)\right\}+O\left(x^{1 / 2}\right)
\end{aligned}
$$


by Lemma 13.1 and the bound $\tau(a) \ll|a|^{1 / 3} \leq Q_{1}^{1 / 3} \leq x^{1 / 3}$, it follows that (13.4)

$$
\sum_{\substack{q \sim Q_{1} \\(q, a)=1}} \frac{1}{\varphi(q)} \Delta(x ; q, a)=\int_{x / 2 Q_{1}}^{x / Q_{1}}\left(S_{a}(t)-t c_{1}(a)\right) \frac{d t}{t^{2}}+O\left(\frac{Q_{1} \log \left(|a| x / Q_{1}\right)}{x}\right) .
$$

Now, let $x$ and $Q$ be given subject to (2.11) and let $y=x / Q$. We give the proof of (2.13), the proof of (2.12) being almost identical. Set $z=(1 / 2) \log x$. In case there is an exceptional modulus $r_{1} \leq \exp (\sqrt{\log x})$, let $p$ be a prime divisor of $r_{1}$, otherwise let $p=2$. By Theorem B3 there exist $z_{-}$in the range (3.4) and $y_{-}$in the range (3.6) so that $\bar{r}^{p}\left(y_{-}, z_{-}\right)$satisfies (3.7). Let $Q_{-}=x / y_{-}$ and $a_{-}=p \prod_{p^{\prime}<z_{-}} p^{\prime}$. By (3.6) $Q_{-}$satisfies $Q \leq Q_{-} \leq Q(1-1 / \log y)^{-1}$, and thus, in view of (2.11), lies in the range $x \exp (-c \sqrt{\log x}) \leq Q_{-} \leq x / 2$. Moreover, we have $a_{-} \neq 0, Q_{-}>\left|a_{-}\right|$, and, if $r_{1}$ exists, $\left(a_{-}, r_{1}\right) \geq\left(a_{-}, p\right)=$ $p>1$. Hence the above argument applies with $Q_{1}=Q_{-}, a=a_{-}$, and (13.4) holds. Now it is easily seen that, with the above choice of $a_{-}$,

$$
\int_{x / 2 Q_{-}}^{x / Q_{-}}\left(S_{a-}(t)-t c_{1}\left(a_{-}\right)\right) \frac{d t}{t^{2}}=c_{1}\left(a_{-}\right) \bar{r}^{p}\left(y_{-}, z_{-}\right) .
$$

Thus, (2.13) follows from (13.4), (3.7), and the estimate

$$
c_{1}\left(a_{-}\right) \gg \frac{\varphi\left(a_{-}\right)}{a_{-}} \gg \prod_{p^{\prime}<z_{-}}\left(1-\frac{1}{p^{\prime}}\right) \gg \frac{1}{\log z_{-}} \gg \frac{1}{\log _{2} x} \text {. }
$$

Proof of Proposition 2.1. In the case $Q \leq x^{2 / 3}$, the result follows at once from Theorem 9 of [BFI] (for fixed $a$ ) or from Fouvry [Fo]; in fact, their results apply in a much larger range.

In the case $Q>x^{2 / 3}$, we follow the above argument except that instead of using the estimate (13.3) we bound $\theta(x ; r, a)$ on average by means of the Bombieri-Vinogradov theorem. In this way, we obtain (13.4) with $Q_{1}=Q$ and an additional error term $O_{A}\left((\log x)^{-A}\right)$ on the right-hand side. Since by (13.1),

$$
\int_{x / 2 Q}^{x / Q}\left(S_{a}(t)-t c_{1}(a)\right) \frac{d t}{t^{2}}=O\left(\int_{x / 2 Q}^{x / Q} \tau(a) \log t \frac{d t}{t^{2}}\right)=O\left(\tau(a) \frac{Q \log (x / Q)}{x}\right),
$$

this proves the desired estimate (2.16).

\section{REFERENCES}

[BFI] E. Bombieri, J. B. Friedlander, and H. Iwaniec, Primes in arithmetic progressions to large moduli, Acta Math. 156 (1986), 203-251; II Math. Ann. 277 (1987), 361-393; III J. Amer. Math. Soc. 2 (1989), 215-224.

[Bu] A. A. Buchstab, On an asymptotic estimate of the number of numbers of an arithmetic progression which are not divisible by relatively small prime numbers, Mat. Sb. (N.S.) 28 (1951), 165-184. (Russian) 
[CG] A. Y. Cheer and D. A. Goldston, $A$ differential delay equation arising from the sieve of Eratosthenes, Math. Comp. 55 (1990), 129-161.

[Da] H. Davenport, Multiplicative number theory, 2nd ed., Springer-Verlag, New York, 1980.

[E1] P. D. T. A. Elliott, Some applications of a theorem of Raikov to number theory, J. Number Theory 2 (1970), 22-55.

[E2] _ Probabilistic number theory, Vol. I, Springer-Verlag, New York, 1979.

[EH] P. D. T. A. Elliott and H. Halberstam, A conjecture in prime number theory, Sympos. Math. IV Rome (1968-69), 59-72.

[FG] J. Friedlander and A. Granville, Limitations to the equi-distribution of primes I, Ann. Math. 129 (1989), 363-382.

[Fo] E. Fouvry, Sur le problème des diviseurs de Titchmarch, J. Riene Angew. Math. 357 (1985), 51-76.

[Ga] P. X. Gallagher, A large sieve density estimate near $\sigma=1$, Invent. Math. 11 (1970), 329339.

[HaT] R. R. Hall and G. Tenenbaum, Divisors, Cambridge Tracts in Math., vol. 90, 1988.

[HM] A. Hildebrand and H. Maier, Irregularities in the distribution of primes in short intervals, J. Reine Angew. Math. 397 (1989), 162-193.

[HT] A. Hildebrand and G. Tenenbaum, On integers free of large prime factors, Trans. Amer. Math. Soc. 296 (1986), 265-290.

[Hu] M. Huxley, On the difference between consecutive primes, Invent. Math. 15 (1972), 164-170.

[Iv] A. Ivic, The Riemann zeta-function, Wiley, New York, 1985.

[Iw] H. Iwaniec, Rosser's sieve-bilinear forms of the remainder terms-some applications, Recent Progr. in Analytic Number Theory, Vol. I (H. Halberstam and C. Hooley, eds.), Academic Press, London, 1982, pp. 203-230.

[Ma] H. Maier, Primes in short intervals, Michigan Math. J. 32 (1985), 221-225.

[Mo] H. L. Montgomery, Problems concerning prime numbers, Proc. Sympos. Pure Math., vol. 28, Amer. Math. Soc., Providence, RI, 1976, pp. 307-310.

[Ni] J. L. Nicolas, Répartition des nombres largements composés, Acta Arith. 34 (1979), 379-390.

[Se] A. Selberg, On the normal density of primes in small intervals and the difference between consecutive primes, Arch. Math. Naturvid. 47 (1943), 87-105.

(J. Friedlander, A. Granville) Department of Mathematics, University of Toronto, Toronto, Ontario M5S 1A1, Canada

Department of Mathematics, University of Illinois, Urbana, Illinois 61801

Department of Mathematics, University of Georgia, Athens, Georgia 30602 